\title{
Cation and Anion Transport in a Dicationic Imidazolium-Based Plastic Crystal Ion Conductor
}

\author{
Bryce E. Kidd \\ Thesis submitted to the faculty of the \\ Virginia Polytechnic Institute and State University \\ in partial fulfillment of the requirements for the degree \\ Master of Science \\ in \\ Chemistry \\ Louis A. Madsen, Chair \\ John R. Morris \\ Harry W. Gibson \\ May $9^{\text {th }}, 2013$ \\ Blacksburg, Virginia
}

Keywords: organic ionic plastic crystal, grain boundary, NMR, self-diffusion, activation energy, Stokes-Einstein relation. 


\title{
Cation and Anion Transport in a Dicationic Imidazolium-Based Plastic Crystal Ion Conductor
}

\author{
Bryce E. Kidd
}

\begin{abstract}
Here we investigate the organic ionic plastic crystal (OIPC) 1,2-bis[ $\left[N-\left(N^{\prime}-\right.\right.$ hexylimidazolium- $\left.\left.d_{2}(4,5)\right)\right] \mathrm{C}_{2} \mathrm{H}_{4} 2 \mathrm{PF}_{6}^{-}$in one of its solid plastic crystal phases by means of multi-nuclear solid-state (SS) NMR and pulsed-field-gradient (PFG) NMR. We quantify distinct cation and anion diffusion coefficients as well as the diffusion activation energies $\left(E_{\mathrm{a}}\right)$ in this dicationic imidazolium-based OIPC. Our studies suggest a change in transport mechanism for the cation upon varying thermal and magnetic treatment $(9.4 \mathrm{~T})$, evidenced by changes in cation and anion $E_{\mathrm{a}}$. Moreover, variable temperature ${ }^{2} \mathrm{H}$ SSNMR lineshapes further support a change in local molecular environment upon slow cooling in $B_{0}$. Additionally, we quantify the percentage of mobile anions as a function of temperature from variable temperature ${ }^{19} \mathrm{~F}$ SSNMR, where two distinct spectral features are present. We also comment on the pre-exponential factor $\left(D_{0}\right)$, giving insight into the number of degrees of freedom for both cation and anion as a function of thermal treatment. In conjunction with previously reported conductivity values for this class of OIPCs and the Stokes-Einstein relation, we propose that ion conduction is dominated by anion diffusion between crystallites (i.e., grain boundaries). Using our experimentally determine diffusion coefficient and previously reported $\mathrm{PF}_{6}^{-}$hydrodynamic radius $\left(r_{H}\right)$, viscous $(\eta=4.1 \mathrm{~Pa} \cdot \mathrm{s})$ ionic liquid (IL) is present with a cation $r_{H}$ of $0.34 \mathrm{~nm}$. NMR measurements are very powerful in elucidating fundamental OIPC properties and allow a deeper understanding of ion transport within such materials.
\end{abstract}




\section{Acknowledgements}

First and foremost, this work would not have been possible without the immeasurable support from Dr. Louis Madsen. Thank you for your encouraging words, correcting me when correction was needed, guiding me, and being an overall friend. You have been the most powerful professional educator that has made an impression on me. Your teaching, discipline, morals, and values are what I consider to be the foundation for being a successful educator and scientist.

Of the educators that I have had the opportunity to learn from, the ones that I admire the most are my parents, Brenda and Brent Kidd. You have dealt with the ups and downs only a stubborn son could bring, and yet you still have stood by my side; I can never thank you enough. Moreover, this Thesis would also have not been possible without the inspiration of my grandfather, John Natale. You have given me so much to grow up to and I will always strive to be like you. For all the times I felt down, I look at the Monsanto pocket protector you gave me, "Let Safety Shine in 89 ," to lift my spirits. You will always be remembered and hold a special place in my heart 'big guy.' Spencer, words cannot describe the joy, completeness, and love you have brought me. I cannot thank you enough and I look forward to the many years we will spend together.

I would finally like to thank my remaining Thesis committee, Dr. John Morris and Dr. Harry Gibson. From you both I have learned that it is perseverance, the will to fail and get back up, that makes a successful scientist. You had confidence in my ability to be successful; thank you. 


\section{Attribution}

Throughout this research project, multiple peers have guided my work. Chapter 3 summarizes the results and will soon be submitted for publication. A brief description of their contributions towards this publication has been added here.

Chapter 3: Cation and Anion Transport in a Dicationic Imidazolium-based Plastic Ion Conductor

Chapter 3 will be submitted to The Journal of Physical Chemistry B.

Mark D. Lingwood, PhD (Dr. Louis Madsen's Lab, Department of Chemistry) is currently an assistant professor in chemistry at Saint Mary's College of California. Dr. Lingwood was a coauthor on this paper and aided in data analysis and edits.

Minjae Lee, PhD (Dr. Harry Gibson's Lab, Department of Chemistry) is currently an assistant professor in chemistry at Kunsan University in South Korea. Dr. Lee was a co-author on this paper and synthesized the material used in this study and provided edits.

Harry W. Gibson, $\mathrm{PhD}$ (Faculty Member, Department of Chemistry) is currently a professor in chemistry at Virginia Tech. Dr. Gibson served as a committee member for this Thesis and committee chair for Dr. Lee. Dr. Gibson was a co-author on this paper and provided deep discussions on understanding the data.

Louis A. Madsen, $\mathrm{PhD}$ (Faculty Member, Department of Chemistry) is currently an associate professor in chemistry at Virginia Tech. Dr. Madsen served as the committee chair for this Thesis and provided deep discussions on understanding the data. 


\section{Table of Contents}

Acknowledgements

Attribution

List of Figures

\section{Chapter 1: Organic Ionic Plastic Crystals}

1.1 General Motivations and Project Goals

1.2 Introduction to OIPCs

1.3 How Defects Influence Ion Mobility

1.4 Molecular Diffusion and $E_{\mathrm{a}}$

1.5 Joint Rotational and Translational Motion

1.6 Thermal History of OIPCs

1.7 Concluding Remarks

References

Chapter 2: Nuclear Magnetic Resonance Theory and Methods

2.1 Basic Nuclear Magnetic Resonance (NMR) Theory 42

2.2 General Remarks on Solid-State NMR $\quad 43$

2.2.1 ${ }^{2} \mathrm{H}$ NMR Theory $\quad 44$

2.3 Pulsed-Field-Gradient NMR 49

References $\quad 54$

Chapter 3: Cation and Anion Transport in a Dicationic Imidazolium-based Plastic Crystal Ion Conductor

Abstract

3.1 Introduction $\quad 58$

$\begin{array}{ll}3.2 \text { Experimental } & 62\end{array}$

3.2.1 Sample Preparation $\quad 62$

3.2.2 Variable Temperature Solid-State NMR 63

3.2.3 Variable Temperature Pulsed-Field-Gradient NMR 64

3.3 Results and Discussion $\quad 64$

3.3.1 ${ }^{2} \mathrm{H}$ NMR vs. Temperature $\quad 65$

3.3.2 ${ }^{19} \mathrm{~F}$ and ${ }^{1} \mathrm{H}$ NMR vs. Temperature $\quad 69$

$\begin{array}{ll}\text { 3.3.3 Thermal Treatments } & 76\end{array}$

3.3.4 Diffusion Coefficient vs. Temperature $\quad 77$

$\begin{array}{ll}3.4 \text { Conclusions } & 83\end{array}$

Acknowledgements $\quad 85$

$\begin{array}{ll}\text { References } & 85\end{array}$ 


\section{List of Figures}

\section{Chapter 1}

Figure 1.1 Structure of an OIPC (top: 1,2-bis $\left[N-\left(N\right.\right.$ '-hexylimidazolium- $\left.\left.d_{2}(4,5)\right)\right] \mathrm{C}_{2} \mathrm{H}_{4}$ hexafluorophosphate) and an IL (bottom: 1-butyl-3-methylimidazolium tetrafluoroborate).........5

Figure 1.2 DSC thermogram for 1,2-bis $\left[N-\left(N^{\prime}\right.\right.$-hexylimidazolium- $\left.\left.d_{2}(4,5)\right)\right] \mathrm{C}_{2} \mathrm{H}_{4} 2 \mathrm{PF}_{6}^{-}$displaying

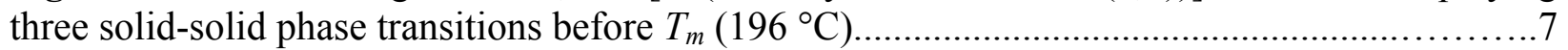

Figure 1.3 Illustration of Schottky disorder. Schottky disorder is defined as a cation-anion pair missing from the crystallite, thus conserving charge neutrality...........................

Figure 1.4 DSC thermogram of $\left[\mathrm{P}_{11} \mathrm{TFSA}\right]$ displaying three solid-solid phase transitions prior to

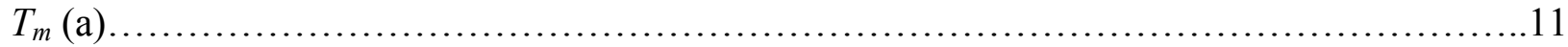

Figure 1.5 A polycrystalline material forms from the formation of nuclei in the liquid state (a),

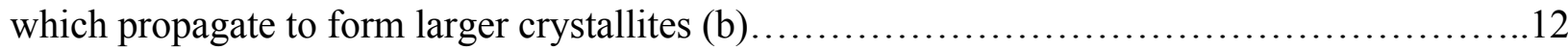

Figure 1.6 Increasing the temperature of a polycrystalline material containing grain boundaries (a) promotes the formation a larger grain boundary density (b) through the coalescence of vacancies. .13

Figure 1.7 Stacked ${ }^{1} \mathrm{H}$ NMR spectra as a function of temperature for the molecular plastic crystal 1,4-dioxane. At $0{ }^{\circ} \mathrm{C}$ (a), a narrow component on top of a broad component is observed

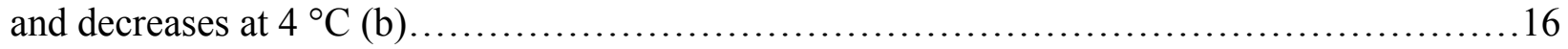

Figure 1.8 DSC thermogram (a), conductivity inset (a), and ${ }^{19} \mathrm{~F}$ variable temperature SSNMR

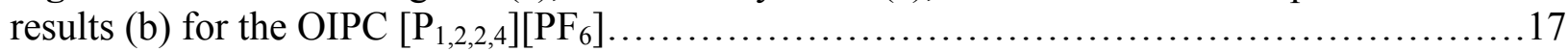

Figure 1.9 Stacked ${ }^{7} \mathrm{Li}$ NMR spectra as a function of temperature for the OIPC $\left[\mathrm{P}_{12} \mathrm{TFSI}\right]$ doped with $9.3 \mathrm{~mol} \% \mathrm{Li}^{+}$(b), $\mathrm{Li}^{+}$mobility is observed by the narrowing of the NMR signal (a) (i.e.,

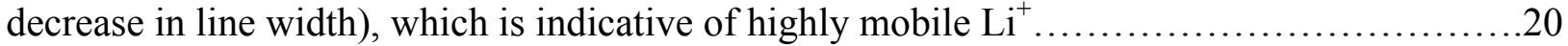

Figure 1.10 Stacked ${ }^{1} \mathrm{H}$ NMR spectra as a function of temperature for the pure OIPC (a),

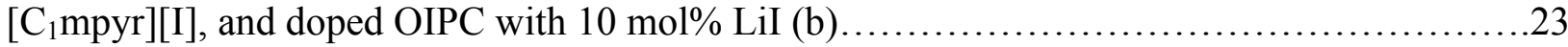

Figure 1.11 Illustration of the Brownian motion a particle experiences due to the thermal "kicks" the particle receives from neighboring particles.

Figure 1.12 Expansion of Figure 1.3 allows us to illustrate an ionic compound, such as $\mathrm{Li}_{2} \mathrm{SO}_{4}$, where the cation (blue) "hops" from one lattice position to another past the anions (yellow/red)

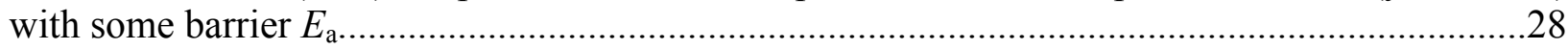

Figure 1.13 Illustration of the paddle-wheel mechanism where the revolving molecule is $\mathrm{SO}_{4}{ }^{2-}$ and the small translating ion is $\mathrm{Li}^{+}$..... 
Figure 1.14 Expansion of Figure 1.12 illustrates that upon rotation of the anion (yellow/red), the barrier to translation for the cation (blue) decreases...................................................................34

Figure 1.15 Illustration of the crystal structure for 1,2-bis[N-(N'-butylimidazolium$\left.\left.d_{2}(4,5)\right)\right] \mathrm{C}_{2} \mathrm{H}_{4} 2 \mathrm{PF}_{6}^{-}$viewed along the $b$-axis (a) and $b^{*}$-axis (b), showing the ease of rotational mobility for $\mathrm{PF}_{6}^{-}$ 36

Figure 1.16 Variable temperature ${ }^{1} \mathrm{H}$ NMR spectra for the "as-prepared" and annealed OIPC [Choline][DHP] .38

\section{Chapter 2}

Figure 2.1 In the presence of an externally applied magnetic field, the nuclear spin states of NMR active nuclei are no longer degenerate and thus split, commonly referred to as Zeeman splitting.......

Figure 2.2 Illustration of $\Delta v_{Q}$ as a function of $\theta$ for an isotropic material........ 46

Figure 2.3 A distribution of first-order splittings produces the well known Pake doublet; where most of the signal intensity comes from the $\mathrm{C}-{ }^{2} \mathrm{H}$ bonds perpendicular, $\theta=90^{\circ}$, to the magnetic field. 47

Figure 2.4 The quadrupolar spin-echo pulse sequence is commonly used in solid materials to refocus ${ }^{2} \mathrm{H}$ magnetization that relaxes upon excitation of the $90_{\mathrm{y}}{ }^{\circ}$ pulse.

Figure 2.5 Illustration of isotropic motional averaging occurring above $T_{m}$ .48

Figure 2.6 A simple spin echo pulse sequence in the presence of a pair of matched externally applied magnetic field gradients is commonly used to determine the diffusion coefficient by linearly varying the strength of an externally applied magnetic field gradient. .51

Figure 2.7 A gaussian decay in signal intensity $I$ as a function of gradient strength $g$ is observed when carrying out the PFG-NMR experiment.

\section{Chapter 3}

Figure 3.1 Chemical structure and phase map of the ionic compound 1,2-bis $\left[N-\left(N^{\prime}-\right.\right.$ hexylimidazolium- $\left.\left.d_{2}(4,5)\right)\right] \mathrm{C}_{2} \mathrm{H}_{4} 2 \mathrm{PF}_{6}^{-}$used in this study.

Figure 3.2 Room temperature $\left(25^{\circ} \mathrm{C}\right){ }^{2} \mathrm{H}$ NMR measurement of the OIPC 1,2-bis[ $N-(N$ 'hexylimidazolium- $\left.\left.d_{2}(4,5)\right)\right] \mathrm{C}_{2} \mathrm{H}_{4} 2 \mathrm{PF}_{6}{ }^{-}$displays the well-known Pake powder pattern, confirming the random orientation of crystallites with respect to $B_{0}$ .66

Figure 3.3 ${ }^{2} \mathrm{H}$ NMR spectra vs. temperature displaying imidazolium orientation and dynamics in phases $\mathrm{I}, \mathrm{S}_{1}$, and $\mathrm{S}_{3}$

Figure 3.4 Illustration of potential crystallite realignment in the presence of a magnetic field upon heating to phase I and cooling to phase $\mathrm{S}_{3}$.... 
Figure $3.5{ }^{19} \mathrm{~F}$ spectra as a function of temperature. Spectra at $205{ }^{\circ} \mathrm{C}, 200{ }^{\circ} \mathrm{C}$, and $180{ }^{\circ} \mathrm{C}$ are decreased in vertical scale by a factor of 10, 10, and 4, respectively, relative to the other

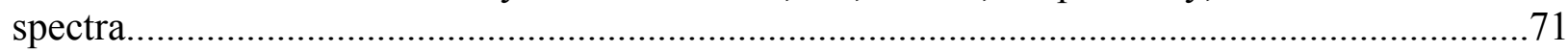

Figure 3.6 Percentage of mobile $\mathrm{PF}_{6}^{-}$(e.g., in the grain boundary) as a function of

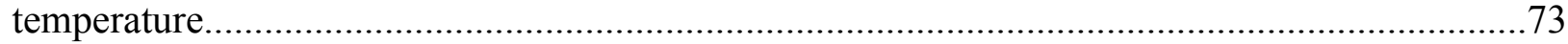

Figure $3.7{ }^{1} \mathrm{H}$ NMR spectra as a function of temperature................................................... 75

Figure 3.8 Comparison of static and $T_{2}$-weighted $\mathrm{PFG}{ }^{1} \mathrm{H}$ (a) and ${ }^{19} \mathrm{~F}$ (b) spectra, all within phase $\mathrm{S}_{3}$, emphasizing that our PFG-NMR measurements are measuring mobile cations and anions

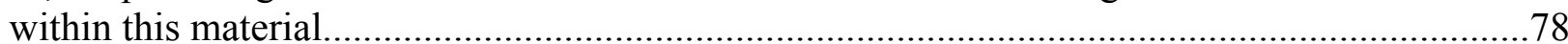

Figure 3.9 Temperature dependence of diffusion coefficient for both cation and anion as a function of thermal treatment and summary of all $E_{\mathrm{a}}$ values extracted from Arrhenius plots......79

Figure 3.10 Cation and anion $D_{0}$ values as a function of thermal treatment. Cation $D_{0}$ decreases by almost four orders of magnitude from fast cooling out of $B_{0}$ (disordered environment) to slow cooling in $B_{0}$ (more rigid environment). 


\section{Chapter 1: Organic Ionic Plastic Crystals}

\subsection{General Motivations and Project Goals}

The demand for new green technology grows each day, as evidenced by extensive research to produce more earth-friendly products, such as new batteries and the introduction of fuel cells into everyday applications where such technologies are desired (i.e., small electronic devices and possibly automobiles). While the battery and fuel cell date back to the 1800 's, ${ }^{1}$ advances in fundamental chemistry and material design have afforded new technology. The fuel cell consists of a pair of electrodes, one anode and one cathode, and an electrolyte separating the two. A fuel source, such as $\mathrm{H}_{2}$, is oxidized at the anode, where $\mathrm{H}^{+}$and electrons are generated. To conserve charge neutrality of the system, $\mathrm{H}^{+}$diffuses through the electrolyte to combine with $\mathrm{O}_{2}$ (from ambient air) and electrons (from the external circuit) through a reduction at the cathode to produce the byproduct, $\mathrm{H}_{2} \mathrm{O}$. Thus chemical energy can be converted to electrical energy. One of the main issues in implementing a novel battery or fuel cell is finding an electrolyte that enables sufficient ion transport from anode to cathode. Small ions, such as $\mathrm{H}^{+}$and $\mathrm{Li}^{+}$, generally allow the fastest transport of such ions from the anode to the cathode. More specifically, the transport of such ions is largely dependent on the electrolyte. Much research has gone into the development of solid-state electrolytes, as these materials provide a static matrix for the transport of small ions and the promise of a "greener" tomorrow.

In the past decades, polymer electrolyte membranes (PEMs), such as Nafion ${ }^{\circledR}$ (Scheme $1){ }^{2}$ have garnered much interest as a new solid-state electrolyte in fuel cell and battery technology applications due to their highly desirable small ion transport properties. ${ }^{3,4}$ 


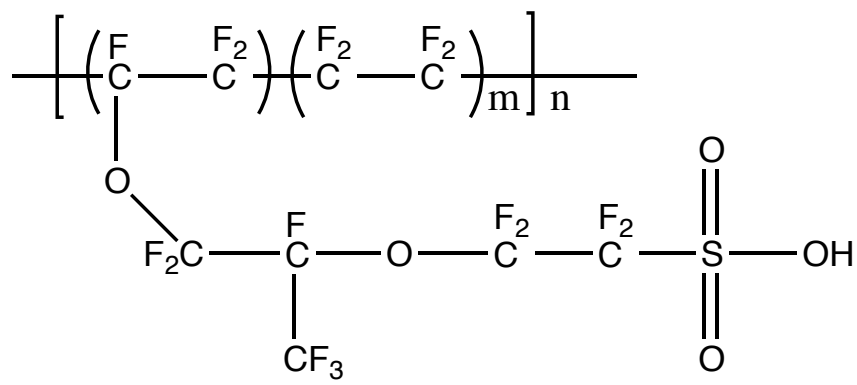

Scheme 1: Chemical structure of Nafion ${ }^{\circledR 2}$.

Nafion ${ }^{\circledR}$ is a tetrafluoroethylene copolymer functionalized with sulfonic acid terminated perfluorovinyl ether along the polymer backbone. ${ }^{2}$ The hydrophobic and hydrophilic portions nanophase separate to produce a distinct morphology consisting of ion channels lined with sulfonic acid groups $;^{2}$ thus transport of small ions, such as $\mathrm{H}^{+}, \mathrm{Li}^{+}$, and $\mathrm{Na}^{+}$, and small charged molecules, such as ionic liquids (ILs), ${ }^{5}$ is possible. Ion transport inside PEMs is highly dependent on electrostatic interactions between the mobile ions and the polymer network (i.e., $\left.\mathrm{SO}_{3}{ }^{-}\right){ }^{6}$ which can restrict ion motion and potentially reduce conductivity, thus being a drawback. Another drawback to PEMs is that operating temperatures no greater than $100{ }^{\circ} \mathrm{C}$ must be used due to the volatility of the hydrated electrolyte, thus leading to dehydration of the PEM and ultimately to a reduction in conductivity. ${ }^{7}$

Another class of solid-state electrolytes, ceramic conductors, such as $\alpha-\mathrm{Li}_{2} \mathrm{SO}_{4}(\mathrm{Scheme}$ $2),{ }^{8}$ is also known for its highly conductive properties.

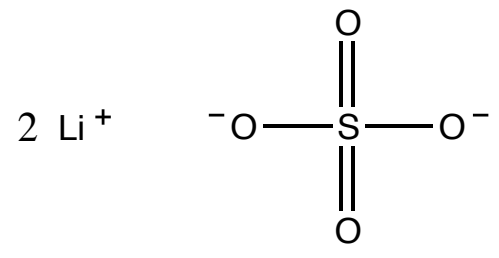

Scheme 2: Chemical structure of $\alpha-\mathrm{Li}_{2} \mathrm{SO}_{4}{ }^{8}$ 
Ceramic conductors are typically inorganic salts, in which the conduction of the native small cation is partially due to the short-range disorder present below the melt temperature $\left(T_{m}\right)$ of the material. More specifically, the short-range disorder is attributed to the "paddle-wheel" rotational mechanism associated with the cation/anion. ${ }^{9}$ Within $\alpha-\mathrm{Li}_{2} \mathrm{SO}_{4}$, sulfate acts as a "swinging door" in the crystalline lattice (i.e., isotropic rotation about one or more axes) such that the barrier to translation for lithium decreases upon the rotation of sulfate. Thus remarkably high ion conduction $\left(10^{-3} \mathrm{~S} \mathrm{~cm}^{-1}\right)$ at elevated temperatures $\left(575^{\circ} \mathrm{C}\right)$ is possible. ${ }^{8}$ The joint rotational and translational mechanism will be discussed in section 1.5.1. Unfortunately, ceramic conductors are brittle materials; thus they suffer from poor mechanical properties, limiting potential applications.

In contrast, organic ionic plastic crystals (OIPCs) represent a new and interesting form of solid-state electrolyte. ${ }^{10,11}$ After the introduction of OIPCs in the early 1990s, much research went into optimizing OIPCs for electrochemical devices and dye-sensitized solar cells. ${ }^{10}$ OIPCs are known to have multiple solid phases in which ion transport is potentially enhanced in the "rotator phase." doping of the salt form of small ions, such as lithium; ${ }^{12-14}$ in which the native cations and anions within the crystalline lattice act as a medium for the lithium to conduct, resulting in enhanced conductivity by several orders of magnitude. ${ }^{15}$ The doping of small ions into OIPCs will be discussed in more detail in section 1.3.1. While these materials display markedly high conductivity values at ambient temperatures, little is known about the diffusive nature of the cation and anion. Fundamental studies on the ion transport properties within OIPCs are necessary to fully understand the conduction process. High conductivity, low volatility, non-flammability, 
and high thermal and electrochemical stability make small ion-doped OIPCs attractive candidates as new solid-state electrolytes for fuel cell and battery applications.

Recently, Lee et al. have reported the first dicationic imidazolium-based OIPC with two imidazoliums tethered by an ethylene linker, thus promoting efficient packing of the cations and anions. $^{16,17}$ Multiple first-order solid-solid phase transitions were reported from differential scanning calorimetry (DSC) measurements with $T_{m}$ 's in the $200{ }^{\circ} \mathrm{C}$ range, which are dependent on the counterion, such as tetrafluoroborate $\left[\mathrm{BF}_{4}^{-}\right]$and hexafluorophosphate $\left[\mathrm{PF}_{6}^{-}\right]$, and the many hydrogen-fluorine contacts, reported from single crystal X-ray diffraction. ${ }^{17}$ While conductivities on the order of $10^{-5}$ to $10^{-14} \mathrm{~S} \mathrm{~cm}^{-1}$ make these pure materials impractical for future applications, ${ }^{16}$ fundamental research can help elucidate temperature-dependent phase behavior and deepen the current understanding of conduction mechanisms within OIPCs.

The purpose of the present research project is to develop an understanding of ion transport within OIPCs. Conductivity values on the order of $10^{-3} \mathrm{~S} \mathrm{~cm}^{-1}$ have been reported for novel OIPCs, thus indicating a promising future in battery and fuel cell technology applications. First, a review of OIPCs will be conducted followed by a discussion of fundamental questions and concepts, such as:

- What are the mechanisms for ion transport in OIPCs?

- What is the translational barrier $\left(E_{a}\right)$ to mobility for the cation and anion in the solid phase (i.e., potential rotator phase) below $T_{m}$ ?

- Is it possible to control the OIPC grain domain alignment in the presence of a strong magnetic field?

Nuclear magnetic resonance (NMR) is a powerful tool to answer such questions. Nuclear/chemical specificity and dynamics on multiple time scales are only two of the many 
advantages that NMR offers in comparison to other analytical techniques, such as DSC and impedance spectroscopy, and will be considered and discussed below. In the present work, I aim to answer the above questions with the OIPC 1,2-bis[ $N$ - $\left(N^{\prime}\right.$-hexylimidazolium- $\left.\left.d_{2}(4,5)\right)\right] \mathrm{C}_{2} \mathrm{H}_{4}$ hexafluorophosphate), while gaining an understanding of ion transport in such systems.

\subsection{Introduction to OIPCs}
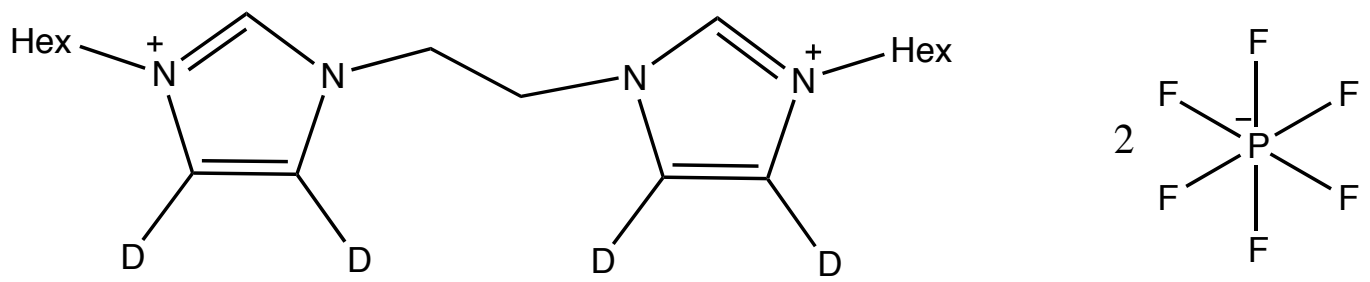

1,2-bis $\left[N\right.$ - $\left(N\right.$ '-hexylimidazolium- $\left.\left.d_{2}(4,5)\right)\right] \mathrm{C}_{2} \mathrm{H}_{4} 2 \mathrm{PF}_{6}-$
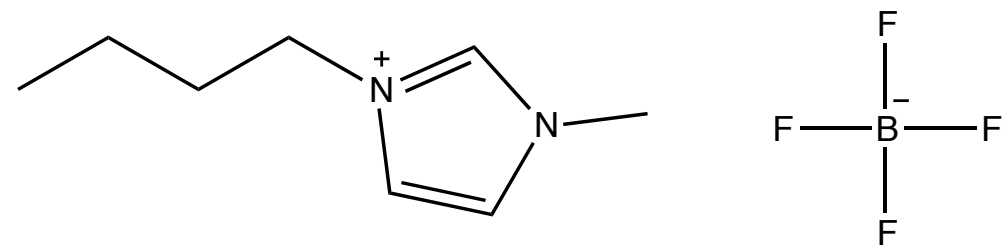

$[\mathrm{bmim}]\left[\mathrm{BF}_{4}\right]$

Figure 1.1: Structure of an OIPC (top: 1,2-bis $\left[N-\left(N^{\prime}\right.\right.$-hexylimidazolium- $\left.\left.d_{2}(4,5)\right)\right] \mathrm{C}_{2} \mathrm{H}_{4}$ hexafluorophosphate) ${ }^{16}$ and an IL (bottom: 1-butyl-3-methylimidazolium tetrafluoroborate). ${ }^{18}$ Chemically similar to ILs, OIPCs are solid at room temperature $\left(25^{\circ} \mathrm{C}\right)$ while an IL is a liquid salt.

OIPCs resemble ionic liquids (IL) in the sense that they each consist of a cation and anion (Figure 1.1). ${ }^{11,16-18}$ At room temperature $\left(25^{\circ} \mathrm{C}\right)$, an ionic liquid is a liquid salt, while an OIPC is a solid salt. ${ }^{11}$ Unlike an ionic liquid, OIPCs exhibit efficient packing through long-range crystalline order of ions, and short-range disorder where isotropic states (i.e., isotropic rotational 
mobility of the cation and/or anion about one or more axes) can exist below the melting temperature. ${ }^{11,16,17,19-31}$ During the 1960 's, Timmermans showed that molecular compounds in the solid state, such as cyclohexane (which shows such rotational mobility at $-87^{\circ} \mathrm{C}$ before melting at $\left.6{ }^{\circ} \mathrm{C}\right),{ }^{19}$ rotate freely because their shape is "quasi-spherical" upon rotation, with the centers of mass occupying fixed sites within the crystalline lattice. ${ }^{19}$ The term "rotator phase" has been coined for the rotational motions in the crystalline lattice, which are reached through one or more highly enthalpic first-order solid-solid phase transitions. ${ }^{10}$ A first-order phase transition is defined as a discontinuous change in density as a function of temperature. ${ }^{32}$ For OIPCs, a volume expansion of the crystallites at a solid-solid phase transition allows more degrees of freedom to be available, thus increasing entropy. ${ }^{33}$ Rotator phases of OIPCs contribute to the short-range disorder accompanied by low entropy of fusion; $\Delta S_{f}<20 \mathrm{~J} \mathrm{~K}^{-1} \mathrm{~mol}^{-}$ ${ }^{1}$, and is commonly known as Timmermans criterion. ${ }^{11,19}$ Pringle and MacFarlane et al. have suggested that Timmermans criterion may only be satisfied for systems that contain only one molecular species. ${ }^{11}$ However, OIPCs contain more than one molecular species, which are ionically bound. ${ }^{11}$ If the material consists of a cation-anion pair, the entropy of fusion may potentially be higher due to only one of the molecular ions exhibiting internal degrees of freedom below $T_{m}$, hence a larger $\Delta S_{f}{ }^{11}$ While Timmermans criterion has been used extensively to sift through the many OIPCs studied thus far, it is also apparent that other fundamental properties need to be considered as well. Certain OIPCs have shown high conductivities upon entering a new solid phase, but molecular interactions, molecular reorientations, and ion transport within such phases still remain a mystery. ${ }^{11}$ 


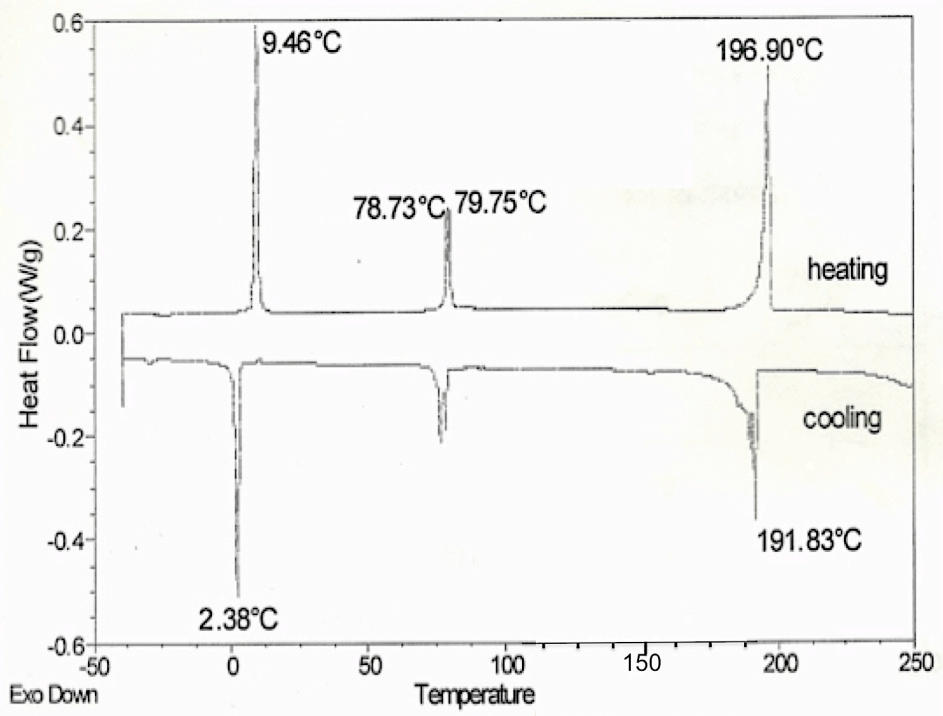

Figure 1.2: DSC thermogram for 1,2-bis $\left[N-\left(N^{\prime}\right.\right.$-hexylimidazolium- $\left.\left.d_{2}(4,5)\right)\right] \mathrm{C}_{2} \mathrm{H}_{4} \quad 2 \mathrm{PF}_{6}^{-}$ displaying three solid-solid phase transitions before $T_{m}\left(196^{\circ} \mathrm{C}\right)$. Figure reproduced from Lee $e t$ al. ${ }^{17}$

OIPCs have been studied extensively through DSC, where it has been shown that many classes of OIPCs posses multiple first-order high enthalpy solid-solid phase transitions before $T_{m}$ (Figure 1.2). ${ }^{10,11,16,17}$ DSC measurements can be complimented with impedance spectroscopic measurements (i.e., ionic conductivity) to better understand conductive properties in the solid phases of OIPCs. By comparing DSC thermograms to impedance spectroscopic measurements, it has also been shown that an increase in conductivity upon heating is observed at a solid-solid phase transition. ${ }^{11}$ Conductivity values (represented as $\sigma$ with units of $\mathrm{S} \mathrm{cm}^{-1}$, where Siemens is commonly used to represent the electrical current a material can conduct) on the order of $10^{-3} \mathrm{~S}$ $\mathrm{cm}^{-1} 10,11,31$ have been reported for OIPCs, naturally raising the question, why is there an increase in conductivity as a function of temperature? To increase conductivity as a function of temperature, the diffusivity and concentration of the charge carrying species must increase as well. Molecular diffusion within OIPCs is possible due to the nature of defects present, such as 
rotator phases, lattice vacancies, and grain boundaries. ${ }^{33-35}$ Generally for an OIPC, the higher the conductivity at ambient temperatures (i.e., below $100^{\circ} \mathrm{C}$ ), the better the material will perform in applications. However, to be able to design a highly conductive material, one must fully understand the fundamental interactions that influence the translational motions that in turn give rise to conductivity. OIPCs have become of great interest in the past decades due to conductivity enhancements evidenced in one or more solid phases, but little knowledge has been gained on how cation and/or anion transport occurs. There are a large number of cation-anion pairs and currently it is impossible to predict which pair will show the best performance. It is imperative to understand the fundamental inter- and intramolecular interactions that give rise to ion transport to rationally develop OIPCs for future applications.

\subsection{How Defects Influence Ion Mobility}

With the ultimate goal of understanding the mechanism for ion transport in OIPCs, we must first start with how ion transport in any ionic solid occurs. For ion transport to occur within an ionic solid, defects (in the form of lattice vacancies) must be present. ${ }^{36}$ One type of lattice

disorder that is commonly present in ionic solids is Schottky disorder, ${ }^{36,37}$ as seen in Figure $1.3 .^{12}$ 


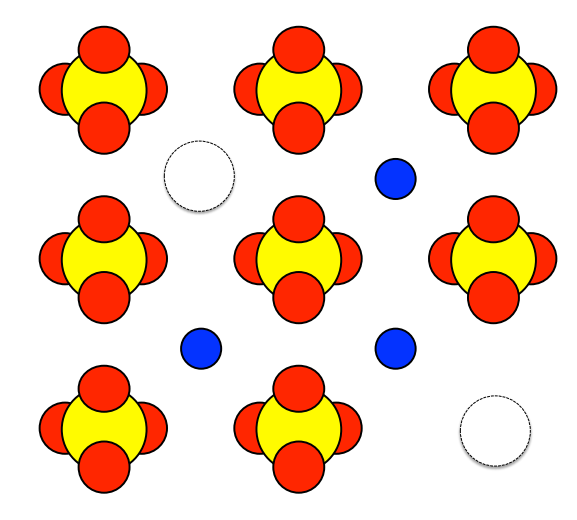

Schottky Disorder

Figure 1.3: Illustration of Schottky disorder. Schottky disorder is defined as a cation-anion pair missing from the crystallite, thus conserving charge neutrality. ${ }^{36}$ Figure adapted from MacFarlane et al. ${ }^{12}$

Schottky disorder is classified as a cation-anion pair missing from the crystalline lattice and thus contributes to the overall short-range disorder present in an ionic material. ${ }^{36}$ With each ion missing, the counterion will be missing as well to conserve charge neutrality of the crystallite. ${ }^{37}$ Schottky disorder occurs due to the second law of thermodynamics, whereby entropy drives the formation of defects. ${ }^{36}$ Vacancies in an ionic solid can facilitate translational motion of ions, which can strongly impact ion conduction. Other relevant factors, such as vacancy size, critical volume (minimal volume needed to allow translational and/or rotational mobility of the ion), ${ }^{38}$ and ion size also influence ion transport, which are all dependent on the cation-anion pair. In the presence of Schottky disorder, ion transport can occur through an "ion hopping" mechanism. For ion hopping to occur, there must be an open vacancy within the lattice for the diffusing species to occupy; hence ion transport is influenced by the presence and size of lattice vacancies. ${ }^{36}$ However, to be able to increase diffusion along with conductivity in ionic solids, the diffusive species must carry the charge (i.e., single charged species may diffuse through small vacancies, 
thus increasing conductivity, while a cation/anion pair may diffuse through larger vacancies, but this would not increase conductivity). ${ }^{33}$

Positron annihilation lifetime spectroscopy (PALS) can be used to quantify the size and number of vacancies for ionic solids. PALS studies are conducted by injecting the sample with positrons (typically from a ${ }^{22} \mathrm{Na}^{+}$source) to form a positron-electron bound state, also known as positronium. The lifetime of a positron is dependent on whether it exists in a highly dense electron area or not, hence a positron that exists in a vacancy and does not annihilate (i.e., the electron and positron do not collide to produce $\gamma$ photons) will have a longer life-time when compared to a positron not within a vacancy. ${ }^{36}$ Huang et al. conducted studies using PALS and showed that the conduction of the OIPC N,N-dimethylpyrrolidinium bis(trifluoromethanesulfonyl)imide, $\left[\mathrm{P}_{11} \mathrm{TFSI}\right]$ (Scheme 3), is highly dependent on the size of the crystalline vacancies. $^{33}$
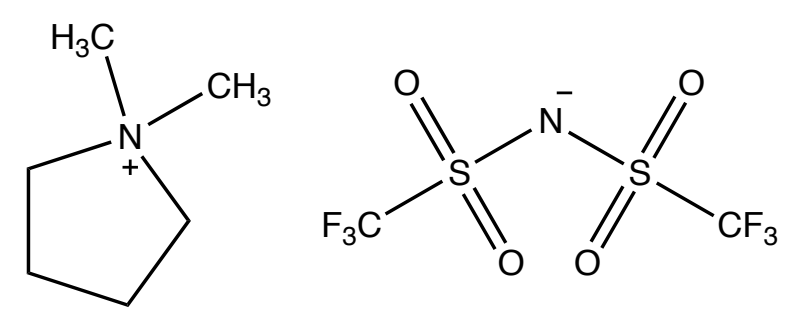

Scheme 3: Chemical structure of $\left[\mathrm{P}_{11} \mathrm{TFSI}\right]{ }^{33}$

Their studies were focused on the third to highest phase transition (phase III to II), as phase II showed the highest conductivity value for the material (Figure 1.4a). Figure 1.4b, a CohenTurnbull free volume conduction plot, shows the dependency of conductivity $\sigma$ on vacancy volume $V_{f}$ for $\left[\mathrm{P}_{11} \mathrm{TFSA}\right]$, where a change in slope (i.e., change in the critical volume of a vacancy) is observed at the phase III to II transition. ${ }^{33}$ 


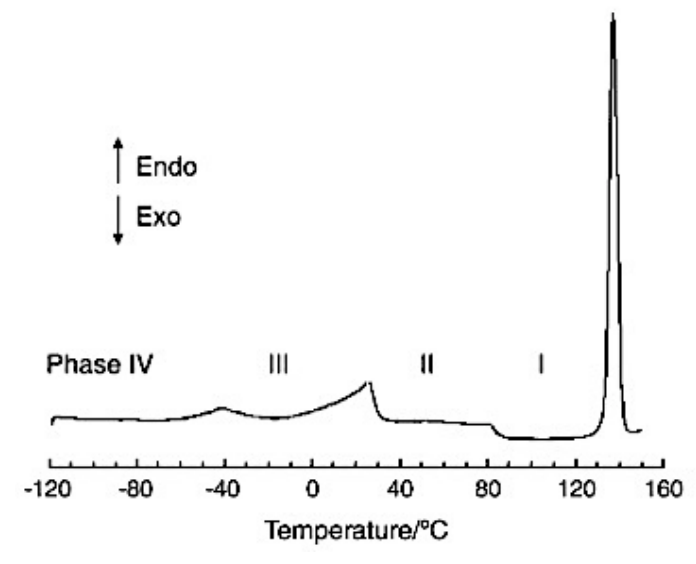

(a)

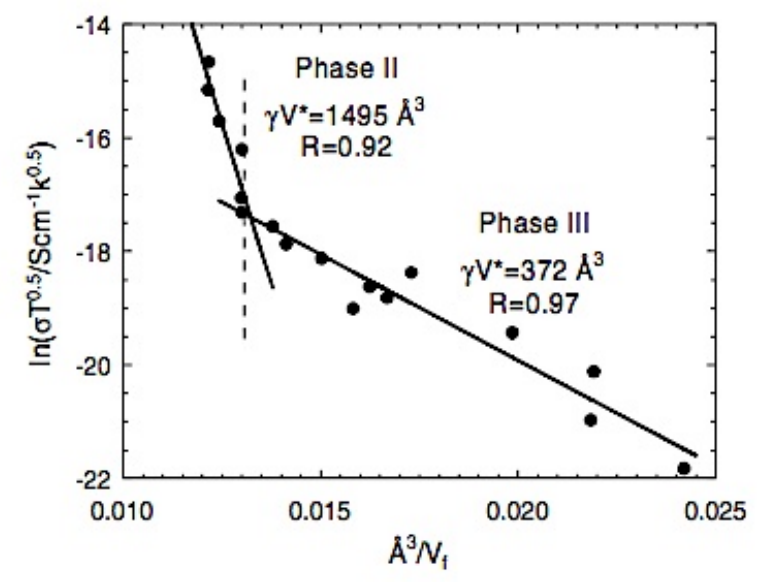

(b)

Figure 1.4: DSC thermogram of $\left[\mathrm{P}_{11}\right.$ TFSA] displaying three solid-solid phase transitions prior to $T_{m}$ (a). A Cohen-Turnbull plot relates conductivity to vacancy volume where a discontinuous change in slope is suggestive of a change in diffusion mechanism, as observed at the phase III $\left(V_{f}\right.$ $\left.=372 \AA^{3}\right)$ to II $\left(V_{f}=1495 \AA^{3}\right)$ transition temperature for [P $\mathrm{P}_{11} \mathrm{TFSI}$ (b). Figures reproduced from Huang et al. ${ }^{33}$ Used under fair use, 2013.

The change in vacancy volume from phase III to II, 372 to $1495 \AA^{3}$, respectively, was suggested to be due to a change in the diffusion mechanism for the ions. ${ }^{33}$ The unit cell volume for $\left[\mathrm{P}_{11} \mathrm{TFSI}\right]$ was previously reported prior to Huang's studies and found to be $1488 \AA^{3}$, with a cation-anion pair volume of $\sim 372 \AA^{3},{ }^{39}$ which is in good agreement with the change in slope observed by Huang et al. It was concluded that in phase III the vacancy volume is large enough to support a cation-anion pair, suggestive of Schottky disorder with conduction of ions attributed to ion hopping through the crystalline lattice. ${ }^{33}$ Interestingly, in phase II, the vacancy volume is much larger than a cation or anion vacancy and a cation-anion divacancy, suggestive of an extended network of defects (i.e., grain boundary), which will be discussed below. 
Extending our view from ion transport on the sub-nanometer scale to ion transport on the nanometer to micron-scale in a polycrystalline material, another possible ion transport pathway is through grain boundaries. ${ }^{36}$ Since OIPCs are polycrystalline materials, it has also been suggested that ion transport can occur via grain boundaries, ${ }^{10}$ which are highly dependent on the cation-anion pair. The grain boundary naturally occurs for polycrystalline materials and originates from the mismatch of crystallites upon nucleation from the melt (Figure 1.5a,b, and c) and through the coalescence of vacancies upon heating (Figure $1.6 \mathrm{a}$ and $\mathrm{b}$ ). ${ }^{36}$ In the literature, the grain boundary is sometimes referred to as an extended network of vacancies/defects. ${ }^{29}$

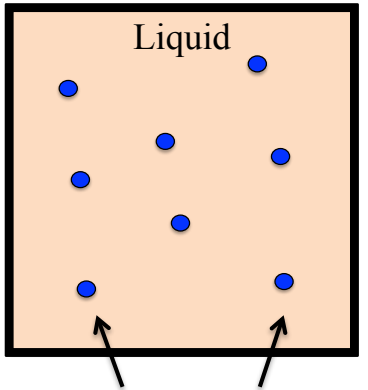

Nuclei

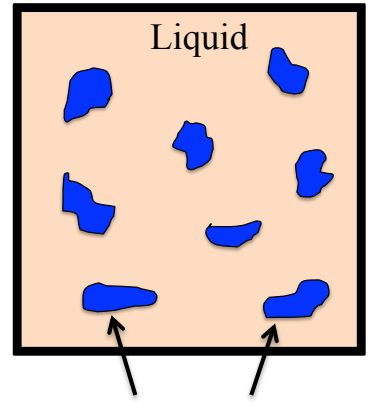

Crystallite Formation

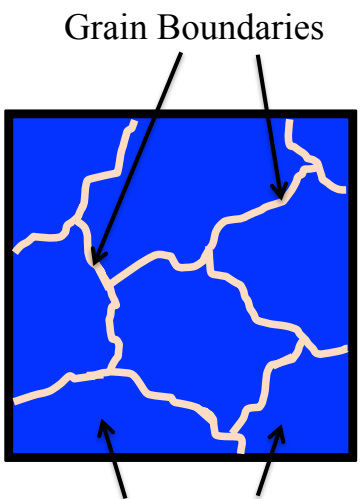

Crystallites

(a)

(c)

Figure 1.5: A polycrystalline material forms from the formation of nuclei in the liquid state (a), which propagate to form larger crystallites (b). Finally, due to the mismatch of crystallites upon formation, nanometer to micron-scale grain boundaries form (c). 


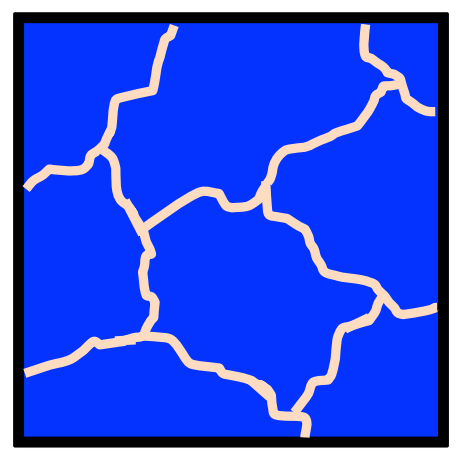

(a)

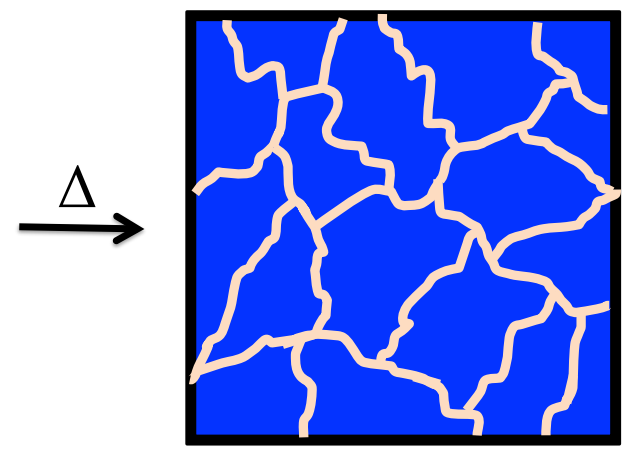

(b)

Figure 1.6: Increasing the temperature of a polycrystalline material containing grain boundaries (a) promotes the formation a larger grain boundary density (b) through the coalescence of vacancies. The coalescence of vacancies can be thought of as an extended network of defects on the nanometer to micron-scale. ${ }^{29}$

Considering a polycrystalline material riddled with Schottky-type defects, a segregation of mobile ions will occur upon heating to form smaller crystallites and a higher density of grain boundaries while conserving charge neutrality globally. The formation of smaller crystallites results in a larger grain boundary density. ${ }^{29}$ Grain boundary formation is also temperature dependent, and like conductivity, could occur in a gradual or step-wise manner at a solid-solid phase transition. Recall, Huang et al. observed a volume expansion of the crystalline lattice for $\left[\mathrm{P}_{11}\right.$ TFSI $]$ at the phase III to II transition. ${ }^{33}$ A first-order phase transition can promote the coalescence of multiple vacancies, potentially forming grain boundaries. An increase in the number of pathways for diffusion (i.e., increase in the number of degrees of freedom for the ions) can lead to an increase in ionic conductivity. Such a change in diffusive behavior upon vacancy volume expansion for a similar OIPC, $\left[\mathrm{P}_{12} \mathrm{TFSI}\right]$ (Scheme 4), was previously reported by Pas et al. $^{35}$ 

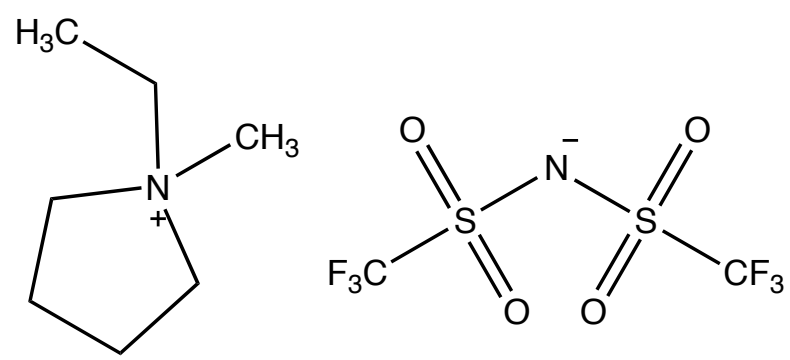

Scheme 4: Chemical structure of $\left[\mathrm{P}_{12} \mathrm{TFSI}\right] .^{35}$

Interestingly, grain boundaries act as a nucleation point for the melt phase. Close to $T_{m}$ of an OIPC, the liquid phase can potentially form at the grain boundary before the remaining material melts (commonly known as the "premelting phenomenon"). ${ }^{40}$ Dosseh et al. used variable temperature ${ }^{1} \mathrm{H}$ solid-state nuclear magnetic resonance (SSNMR) to study the phase behavior for the molecular plastic crystals 1,4-dioxane $\left(T_{m}=11{ }^{\circ} \mathrm{C}\right)($ Scheme 5), cyclohexanol $\left(T_{m}=25^{\circ} \mathrm{C}\right)$, cyclohexane $\left(T_{m}=7^{\circ} \mathrm{C}\right)$, pivalic acid $\left(T_{m}=37^{\circ} \mathrm{C}\right)$, and succinonitrile $\left(T_{m}=58\right.$ $\left.{ }^{\circ} \mathrm{C}\right) .{ }^{40}$ Specifically, Dosseh et al. were interested in the phase behavior of these materials below $T_{m}$ due to observed liquid-phase formation at the grain boundary within the temperature range studied. Figure 1.7 is stacked ${ }^{1} \mathrm{H}$ NMR spectra for 1,4 -dioxane as a function of temperature, displaying two distinct spectral features (i.e., one narrow signal and one broad signal).

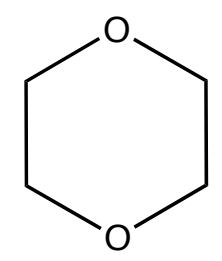

Scheme 5: Chemical structure of 1,4-dioxane. ${ }^{40}$ 
A narrow signal on top of a broad signal can clearly be seen at $0{ }^{\circ} \mathrm{C}$ (Figure $1.7 \mathrm{a}$ ). Interestingly, upon heating to $8{ }^{\circ} \mathrm{C}$ (Figure $1.7 \mathrm{c}$ ), the majority of the broad signal diminishes, leaving behind the narrow signal. ${ }^{40}$ One explanation to these observed spectral features is a system composed of a crystalline and amorphous component, wherein the crystalline component represents a rigid environment and non-isotropic averaging of the molecules (producing a broad signal) and the amorphous component potentially allows isotropic tumbling (producing a narrow signal). As temperature approaches $T_{m}$, melting at the surface of the crystallites begins, thus increasing the population of mobile ions in the grain boundary; hence the broad component will decrease as a function of temperature. Dosseh et al. concluded that the narrow component was 1,4-dioxane in the grain boundary and the broad component was 1,4-dioxane in the crystalline lattice. ${ }^{40}$ 

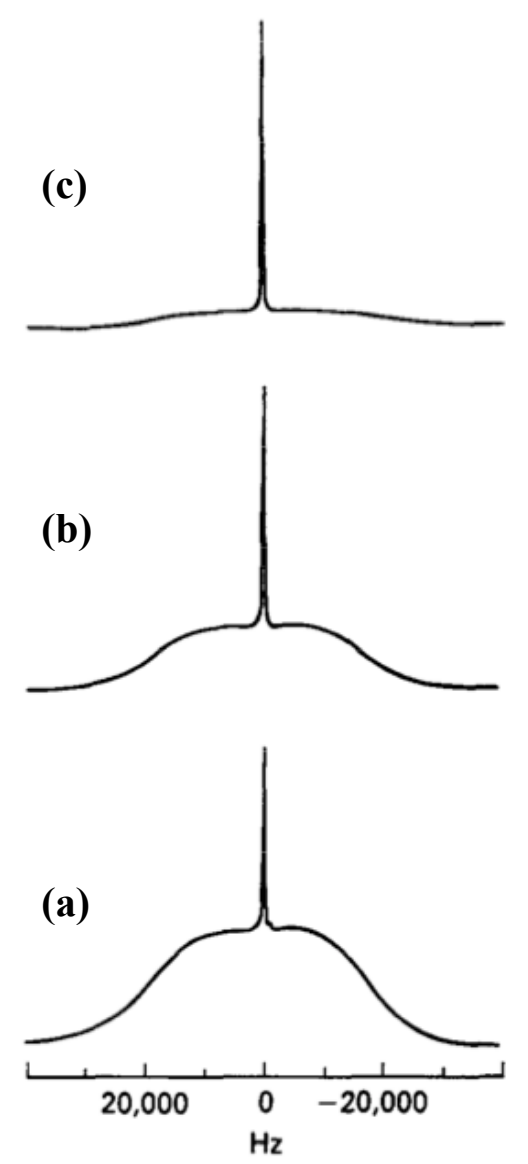

Figure 1.7: Stacked ${ }^{1} \mathrm{H}$ NMR spectra as a function of temperature for the molecular plastic crystal 1,4 -dioxane. At $0{ }^{\circ} \mathrm{C}(\mathrm{a})$, a narrow component on top of a broad component is observed and decreases at $4{ }^{\circ} \mathrm{C}(\mathrm{b})$. Increasing the temperature to $8^{\circ} \mathrm{C}(\mathrm{c})$ causes the majority of the broad signal to diminish, leaving the narrow component. Figures were reproduced from Dosseh et al. ${ }^{40}$ Used under fair use, 2013. 


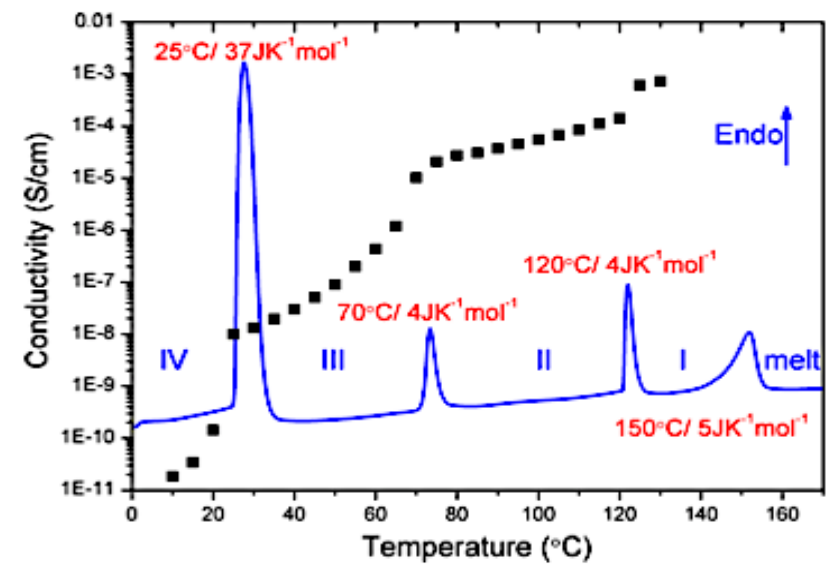

(a)

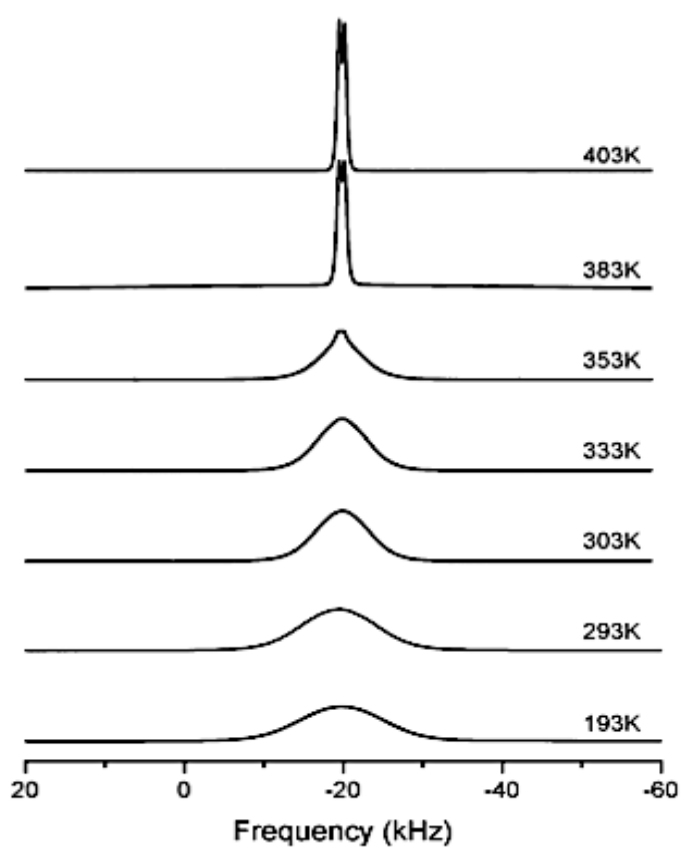

(b)

Figure 1.8: DSC thermogram (a), conductivity inset (a), and ${ }^{19} \mathrm{~F}$ variable temperature SSNMR results (b) for the OIPC $\left[\mathrm{P}_{1,2,2,4}\right]\left[\mathrm{PF}_{6}\right]$. DSC results show large $\Delta \mathrm{H}$ values at each phase transition, with a $\Delta S_{f}$ value of $5 \mathrm{~J} \mathrm{~mol}^{-1} \mathrm{~K}^{-1}$. The variable temperature ionic conductivity inset (a) displays a step-wise increase at each phase transition before reaching a final value on the order of $10^{-3} \mathrm{~S} \mathrm{~cm}^{-1}$. The ${ }^{19} \mathrm{~F}$ SSNMR results (b) display a broad signal in phases IV and III, suggesting that the $\mathrm{PF}_{6}{ }^{-}$is not mobile enough to become isotropically averaged. However, in phase II, a narrow signal now resides on top of the broad signal, suggesting that there is diffusive $\mathrm{PF}_{6}$. Figures reproduced from Jin et $a l .{ }^{31}$ Used under fair use, 2013.

Recently, Jin et al. have reported results from DSC (Figure 1.8a), impedance spectroscopy (Figure 1.8a), and multinuclear SSNMR (Figure 1.8b) for the OIPC diethyl(methyl)(isobutyl)phosphonium hexafluorophosphate $\left(\left[\mathrm{P}_{1,2,2,4}\right]\left[\mathrm{PF}_{6}\right]\right)\left(\right.$ Scheme 6). ${ }^{31}$ 

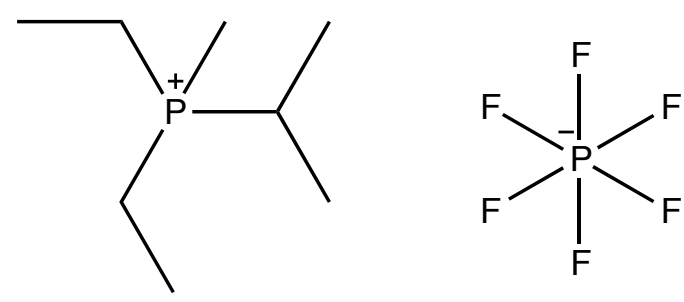

Scheme 6: Chemical structure of $\left(\left[\mathrm{P}_{1,2,2,4}\right]\left[\mathrm{PF}_{6}\right]\right){ }^{31}$

Specifically, the variable temperature ${ }^{19} \mathrm{~F}$ NMR spectra displays spectral features similar to those of Dosseh et al. ${ }^{40}$ and our variable temperature ${ }^{19} \mathrm{~F}$ NMR spectra (discussed in Chapter 3); a narrow component $\left(J_{\mathrm{PF}}=720 \mathrm{~Hz}\right)$ resides on top of a broad component. The narrow signal is consistent with a $J_{\mathrm{PF}}$ of $\sim 720 \mathrm{~Hz}$, which was determined from a standard solution of $\mathrm{KPF}_{6}$, discussed in Chapter 3. Jin et al. suggested the narrow component is diffusive $\mathrm{PF}_{6}^{-}$in phase II (Figure $1.8 \mathrm{~b}, 353$ and $383 \mathrm{~K}$ ). Interestingly, the variable temperature ${ }^{19} \mathrm{~F}$ NMR data displays an isotropic signal (at $403 \mathrm{~K}$ ) well below $T_{m}(423 \mathrm{~K})$. Jin et al. were able to determine the amount of mobile $\mathrm{PF}_{6}{ }^{-}$as a function of temperature; a step-wise decrease in mobile $\mathrm{PF}_{6}{ }^{-}$going from phase I (100 mol \% mobile $\left.\mathrm{PF}_{6}^{-}\right)$to III (10 mol \% mobile $\left.\mathrm{PF}_{6}^{-}\right)$was observed. The authors suggested ion hopping in phase III with $1 \mathrm{~mol} \%$ cation and $10 \mathrm{~mol} \%$ anion mobile. Isotropic mobility of the anion in phase II and I governs the conduction, as evidenced by the narrow NMR line widths. The variable temperature ${ }^{1} \mathrm{H}$ and ${ }^{19} \mathrm{~F}$ NMR results Dosseh et al. ${ }^{40}$ and Jin et al. ${ }^{31}$ reported, respectively, are interesting to note, as both are similar in the sense that a narrow NMR signal on top of a broad NMR signal is observed. Such spectral features potentially provide insight to the role of the grain boundary with respect to the conduction of ions in polycrystalline materials. Now that we have discussed in detail the various types of defects commonly present in a 
crystalline lattice and a polycrystalline material, we will turn our focus to what occurs in such materials when doped with small ions, such as $\mathrm{Li}^{+}$and $\mathrm{H}^{+} .41$

Doping small ions became of interest due to large increases, by several orders of magnitude, in conductivity upon doping. ${ }^{13-15,26,42}$ Conductivity is sometimes increased as a function of increased defects, ${ }^{15}$ which may potentially form from the doping of small ions into the crystalline lattice. Much research has gone into the doping of small ions to give highly conductive OIPCs, but little is known about the transport mechanism of the dopant, thus setting precedence to better understand the transport properties of these materials, as it is believed the dopant is the charge-carrying species for conduction. MacFarlane et al. and Forsyth et al. have led in doping $\mathrm{Li}^{+}$salts into OIPCs such as $\left[\mathrm{P}_{12} \mathrm{TFSI}\right]$ and $\left[\mathrm{P}_{11} \mathrm{TFSI}\right]$, respectively. ${ }^{13,15}$ They tested the hypothesis that doping of $\mathrm{Li}^{+}$into these OIPCs would increase conductivity. ${ }^{13}$ Increases in conductivity, from pure OIPCs to doped, with conductivity values on the order of $10^{-4} \mathrm{~S} \mathrm{~cm}^{-1}$ at room temperature were reported. ${ }^{13,15}$ MacFarlane et al. employed variable temperature ${ }^{7} \mathrm{Li}$ NMR (Figure 1.9b) on Li-doped $\left[\mathrm{P}_{12}\right.$ TFSA] and observed a narrowing of the $\mathrm{Li}^{+}$signal at $\sim 300 \mathrm{~K}$ (Figure 1.9a). It was suggested that a secondary melt phase with $9.3 \mathrm{~mol} \% \mathrm{Li}^{+}$was formed. ${ }^{13}$ Interestingly, a narrow component on top of a broad component can be seen at $267 \mathrm{~K}$ and $291 \mathrm{~K}$, suggestive of $\mathrm{Li}^{+}$within two distinct environments. The narrow component is $\mathrm{Li}^{+}$in the grain boundary and the broad component is $\mathrm{Li}^{+}$in the crystallite. Recall, previous work by Dosseh et $a l .{ }^{40}$ and Jin et al. ${ }^{31}$ showed similar spectral features (Figures 1.7 and $1.8 \mathrm{~b}$, respectively), and Dosseh et al. suggested the narrow component was material in the grain boundary and the broad component was crystalline material. ${ }^{40} \mathrm{Jin}$ et al. attributed the conductivity of the material to ion hopping of the anion in phase III (10 mol \% mobile anion) to phase I (100 mol \% mobile anion), as evidenced by the narrow NMR line widths below $T_{\mathrm{m}}{ }^{31}$ Although the studies by Dosseh et al 
and Jin et al. were not focused on the doping of small ions into the native lattice, similar NMR spectral features (i.e., distinct narrow and broad component) may potentially give insight into various components of the bulk material and how nanometer to micron-size defects (i.e. grain boundaries) play a role in ion transport.

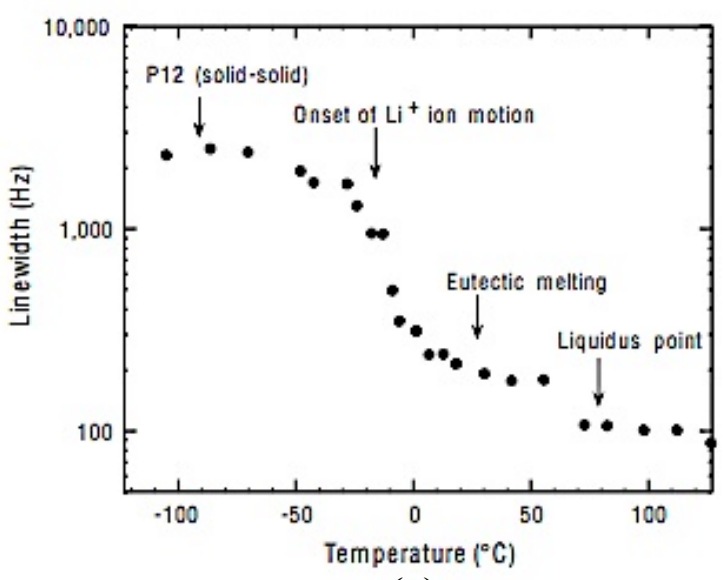

(a)

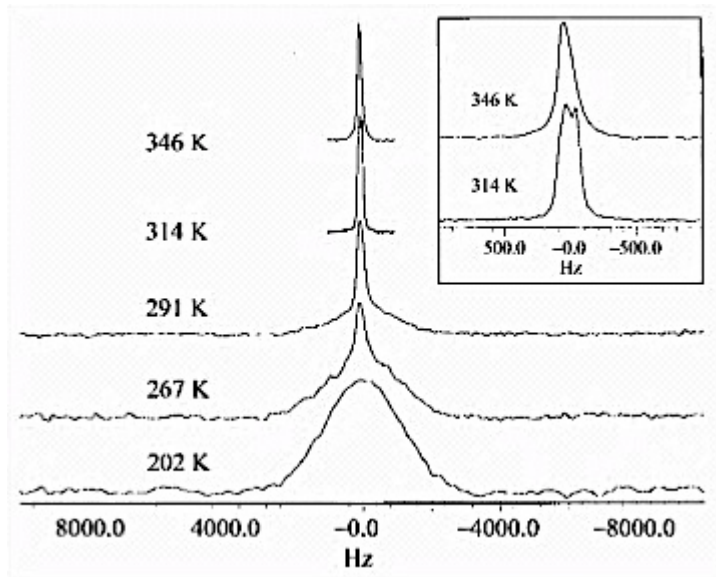

(b)

Figure 1.9: Stacked ${ }^{7} \mathrm{Li}$ NMR spectra as a function of temperature for the OIPC $\left[\mathrm{P}_{12} \mathrm{TFSI}\right]$ doped with $9.3 \mathrm{~mol} \% \mathrm{Li}^{+}$(b), $\mathrm{Li}^{+}$mobility is observed by the narrowing of the NMR signal (a) (i.e., decrease in line width), which is indicative of highly mobile $\mathrm{Li}^{+}$. A eutectic phase is observed below $T_{m}$, suggestive of a secondary melt phase, suggested to be mobile $\mathrm{Li}^{+}$, which is in good agreement with the high ionic conductivity observed for $\left[\mathrm{P}_{12} \mathrm{TFSI}\right]$. Figures reproduced from MacFarlane et al. ${ }^{13}$ Used under fair use, 2013.

The concentration of dopant ion should be considered. It has been shown that a dopant concentration threshold exists such that a new dopant rich liquid phase forms beyond that threshold while shifting the intrinsic phase transitions of the original material (i.e., the phase transitions may increase or decrease in temperature). ${ }^{26,43}$ Adebahr et al. investigated $N, N-$ 
dimethylpyrrolidinium iodide, $\left[\mathrm{C}_{1} \mathrm{mpyr}\right][\mathrm{I}]$ (Scheme 7) (It should be noted that Adebahr et al. used a different abbreviation in naming the pyrrolidinium family cations in this manuscript and changed to the $\mathrm{P}_{\mathrm{xy}}{ }^{+}$where $\mathrm{x} / \mathrm{y}=1,2,3$,etc. form in the manuscript by Huang et al. ${ }^{33}$ ), doped with 2, 5, and $10 \mathrm{wt} \% \mathrm{LiI}^{26}{ }^{26}$ 's of $476 \mathrm{~K}$ and $473 \mathrm{~K}$ were observed for the pure and doped (10 wt $\%$ LiI) OIPC, respectively. ${ }^{26}$

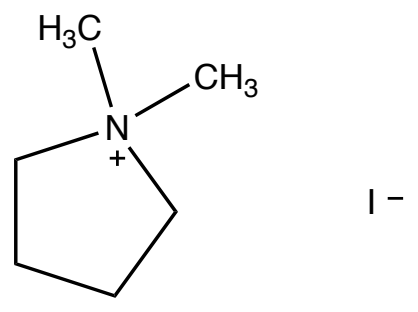

Scheme 7: Chemical structure of $\left[\mathrm{C}_{1} \mathrm{mpyr}\right][\mathrm{I}] .{ }^{26}$

Interestingly, $T_{m}$ for the pure OIPC is shifted to a lower temperature upon the doping of $\mathrm{Li}^{+}$, with an increase in conductivity by three orders of magnitude $\left(10^{-6}\right.$ to $\left.10^{-3} \mathrm{~S} \mathrm{~cm}^{-1}\right)$; moreover, the doping of LiI into $\left[\mathrm{C}_{1} \mathrm{mpyr}\right][\mathrm{I}]$ exhibits two-component conductivity. The sudden increase in mobility (larger conductivity component) accompanied by the lowering in $T_{m}$ for the doped OIPC was suggested to be due to the conduction of $\mathrm{Li}^{+}$through the crystalline lattice. More specifically, the lowering in $T_{m}$ for the doped OIPC was suggested to be due to an increase of $\mathrm{C}_{1} \mathrm{mpyr}^{+}$in the grain boundary (smaller conductivity component). ${ }^{26}$ Figures $1.10 \mathrm{a}$ and $1.10 \mathrm{~b}$ show stacked ${ }^{1} \mathrm{H}$ NMR spectra as a function of temperature for the pure OIPC and doped OIPC, respectively; emphasizing that there is a change in some fraction of the cation environment upon the addition of LiI. The solid-solid phase transition at $370 \mathrm{~K}$ in the pure OIPC (Figure 1.10a) shows a broad signal $(\sim 10 \mathrm{kHz})$, suggestive of cations within a rigid environment, such as a crystallite. However, upon doping (Figure 1.10b), a narrow signal on top of a broad signal is 
observed at the same phase transition. Adebahr et al. suggested that the narrow component was due to $\sim 20 \%$ of $\mathrm{C}_{1}$ mpyr $^{+}$residing in the grain boundary at the phase III to II transition, and was due to the smaller conductivity component. ${ }^{26}$ The authors also mentioned the use of ${ }^{125}$ I studies, but translational information could not be obtained; thus iodide could not be ruled out as a contributor to conductivity. ${ }^{26}$ 


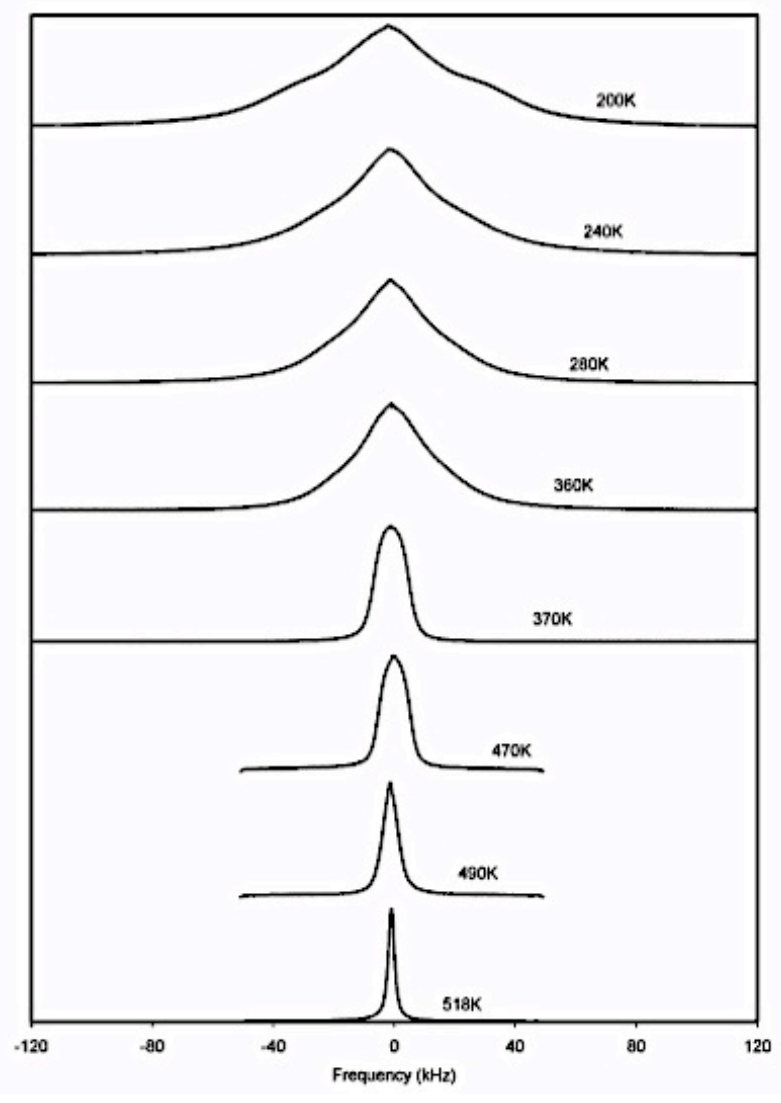

(a)

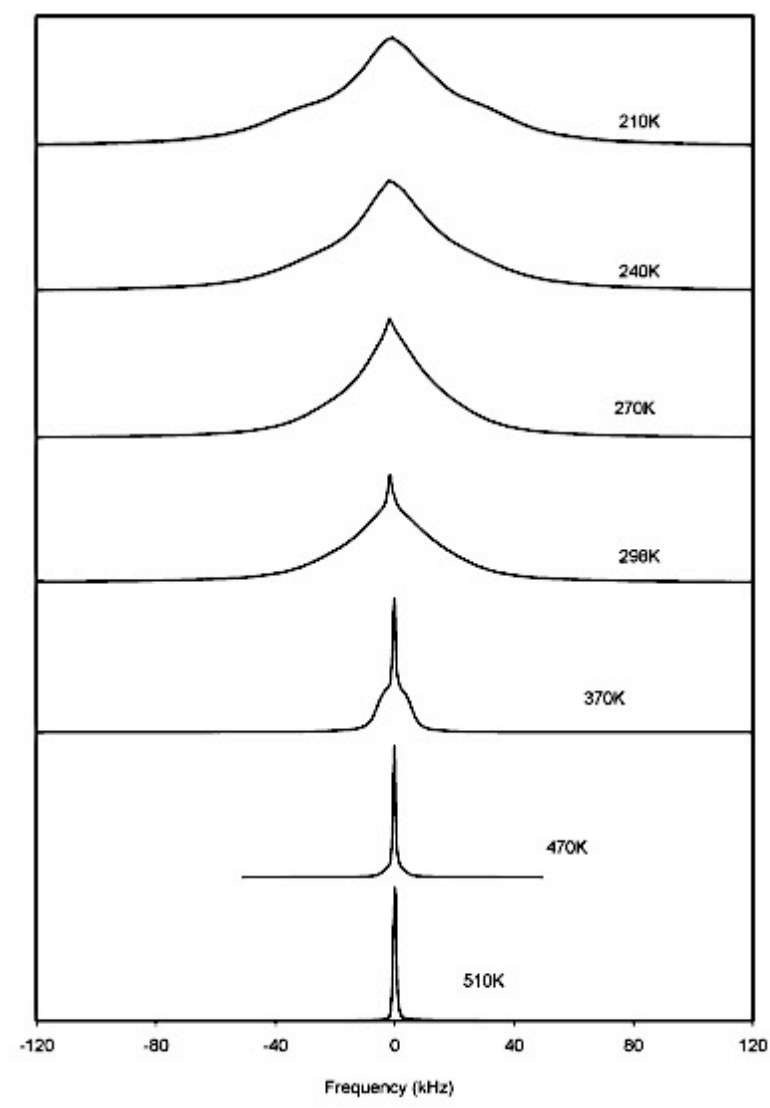

(b)

Figure 1.10: Stacked ${ }^{1} \mathrm{H}$ NMR spectra as a function of temperature for the pure OIPC (a), $\left[\mathrm{C}_{1} \mathrm{mpyr}\right][\mathrm{I}]$, and doped OIPC with $10 \mathrm{~mol} \% \mathrm{LiI}(\mathrm{b})$. Doping of LiI causes $\sim 20 \% \mathrm{C}_{1} \mathrm{mpyr}^{+}$to reside in the grain boundary, as seen in (b) at $370 \mathrm{~K}$ (near phase III to II transition) where a narrow ${ }^{1} \mathrm{H}$ signal resides on top of a broad signal. Upon doping, $T_{m}$ was decreased from $476 \mathrm{~K}$ to $473 \mathrm{~K}$, accompanied by an increase in ionic conductivity, most likely due to $\mathrm{C}_{1}$ mpyr ${ }^{+}$diffusing through the grain boundary. Figures reproduced from Adebahr et al. ${ }^{26}$ Used under fair use, 2013.

Studies by Rana et al. have shown that the doping of $\mathrm{H}_{3} \mathrm{PO}_{4}$ into OIPC choline dihydrogen phosphate, [Choline][DHP] (Scheme 8), resulted in conductivities on the order of $10^{-3} \mathrm{~S} \mathrm{~cm}^{-1}$. ${ }^{4}$ 

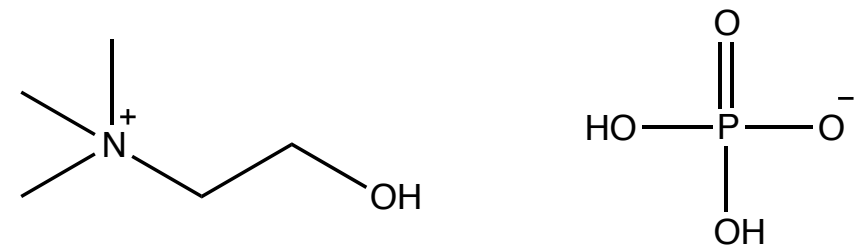

Scheme 8: Chemical structure of [Choline][DHP]. ${ }^{43}$

The amount of $\mathrm{H}_{3} \mathrm{PO}_{4}$ needed to produce such conductivities reached $\sim 18 \mathrm{~mol} \% \mathrm{H}_{3} \mathrm{PO}_{4}$, which created a "soft" multi-phase material. ${ }^{43}$ More recently, Rana et al. observed high proton conductivities from doping with strong acids, such as trifluoromethanesulfonic acid (TfOH) and bis-trifluoromethanesulfonyl imide $\left[\mathrm{HN}(\mathrm{Tf})_{2}\right]$ into [Choline $][\mathrm{DHP}] .{ }^{41,44}$ Similar to their previous results, an amorphous phase formed upon doping of $8-10 \mathrm{~mol} \% \mathrm{TfOH}$. The amorphous phase Rana et al. reported was observed through powder XRD studies at $22{ }^{\circ} \mathrm{C}$ where sharp Bragg reflections reside on top of a small broad component in the $\theta=18-30^{\circ}$ range. ${ }^{41}$ Although it was not explicitly stated, the amorphous phase observed by Rana et al. could potentially be the dopant acid residing in the grain boundary.

An overview of crystalline defects has been provided and how each influences ion transport not only in ionic solids, but also in polycrystalline materials. Schottky defects, grain boundaries, and doping with small ions construct the local environment that governs ion transport in OIPCs. However, it is impossible to predict which form(s) of disorder will be present and dominate the conduction mechanism within each solid phase below $T_{\mathrm{m}}$. With a large dependency on the cation-anion pair, precedence is set for detailed studies on new and existing materials. Insight into such properties is needed in order to fully optimize and take advantage of ion transport in such materials. 


\subsection{Molecular Diffusion and $E_{a}$}

One way to describe diffusion is through Fick's laws, whereby a negative concentration gradient drives particles from a high concentration to a low concentration region until equilibrium. ${ }^{32}$ Holding true for some systems, Fick's laws are insufficient to describe the diffusion of a homogenous system in the absence of a concentration gradient, where one of the main driving forces for diffusion is $k T$, the average thermal energy. During the diffusion process, a given particle receives "thermal kicks" from neighboring particles, thus resulting in a "random walk." The random walk of a particle is a well-known phenomenon that was discovered by Robert Brown and is fundamental to all chemistry. Commonly referred to as "Brownian motion," it describes the random translational mobility of any molecular species throughout a given environment. ${ }^{32}$

A simple illustration of Brownian motion is shown in Figure 1.11. Equation 1.1 describes the root mean square displacement of a particle diffusing in a $3-\mathrm{D}$ system $(\mathrm{x}, \mathrm{y}$, and $\mathrm{z}$ coordinates), where $\langle r\rangle$ is the displacement, $D$ is the diffusion coefficient, and $t$ is the diffusion time. 


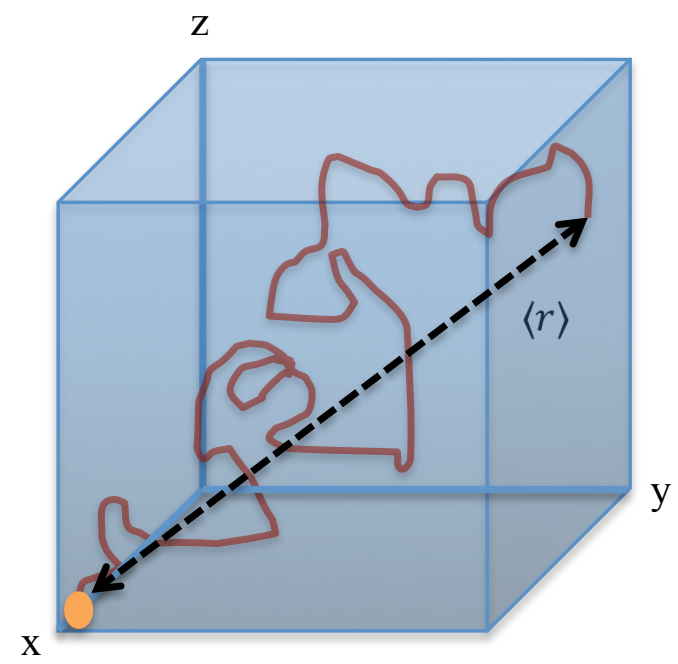

Figure 1.11: Illustration of the Brownian motion a particle experiences due to the thermal "kicks" the particle receives from neighboring particles. The experimentally measured diffusion coefficient $D$ is dependent on the average displacement of the particle as a function of time $t$.

$$
\langle r\rangle=\sqrt{6 D t}
$$

The diffusion of particles will be dependent on not only $k T$, but also on the local environment (i.e., the number of degrees of freedom) surrounding the diffusive species. We can think of the random walk, for a single particle, being governed by thermal "kicks" that particle receives from neighboring particles as it diffuses through the system (i.e., generating a random walk), which is dependent on the potential and kinetic energy of each individual particle in the system. Both $k T$ and diffusion are tightly correlated, and one cannot consider diffusion without considering $k T$.

In conjunction with Brown's observations, Einstein ${ }^{45,46}$ proposed that the self-diffusion coefficient of a spherical particle in an infinitely dilute environment could be described by (Equation 1.2):32,46 


$$
D=\frac{k T}{f}
$$

Where $k T$ is the average thermal energy ( $k$ is Boltzmann's constant and $T$ is temperature) and $f$ is the referred to as the "frictional coefficient". Stokes later proposed that the frictional coefficient for a spherical particle with radius $r_{H}$ (commonly known as the hydrodynamic radius) in an environment with viscosity $\eta$ could be described by (Equation 1.3$):^{32,46}$

$$
f=6 \pi \eta r_{H}
$$

which led to the well-known Stokes-Einstein equation (Equation 1.4): ${ }^{32,46}$

$$
D=\frac{k T}{6 \pi \eta r_{H}}
$$

Thus a fundamental approach to understand the relationship between the self-diffusion coefficient of a diffusing species and the intrinsic properties of the environment encompassing the diffusing species was formulated. We see that the diffusion coefficient is inversely proportional to the product of the viscosity $\eta$ of the material and hydrodynamic radius $r_{H}$ of the diffusing species.

The diffusion coefficient is a powerful value that has gained much interest in all sciences, as it contains rich information about the molecular environment surrounding the diffusive species. ${ }^{3,6}$ Diffusion as a function of temperature allows one to extract the translational activation energy $E_{a}$ for a given system, as described by the Arrhenius equation (Equation 1.5), wherein $D$ 
is the diffusion coefficient, $D_{0}$ is the pre-exponential factor (the diffusion coefficient at infinite temperature), $E_{\mathrm{a}}$ is the translational activation energy, and $k T$ is the average thermal energy. Classically, $E_{\mathrm{a}}$ for a diffusing species is described as the minimum amount of energy needed for that species to jump from one lattice position to another ("ion hopping") through the crystalline lattice, providing there is a vacancy for the diffusing species to occupy (Figure 1.12). Figure 1.12 is an illustration of an ionic solid in which the cations (blue) are representative of a small ion, such as $\mathrm{Li}^{+}$, while the anions (red/yellow) are representative of a tetrahedral molecule, such as $\mathrm{SO}_{4}{ }^{2-}$.

$$
D=D_{0} e^{\frac{-E_{a}}{k T}}
$$
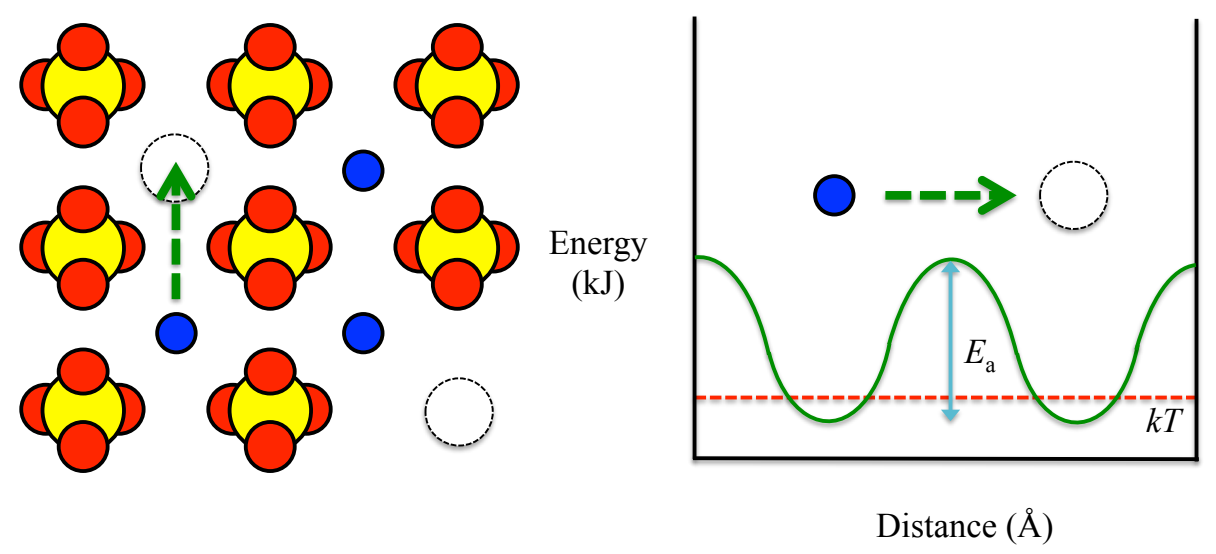

Figure 1.12: Expansion of Figure 1.3 allows us to illustrate an ionic compound, such as $\mathrm{Li}_{2} \mathrm{SO}_{4}$, where the cation (blue) "hops" from one lattice position to another past the anions (yellow/red) with some barrier $E_{\mathrm{a}}$.

Tightly correlated with $D$ and $E_{\mathrm{a}}, D_{0}$ gives insight into the local structure governing both properties. Classically termed the "pre-exponential factor" or "frequency factor", $D_{0}$ represents 
the configurational degrees of freedom for a given system under specific conditions. Intuitively we expect that a large $D$ will be accompanied by a small $E_{\mathrm{a}}$. However, such a relationship does not always hold true for all systems, as it will be shown that a small $D$ accompanied by a small $E_{\mathrm{a}}$ is also possible due to the properties of the surrounding system (i.e., steric hindrance and grain boundaries) and how they influence the diffusing species. Thus, the ion hopping mechanism may be insufficient in describing the ion transport for all ionic solids as the conducting species may not follow such a discrete mechanism.

Every et al. studied iodide and bromide salts of 1-methyl-3-ethylimidazolium (MeEtImI $T_{m}=351 \mathrm{~K}$ and MeEtImBr $T_{m}=352 \mathrm{~K}$, respectively) by means of DSC, ${ }^{1} \mathrm{H}$ fringe field gradient (FFG) and/or pulsed-field gradient (PFG) NMR, and modeled conductivity. ${ }^{29}$ Only MeEtImI displayed a solid-solid phase transition at $242 \mathrm{~K}$. They reported diffusion coefficients for the cation on the order of $10^{-10} \mathrm{~m}^{2} \mathrm{~s}^{-1}$ accompanied by $E_{\mathrm{a}}$ values of $20-30 \mathrm{~kJ} \mathrm{~mol}^{-1}$ and conductivity values on the order of $10^{-3} \mathrm{~S} \mathrm{~cm}^{-1}$ at room temperature $\left(25^{\circ} \mathrm{C}\right) .{ }^{127} \mathrm{I}$ and bromine (isotope was not reported) NMR results were not obtained due to the short relaxation times (i.e., $T_{1}$ and $T_{2}$ ), thus making it experimentally difficult to obtain self-diffusion coefficients, suggesting that the anions were in a rigid environment. From their variable temperature measured diffusion coefficients and conductivity values, it was suggested that two environments for the cation existed in the solidstate, one immobile and the other mobile. The immobile environment was suggested to be cations that maintained the crystalline lattice and the diffusive environment was suggested to be cations diffusing through the crystalline lattice. Similar spectral features as Jin et al. and Dosseh et al. (i.e., narrow and broad component) could potentially exist for this system, however, Every et al. noted that experimentally distinguishing these with a diffusion experiment (discussed in subsequent chapters) would be difficult, as the time-scale of the immobile cation (i.e., 
crystalline) would be too short for their NMR measurements, thus concluding why only the mobile species was spectroscopically observed. It would be interesting to see the 1-D ${ }^{1} \mathrm{H}$ NMR data; as such an experiment does not produce a $T_{2}$-limited spectrum (discussed in subsequent chapters). The transport mechanism was said to occur through the "sliding" motion of cations through the crystalline lattice. However, in their discussion it was noted that the van der Waals radii would make it quite difficult for such motions to occur in the crystal structure for both salts (i.e., steric hindrance). They observed a change in cation $E_{\mathrm{a}}$ near $T_{m}$, suggesting a change in the cation transport mechanism but did not observe a step-wise increase in diffusion coefficients and conductivity values. ${ }^{29}$ It should be noted that Every et al. determined conductivity values from diffusion coefficients using the Nernst-Einstein equation (Equation 1.6), ${ }^{29}$ wherein $\sigma$ is conductivity, $D_{+/}$is the diffusion coefficient for the cation and anion, $c_{+/-}$is the concentration of the conductive charge carriers, $q$ is the ionic charge, $k$ is Boltzmann's constant, and $T$ is temperature. It can be seen that conductivity is largely dependent on the diffusion coefficient and concentration of the charge carrying species.

$$
\sigma=\frac{\left(D_{+} c_{+}+D_{-} c_{-}\right) q^{2}}{k T}
$$

It has been suggested that grain boundaries inhibit the conduction of ions. ${ }^{10}$ The grain boundary may allow fast diffusion as a result of a decrease in steric hindrance and an increase in the configurational degrees of freedom; however, the concentration of charge carrying species may be so low that the measured conductivity is inherently low. 
Revisiting the recent work by Jin et al. on the OIPC $\left(\left[\mathrm{P}_{1,2,2,4}\right]\left[\mathrm{PF}_{6}\right]\right), E_{\text {a }}$ values of 74,43 , and $20 \mathrm{~kJ} \mathrm{~mol}^{-1}$ were reported for phases IV, III, and II, respectively. Jin et al. also reported selfdiffusion coefficients on the order of $10^{-11} \mathrm{~m}^{2} \mathrm{~s}^{-1}$ for the cation and anion as a function of temperature. Self-diffusion coefficients have proven to be very powerful in understanding transport properties of small ions and molecules through various materials. ${ }^{3,4,6,47}$ However, Jin et al. determined diffusion coefficients by means of measuring the full-width at half-maximum (in Hertz) of the ${ }^{1} \mathrm{H}$ and ${ }^{19} \mathrm{~F}$ NMR narrow signal on top of the broad signal (i.e., the narrow signal in Figure $1.8 \mathrm{~b}$ ) and using these values to characterize motional properties (i.e., self-diffusion coefficient). The use of an externally applied magnetic field gradient provides a direct measurement of the self-diffusion coefficient ${ }^{48}$ for cations and anions separately, as will be shown in subsequent chapters. Although Jin et al. suggested ion hopping through the crystalline lattice to be the transport mechanism for the diffusive $\mathrm{PF}_{6}^{-}$, they also suggested that the material behaved like an IL in phase II, as evidenced by the low $E_{\mathrm{a}}$ value. ${ }^{31}$ It is hard to envisage such a low $E_{\mathrm{a}}$ within the crystalline lattice if one considers steric hindrance, unless there were large concentrations of cations and anions diffusing in a second environment (i.e., grain boundary) close to $T_{\mathrm{m}}$. Jin et al. did mention a second smaller component for conduction that was suggested to be the native cation diffusing in the grain boundary. An $E_{\mathrm{a}}$ value of $20 \mathrm{~kJ} \mathrm{~mol}^{-1}$ is very close to that of pure water $\left(18 \mathrm{~kJ} \mathrm{~mol}^{-1}\right),{ }^{6}$ suggestive of liquid-like energetics within a solid phase.

Diffusion studies on OIPCs have been conducted by means of NMR line width analysis, PFG NMR, and conductivity studies. For the systems reported in this thesis, the majority of $E_{\mathrm{a}}$ values have been calculated from conductivity values, which were experimentally determined or modeled. Impedance spectroscopy is a bulk measurement and lacks the ability to report on individual ions in an ionic system (unlike NMR which has the advantage of chemical and nuclear 
specificity), thus only speculations may be made as to which ion contributes to the conductivity. It has also been suggested that grain boundary diffusion occurs in these materials. The majority of literature, outside of what has been presented, suggests ion hopping in the crystalline lattice to be the major transport mechanism for conduction. While these results provide powerful descriptions for ion transport within OIPCs, no clear description of grain boundary diffusion exists. It is possible that ion hopping occurs through Schottky-type defects for a cation-anion pair composed of small ions, such as $\mathrm{H}^{+}$and $\mathrm{Li}^{+}$, which are small enough to diffuse through the crystalline lattice. However, for a bulky cation-anion pair with steric hindrance, conductivity values on the order of $10^{-3} \mathrm{~S} \mathrm{~cm}^{-1}$ in the crystalline lattice are hard to imagine without an alternate description.

\subsection{Joint Rotational and Translational Motion}

Briefly mentioned thus far, short-range disorder within OIPCs is also attributed to the rotator phase. The term "rotator phase" has been coined for the rotational motion experienced by molecules in the crystalline lattice at a solid-solid phase transition below $T_{m}$. Low $\Delta S_{f}$ 's are observed for OIPCs due to the large enthalpy changes observed at these phase transitions and is believed to be due, in part, to the onset of isotropic rotation of the cation and/or about one or more axes. Once rotational motion begins in the crystalline lattice, the entropy of the solid state increases closer to that of the liquid state, causing $\Delta S_{f}$ to be small when compared to a fully ordered crystalline lattice. The rotator phase is a very complex component of an OIPC, which may also promote ion transport. Being temperature dependent, it is difficult to identify rotator phases without understanding the phase behavior of the material. A physical description of a rotator phase is needed to deepen the fundamental knowledge of ion transport within OIPCs, as ion transport within an OIPC is sometimes enhanced within a rotator phase. 
So far we have discussed ion transport in the crystalline lattice in the absence of adjacent rotating counterions. Like diffusion, rotational motion is also temperature dependent and has some barrier $E_{\mathrm{a}}$ associated with it. Figure 1.13 is an illustration of an ionic solid in which the cation (blue) is representative of a small ion, such as $\mathrm{Li}^{+}$, while the anions (red/yellow) are representative of a tetrahedral molecule, such as $\mathrm{SO}_{4}{ }^{2-12}$

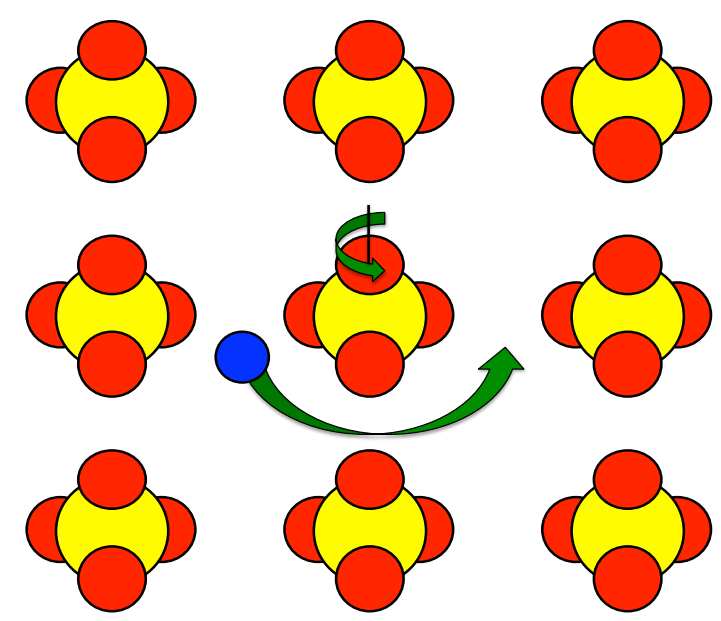

Figure 1.13: Illustration of the paddle-wheel mechanism where the revolving molecule is $\mathrm{SO}_{4}{ }^{2-}$ and the small translating ion is $\mathrm{Li}^{+}$. The rotation of $\mathrm{SO}_{4}{ }^{2-}$ can lower the barrier to translation for $\mathrm{Li}^{+}$, which can influence the transport of $\mathrm{Li}^{+}$into a vacancy. The remaining cations within the lattice were intentionally not shown to emphasize the joint rotational and translational motion. Figure adapted from MacFarlane et $_{\text {al. }}{ }^{12}$

As previously mentioned, ion hopping within the crystallite may potentially be influenced by the rotation of the counterion. The rotation of $\mathrm{SO}_{4}{ }^{2-}$ acts as a swinging door, making translational mobility for $\mathrm{Li}^{+}$more energetically favorable to travel through the crystalline lattice, ${ }^{49}$ assuming there are vacancies present for $\mathrm{Li}^{+}$to occupy. The joint rotational and translational motion of ions in a crystalline lattice is referred to as the "paddle-wheel" 
mechanism. ${ }^{10,11}$ Kvist and Bengtzelius et al. first proposed the paddle-wheel mechanism in 1969 when it was shown that $\mathrm{SO}_{4}{ }^{2-}$ in $\alpha-\mathrm{Li}_{2} \mathrm{SO}_{4}$ rotates freely about its fixed crystalline lattice point upon entering a rotator phase at $575{ }^{\circ} \mathrm{C}$, thus allowing easier $\mathrm{Li}^{+}$diffusion through the lattice. ${ }^{9}$ The paddle-wheel behavior may become more probable as temperature is increased, due to thermal expansion of the crystallite, decreasing the steric hindrance between the cation and anion. If an OIPC enters a rotator phase, it is possible that an increase in the number of mobile ions occurs due to the decrease in translational $E_{\mathrm{a}}$ (Figure 1.14).

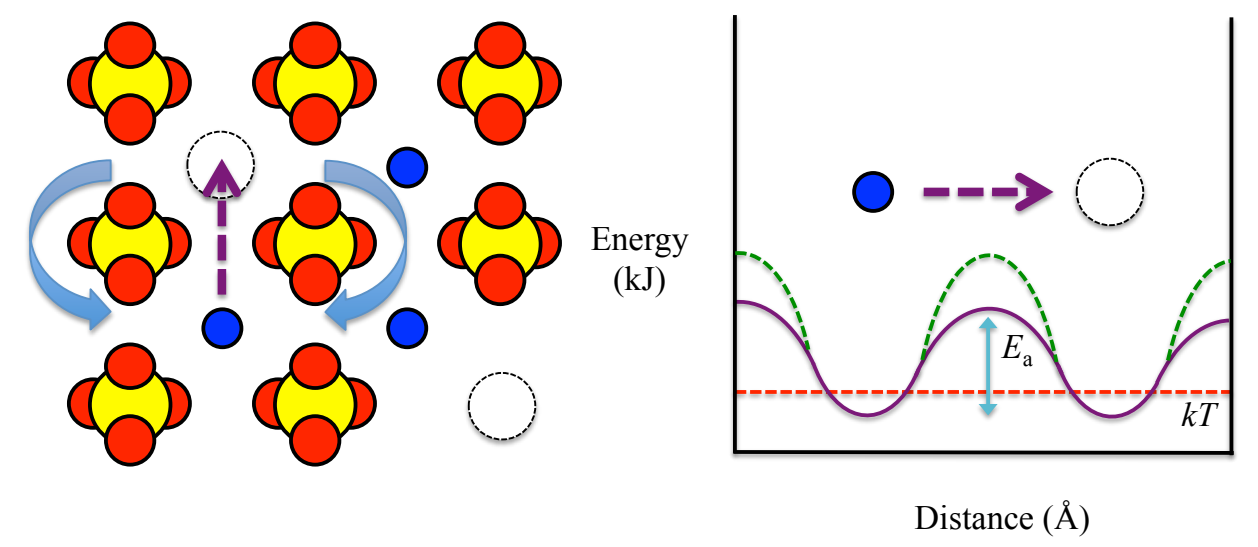

Figure 1.14: Expansion of Figure 1.12 illustrates that upon rotation of the anion (yellow/red), the barrier to translation for the cation (blue) decreases. We can rationalize the lowering of the barrier to translation for the cation due to the crystallite volume expansion at a first-order phase transition that is commonly observed for OIPCs. The volume expansion of the crystallite may promote rotation of the cation and/or anion and thus decreases the translational $E_{\text {a }}$ of the counterion.

Aronsson et al. followed up the studies of Kvist and Bengtzelius and confirmed that $\alpha-\mathrm{Li}_{2} \mathrm{SO}_{4}$ went from a fully ordered crystalline phase to a rotator phase at $575{ }^{\circ} \mathrm{C}$, where the onset of 
rotational motion of $\mathrm{SO}_{4}{ }^{2-}$ was observed with Brillouin scattering. They showed that $\mathrm{SO}_{4}{ }^{2-}$ displays paddle-wheel behavior, allowing the transport of $\mathrm{Li}^{+}$through the crystalline lattice. A conductivity value on the order of $10^{-6} \mathrm{~S} \mathrm{~cm}^{-1}$ was attributed to the transport of $\mathrm{Li}^{+}$ions. A similar study of $\alpha-\mathrm{Na}_{3} \mathrm{PO}_{4}$ by Wilmer et al. showed, ${ }^{50}$ by means of neutron scattering, that anion rotation was restricted about one of the $C_{3}$-axes with a barrier to rotation of $17.8 \mathrm{~kJ} \mathrm{~mol}^{-1}$. Conductivity on the order of $10^{-3} \mathrm{~S} \mathrm{~cm}^{-1}$ was also observed upon entering a rotator phase at 325 ${ }^{\circ} \mathrm{C} .{ }^{50}$ Recently, Cahill et al. have used variable temperature ${ }^{1} \mathrm{H}$ NMR to study the $\mathrm{H}^{+}$transport mechanism in the OIPC $[$ Choline $][\mathrm{DHP}],{ }^{51}$ in conjunction with the studies by Rana et al. ${ }^{43}$ discussed earlier. Cahill reported $\mathrm{H}^{+}$exchange from [DHP]--to-[DHP] $]^{-}$through the rotation of $[\mathrm{DHP}]^{-}$about it's fixed crystalline lattice point, ${ }^{51}$ which is consistent with the paddle-wheel mechanism.

The molecular geometry of the ion could play a role in the ability for an ion to rotate in the crystallite and ultimately the ability to translate. The tetrahedral geometry of $\mathrm{SO}_{4}{ }^{2-}$ could potentially rationalize why Kvist and Bengtzelius suggested the paddle-wheel mechanism. ${ }^{9}$ Being quasi-spherical in shape upon rotation decreases the steric hindrance with the cation and potentially decreases the barrier to rotation that may prevent the anion from freely rotating, depending on the counterion. Like $\mathrm{SO}_{4}{ }^{2-}, \mathrm{PO}_{4}{ }^{3-}$ also has a tetrahedral geometry and upon rotation is sterically less hindered to rotate about its fixed crystalline lattice point. $\mathrm{PF}_{6}{ }^{-}$has an octahedral geometry and is also quasi-spherical in shape upon rotation. Considering the geometry of $\mathrm{PF}_{6}^{-}$and applying it to a derivative of 1,2-bis[ $N$ - $\left(N^{\prime}\right.$-hexylimidazolium$\left.\left.d_{2}(4,5)\right)\right] \mathrm{C}_{2} \mathrm{H}_{4} 2 \mathrm{PF}_{6}^{-}, 1,2$-bis $\left[N-\left(N^{\prime}\right.\right.$-butylimidazolium- $\left.\left.d_{2}(4,5)\right)\right] \mathrm{C}_{2} \mathrm{H}_{4} 2 \mathrm{PF}_{6}^{-}$, the crystal structure of the latter gives some insight to the molecular environment of the $\mathrm{PF}_{6}^{-}$, as seen in Figure 1.15. Looking at Figure 1.15, it appears $\mathrm{PF}_{6}^{-}$resides in a "cup" formed by the alkyl chains and 
imidazolium rings where the rotation of $\mathrm{PF}_{6}^{-}$can be envisaged. Due to steric hindrance and the many hydrogen - fluorine contacts present, ${ }^{17}$ it is hard to imagine that rotational motion of $\mathrm{PF}_{6}{ }^{-}$ will promote translational motion of the dication.

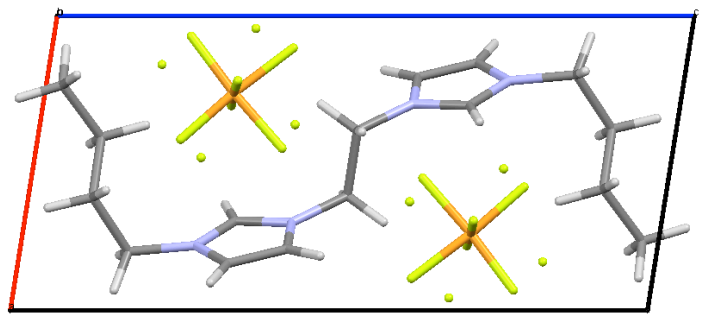

a

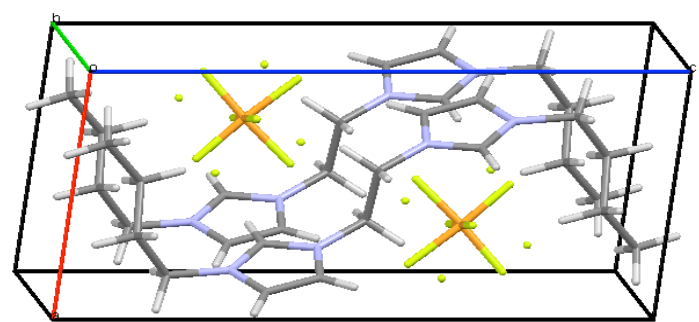

b

Figure 1.15: Illustration of the crystal structure for 1,2-bis[N-(N'-butylimidazolium$\left.\left.d_{2}(4,5)\right)\right] \mathrm{C}_{2} \mathrm{H}_{4} 2 \mathrm{PF}_{6}^{-}$viewed along the $b$-axis (a) and $b^{*}$-axis (b), showing the ease of rotational mobility for $\mathrm{PF}_{6}^{-}$. Figures reproduced from Lee et al. ${ }^{17}$ Used under fair use, 2013.

The rotator phase is of interest due to an observed increase in conductivity with respect to temperature, implying that rotational and translational motions are intimately related. Understanding the rotator phase of OIPCs is imperative in ultimately understanding the ion transport in such materials. Much research has gone into the elucidation of the rotator phase and its significance to conduction. Like ion transport, it is hard to predict which cation-anion pair will exhibit a rotator phase.

\subsection{Thermal History of OIPCs}

Thus far we have discussed in detail crystalline defects, the rotator phase, and how they relate to rotational and translational mobility within OIPCs. Barely mentioned in literature, and recently discovered, the thermal history also plays a large role in the bulk properties of these 
materials. Annealing can control the size and number of grain boundaries that form for polycrystalline materials. Rana et al. observed a decrease in conductivity $\left(10^{-5} \mathrm{~S} \mathrm{~cm}^{-1}\right)$ for pure [Choline][DHP] upon annealing the material at $100{ }^{\circ} \mathrm{C}$ for five days when compared to the doped material. ${ }^{41}$ Variable temperature ${ }^{1} \mathrm{H}$ NMR results (Figure 1.16) show a change in the material from the "as-prepared" polycrystalline material to the annealed material. The ${ }^{1} \mathrm{H} N M R$ results at $30{ }^{\circ} \mathrm{C}$ are different before and after annealing. The annealed spectrum shows a very broad signal appearing to be one component versus the "as-prepared" spectrum displays a narrow component residing on top of a broad component. At $100{ }^{\circ} \mathrm{C}$, the "as-prepared" and annealed spectra look similar; however, the "as-prepared" spectrum does not display as much broad signal as the annealed spectrum. Reported was a more uniform crystalline material after annealing versus what was synthesized. These results suggest that a carefully annealed polycrystalline material will be less defective (i.e., fewer and smaller grain boundaries) versus a freshly synthesized polycrystalline material. 

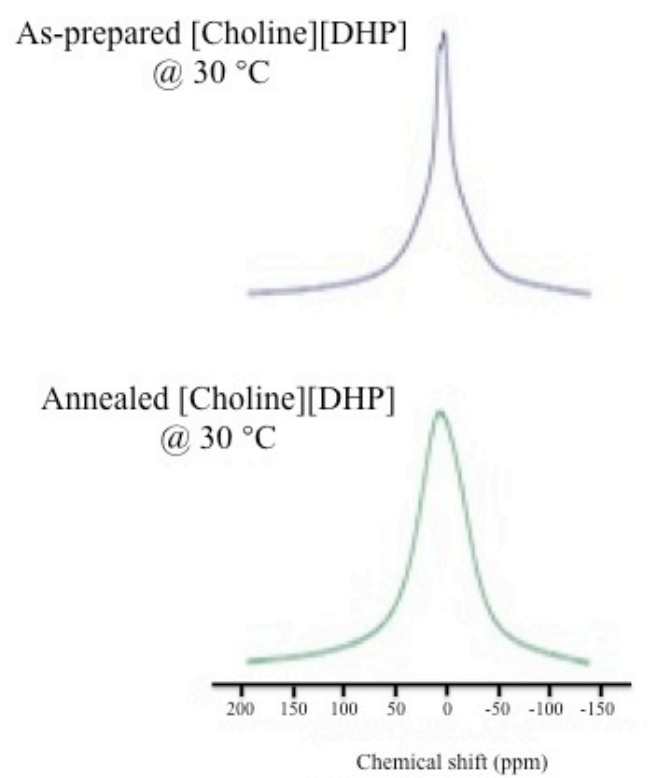
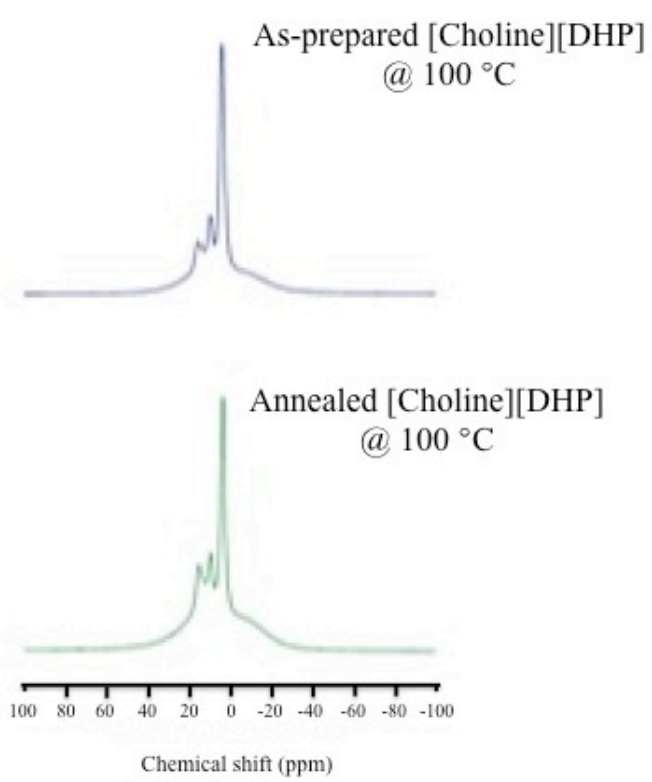

Figure 1.16: Variable temperature ${ }^{1} \mathrm{H}$ NMR spectra for the "as-prepared" and annealed OIPC [Choline][DHP]. The spectrum at $30{ }^{\circ} \mathrm{C}$ for the "as-prepared" material contains a narrow component on top of a broad component whereas the annealed material displays only a broad signal. ${ }^{1} \mathrm{H}$ NMR spectra for both "as-prepared" and annealed at $100{ }^{\circ} \mathrm{C}$ display similar spectral features; a broad and narrow component are observed. These results suggest that an annealed material is less defective and thus has fewer and smaller grain boundaries when compared to a pure material. ${ }^{41}$ Used under fair use, 2013.

Revisiting the work by Every et al. on the OIPCs MeEtImI and MeEtImBr, variable temperature PFG-NMR showed a change in diffusion coefficient for the cation when the material was melted and cooled in the magnetic field $\left(B_{0}\right)$ and then re-melted in $B_{0}$ again. Upon cooling lower than $T_{\mathrm{m}}$, a slow decrease in diffusion coefficient is observed. Interestingly, deviation from Arrhenius behavior (i.e., non-linear regression in the variable temperature diffusion data) is also observed to what the authors suggested to be a "supercooled liquid-state." A clear change in 
slope for the variable temperature cation diffusion data can be seen between the two data sets (not shown). ${ }^{29}$ Acquiring diffusion coefficients as the material was heated in $B_{0}$ gave diffusion coefficients much higher than when the material was cooled in $B_{0}$. Interestingly, a change in $E_{\mathrm{a}}$ (10-21 kJ mol ${ }^{-1}$ and $10-34 \mathrm{~kJ} \mathrm{~mol}^{-1}$ for MeEtImI and MeEtImBr, respectively) for the cation between the heating and cooling data sets was noted. ${ }^{29}$

As will be shown in Chapter 3, careful attention to the thermal history of an OIPC should be considered when studying transport properties in these materials. Moreover, varying temperature in the presence of a strong magnetic field can also alter the intrinsic transport properties.

\subsection{Concluding Remarks}

We will now turn our focus to the theory and methods of NMR, as the present study was conducted with an emphasis on novel NMR methods, such as variable temperature ${ }^{1} \mathrm{H} /{ }^{19} \mathrm{~F}$ PFG NMR and variable temperature ${ }^{1} \mathrm{H} /{ }^{19} \mathrm{~F} /{ }^{2} \mathrm{H}$ NMR. In conjunction with theory and current literature, Chapter 3 will present new and unique results for the OIPC 1,2-bis[ $\left[N-\left(N^{\prime}\right.\right.$ hexylimidazolium- $\left.\left.d_{2}(4,5)\right)\right] \mathrm{C}_{2} \mathrm{H}_{4} 2 \mathrm{PF}_{6}^{-}$.

\section{References:}

(1) Andújar, J. M.; Segura, F. Renewable and Sustainable Energy Rev. 2009, 13,

(2) Mauritz, K. A.; Moore, R. B. Chem. Rev. 2004, 104, 4535.

(3) Li, J.; Park, J. K.; Moore, R. B.; Madsen, L. A. Nat. Mater. 2011, 10, 507

(4) Li, J.; Wilmsmeyer, K. G.; Madsen, L. A. Macromolecules 2009, 42, 255.

(5) Li, J.; Wilmsmeyer, K. G.; Hou, J.; Madsen, L. A. Soft Mater. 2009.

(6) Lingwood, M. D.; Zhang, Z.; Kidd, B. E.; McCreary, K. B.; Hou, J.; Madsen, L. A. Chem. Commun. 2013.

(7) Parrondo, J.; Venkateswara Rao, C.; Ghatty, S. L.; Rambabu, B. Inter Electrochem 2011, 2011, 1. 
(8) Aronsson, R. J. Chem. Phys. 1982, 77, 677.

(9) Bengtzelius, A.; Kvist, A. Z. Naturforsch., A: Phys. Sci. 1969, 24, 468.

(10) Pringle, J. M. Phys. Chem. Chem. Phys. 2013, 15, 1339.

(11) Pringle, J. M.; Howlett, P. C.; MacFarlane, D. R.; Forsyth, M. J. Mater. Chem. 2010, 20, 2056.

(12) MacFarlane, D. R.; Forsyth, M. Adv. Mater. 2001, 13, 957.

(13) MacFarlane, D. R.; Huang, J. H.; Forsyth, M. Nature 1999, 402, 792.

(14) Zhou, Z. B.; Matsumoto, H. Electrochem. Commun. 2007, 9, 1017.

(15) Forsyth, M.; Huang, J.; MacFarlane, D. R. J. Mater. Chem. 2000, 10, 2259.

(16) Lee, M.; Choi, U. H.; Wi, S.; Slebodnick, C.; Colby, R. H.; Gibson, H. W. J. Mater. Chem. 2011, 21, 12280.

(17) Lee, M.; Niu, Z.; Slebodnick, C.; Gibson, H. W. J. Phys. Chem. B 2010, 114, 7312.

(18) Yu, G. R.; Zhao, D. C.; Wen, L.; Yang, S. D.; Chen, X. C. AIChE J. 2012, 58, 2885.

(19) Timmermans, J. J. Phys. Chem. Solids 1961, 18, 1.

(20) Pringle, J. M.; Shekibi, Y.; MacFarlane, D. R.; Forsyth, M. Electrochim. Acta 2010, 55, 8847.

(21) Howlett, P. C.; Sunarso, J.; Shekibi, Y.; Wasser, E.; Jin, L. Y.; MacFarlane, D. R.; Forsyth, M. Solid State Ionics 2011, 204, 73.

(22) Forsyth, S. A.; Fraser, K. J.; Howlett, P. C.; MacFarlane, D. R.; Forsyth, M. Green Chem. 2006, 8, 256.

(23) Adebahr, J.; Forsyth, M.; MacFarlane, D. R. Electrochim. Acta 2005, 50, 3853.

(24) Adebahr, J.; Grirnsley, M.; Rocher, N. M.; MacFarlane, D. R.; Forsyth, M. Solid State Ionics 2008, 178, 1798.

(25) Adebahr, J.; Johansson, P.; Jacobsson, P.; MacFarlane, D. R.; Forsyth, M. Electrochim. Acta 2003, 48, 2283.

(26) Adebahr, J.; Seeber, A. J.; MacFarlane, D. R.; Forsyth, M. J. Phys. Chem. B 2005, 109, 20087.

(27) Efthimiadis, J.; Forsyth, M.; MacFarlane, D. R. J. Mater. Sci. 2003, 38, 3293.

(28) Efthimiadis, J.; Pas, S. J.; Forsyth, M.; MacFarlane, D. R. Solid State Ionics 2002, $154,279$.

(29) Every, H. A.; Bishop, A. G.; MacFarlane, D. R.; Oradd, G.; Forsyth, M. J. Mater. Chem. 2001, 11, 3031.

(30) Ferry, A.; Edman, L.; Forsyth, M.; MacFarlane, D. R.; Sun, J. Z. Electrochim. Acta 2000, 45, 1237.

(31) Jin, L. Y.; Nairn, K. M.; Forsyth, C. M.; Seeber, A. J.; MacFarlane, D. R.; Howlett, P. C.; Forsyth, M.; Pringle, J. M. J. Am. Chem. Soc. 2012, 134, 9688.

(32) Dill, K. A.; Bromberg, S. Molecular Driving Forces: Statistical Thermodynamics in Chemistry and Biology; Garland Science: New York, 2003.

(33) Huang, J. H.; Hill, A.; Forsyth, M.; MacFarlane, D.; Hollenkamp, A. Solid State Ionics 2006, 177, 2569.

(34) Long, S.; MacFarlane, D. R.; Forsyth, M. Solid State Ionics 2003, 161, 105.

(35) Pas, S. J.; Huang, J.; Forsyth, M.; MacFarlane, D. R.; Hill, A. J. J. Chem. Phys.

2005, 122.

(36) Mehrer, H. Diffusion in Solids, 2007; Vol. 155.

(37) Wagner, C.; Schottky, W. Z. Physik. Chem. B 1931, 11, 163.

(38) Baughman, R. H.; Turnbull, D. J. Phys. Chem. Solids 1971, 32, 1375. 
(39) Forsyth, M.; MacFarlane, D. R.; Golding, J. J.; Huang, J.; Sun, J. Chem. Mater. 2002, 14, 2103.

(40) Dosseh, G.; Fressigne, C.; Fuchs, A. H. J. Phys. Chem. Solids 1992, 53, 203

(41) Rana, U. A.; Vijayaraghavan, R.; MacFarlane, D. R.; Forsyth, M. J. Mater. Chem. 2012, 22, 2965.

(42) Abouimrane, A.; Whitfield, P. S.; Niketic, S.; Davidson, I. J. J. Power Sources 2007, 174, 883.

(43) Rana, U. A.; Bayley, P. M.; Vijayaraghavan, R.; Howlett, P.; MacFarlane, D. R.; Forsyth, M. Phys. Chem. Chem. Phys. 2010, 12, 11291.

(44) Rana, U. A.; Vijayaraghavan, R.; MacFarlane, D. R.; Forsyth, M. Chem. Commun. 2011, 47, 6401.

(45) Einstein, A. Ann. Physik 1905, 17, 549.

(46) Edward, J. T. J. Chem. Educ. 1970, 47, 261.

(47) Hou, J.; Li, J.; Madsen, L. A. Macromolecules 2010, 43, 347.

(48) Callaghan, P. T. Translational Dynamics Magnetic Resonance: Principles of Pulsed Gradient Spin Echo NMR, 2011.

(49) Jennifer M. Pringle, P. C. H., Douglas R. MacFarlane and Maria Forsyth J. Mater. Chem. 2010, 20, 2056.

(50) Wilmer, D.; Funke, K.; Witschas, M.; Banhatti, R. D.; Jansen, M.; Korus, G.; Fitter, J.; Lechner, R. E. Physica. B 1999, 266, 60.

(51) Cahill, L. S.; Rana, U. A.; Forsyth, M.; Smith, M. E. Phys. Chem. Chem. Phys. 2010, 12, 5431. 


\section{Chapter 2: Nuclear Magnetic Resonance Theory and Methods}

\subsection{Basic Nuclear Magnetic Resonance (NMR) Theory}

Nuclear magnetic resonance (NMR) is one of the most powerful spectroscopic tools available. Almost every element has an NMR-active isotope and is identifiable through the intrinsic property spin. ${ }^{1,2}$ Typical nuclei studied are ${ }^{1} \mathrm{H},{ }^{31} \mathrm{P},{ }^{13} \mathrm{C}$, and ${ }^{15} \mathrm{~N}$, which are all spin- $-1 / 2$ nuclei and thus are magnetically convenient. NMR active nuclei are classified by their spin quantum number $I$, as presented in Statement 2.1, where $I$ is the spin quantum number: ${ }^{1}$

$$
(2 I+1)
$$

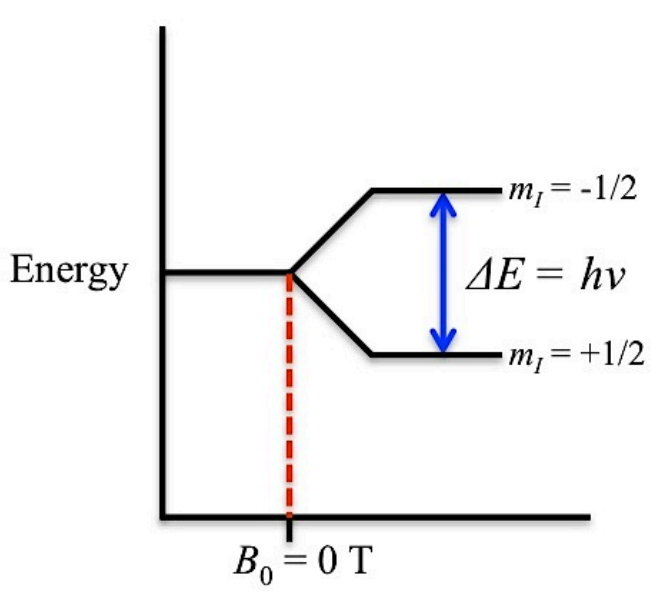

Figure 2.1: In the presence of an externally applied magnetic field, the nuclear spin states of NMR active nuclei are no longer degenerate and thus split, commonly referred to as Zeeman splitting. Shown is an illustration of Zeeman splitting for a typical spin- $1 / 2$ nucleus such as ${ }^{1} \mathrm{H}$ where there are $(2 I+1)$ spin states (i.e., two spin states). ${ }^{1}$ 
These selected nuclei are spin- $1 / 2$, meaning the spin states are two-fold degenerate in zero magnetic field. ${ }^{2}$ In the presence of a magnetic field, the degeneracy is broken resulting in the nuclear spin states splitting, known as the nuclear Zeeman effect (Zeeman splitting) (Figure 2.1). ${ }^{1}$ The magnetic moments of nuclei with a non-zero spin precess around the applied magnetic field direction $(z)$ at the Larmor frequency $\omega_{o}$ as described by Equation $2.1 ; \gamma$ is the gyromagnetic ratio and $B_{0}$ is the strength of the magnetic field. ${ }^{1,2}$

$$
\omega_{o}=-\gamma B_{0}
$$

In the presence of $B_{0}$, the wandering motions of the spin polarizations will result in the buildup of longitudinal magnetization, defined as longitudinal relaxation or spin-lattice relaxation,

commonly characterized by the time constant $T_{1} \cdot{ }^{1,2}$ The other process that can occur in NMR is the relaxation, or de-phasing, of the net magnetization in the transverse plane (x,y-plane), defined as spin-spin relaxation, commonly characterized by the time constant $T_{2} \cdot{ }^{1,2}$ Knowing $T_{1}$ and $T_{2}$ is critical for advanced NMR techniques such as the PGSTE pulse sequence to obtain self-diffusion coefficients, as discussed in section 2.3.

\subsection{General Remarks on Solid-State NMR}

Chemical shift anisotropy (CSA), quadrupole coupling (Q), and dipole-dipole coupling (DD) are the nuclear spin interactions that affect solid-state NMR signals due to their dependence on orientation with respect to the applied magnetic field $B_{0} \cdot{ }^{3-6}$ Analysis of spectral lineshapes affords the study of molecular dynamics occurring in the $\mathrm{kHz}$ frequency range. ${ }^{7}$ Powder samples that contain crystallites with various orientations (i.e., polycrystalline materials) 
can be observed with solid-state NMR. These interactions produce spectral frequencies pertaining to their specific orientations with respect to $B_{0}$ that superimpose to make up a socalled "powder pattern", ${ }^{6}$ which will be discussed in the following section. The OIPC studied for this Thesis was obtained deuterated, ${ }^{8}$ thus we will focus on the fundamentals of ${ }^{2} \mathrm{H}$ NMR since quadrupolar couplings are the most dominant interactions we observe for ${ }^{2} \mathrm{H}$ NMR experiments.

\subsection{1 ${ }^{2}$ H NMR Theory}

For a given sample, the nuclei experience magnetic and electric fields that originate from that sample. ${ }^{6}$ Of the internal spin Hamiltonian terms corresponding to those interactions, the quadrupolar coupling for a solid (Equation 2.2) will only be considered here because all other interactions in ${ }^{2} \mathrm{H}$ NMR of OIPCs are small in comparison. ${ }^{1} \theta_{Q}$ is the angle between the internuclear long axis of a molecular bond containing a quadrupolar nucleus, with spin $I$, and $B_{0}{ }^{1}$

$$
\widehat{H}_{Q}(\theta)=\omega_{Q}\left(\theta_{Q}\right) \times \frac{1}{6}\left(3 \hat{I}_{z}^{2}-I(I+1) \hat{1}\right)
$$

Quadrupolar couplings represent the electric quadrupole interactions of spin $>1 / 2$ nuclei with the surrounding electric field. ${ }^{6}$ Because deuterium has a nuclear spin of 1 , the non-spherical electron clouds confined to the $\mathrm{C}^{2} \mathrm{H}$ bond generate an electric field gradient that interacts with the electric quadrupole moment of the ${ }^{2} \mathrm{H}$ nucleus. The quadrupolar coupling constant $C_{Q}$ (Equation 2.3) defines this interaction:

$$
C_{Q}=\frac{e^{2} q Q}{h}
$$


where $e^{2} q$ is the electric field gradient term, $Q$ is the quadrupole moment, and $h$ is Planck's constant. In a typical aliphatic or aromatic $\mathrm{C}^{2} \mathrm{H}$ bond containing molecule, $C_{Q}$ for ${ }^{2} \mathrm{H}$ is $\sim 180$ $\mathrm{kHz}$; however, in the presence of internal molecular motions the quadrupolar coupling can be motionally averaged, and thus reduced considerably. ${ }^{1}$

$$
\Delta v_{Q}\left(\theta_{Q}\right)=3 \pi C_{Q} \times \frac{1}{2}\left(3 \cos ^{2} \theta_{Q}-1\right)
$$

The present study focuses on solid samples, which consists of randomly oriented $\mathrm{C}_{-}{ }^{2} \mathrm{H}$ bonds with respect to $B_{0} . \Delta v_{Q}$ represents the change in frequency and is dependent on the orientation of the $\mathrm{C}^{2} \mathrm{H}$ bond electric field gradient principal tensor component with respect to $B_{0}$. This angular dependence is described with the aid of the second Legendre polynomial term ${ }^{5}$ in Equation 2.4 and Figure 2.2. 

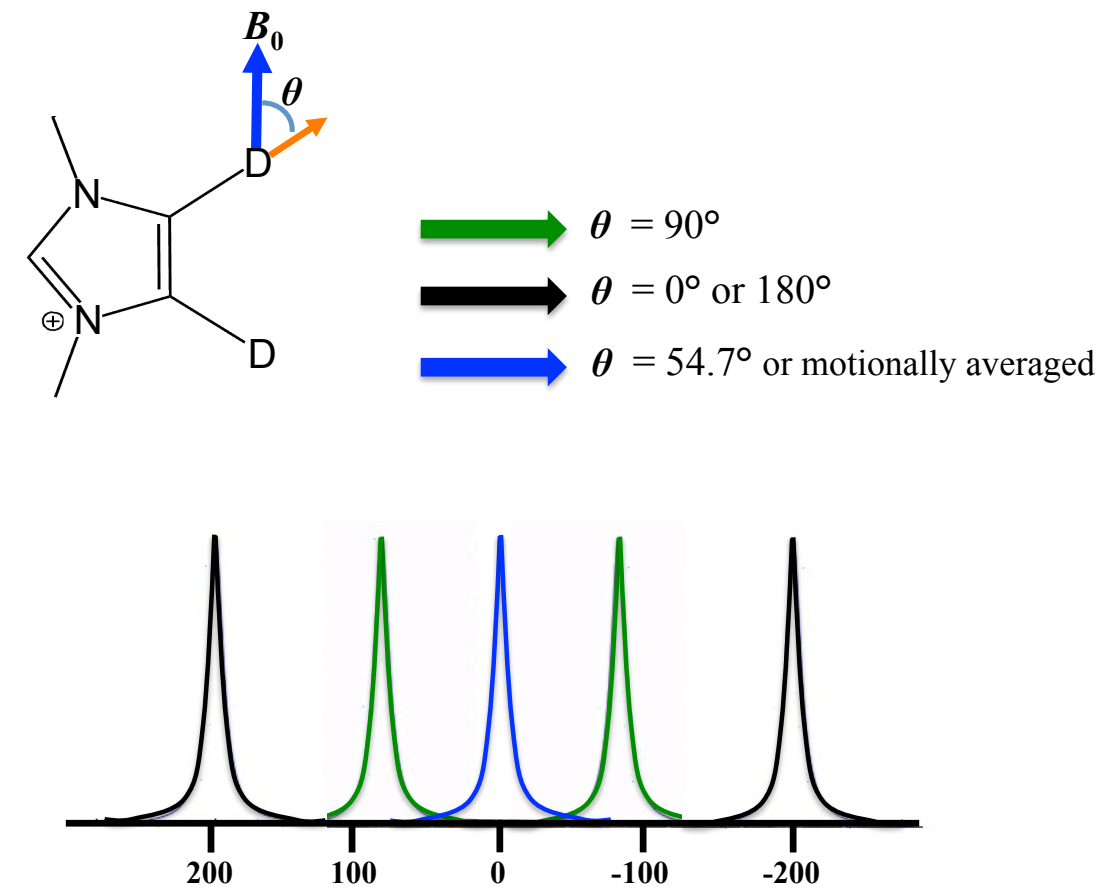

Frequency (kHz)

Figure 2.2: Illustration of $\Delta v_{Q}$ as a function of $\theta$ for an isotropic material. The angle dependence can be described by the second Legendre polynomial term in Equation 2.4.

Since the isotopically labeled compound of interest is a polycrystalline material, one would expect to observe a distribution of first-order splitting's, $\Delta v_{Q}$, displaying a powder pattern; also known as a Pake doublet (Figure 2.3). The common case of a uniaxial electric field gradient tensor $(E F G T)\left(\eta_{\mathrm{Q}}=0\right)$ produces such a spectrum. The powder pattern is a superposition of every possible molecular orientation that produces its own chemical shift. ${ }^{1}$ The larger signals come from the molecules whose principal axis of the EFGT is perpendicular to the magnetic field, $\theta=90^{\circ}$. The weakest signals come from the molecules whose unique principal axis of the 
EFGT is parallel to the magnetic field, $\theta=0^{\circ}$ and $/$ or $180^{\circ}{ }^{1}$ The quadrupolar spin-echo pulse sequence (Figure 2.4) is used to refocus the ${ }^{2} \mathrm{H}$ magnetization. ${ }^{2}$

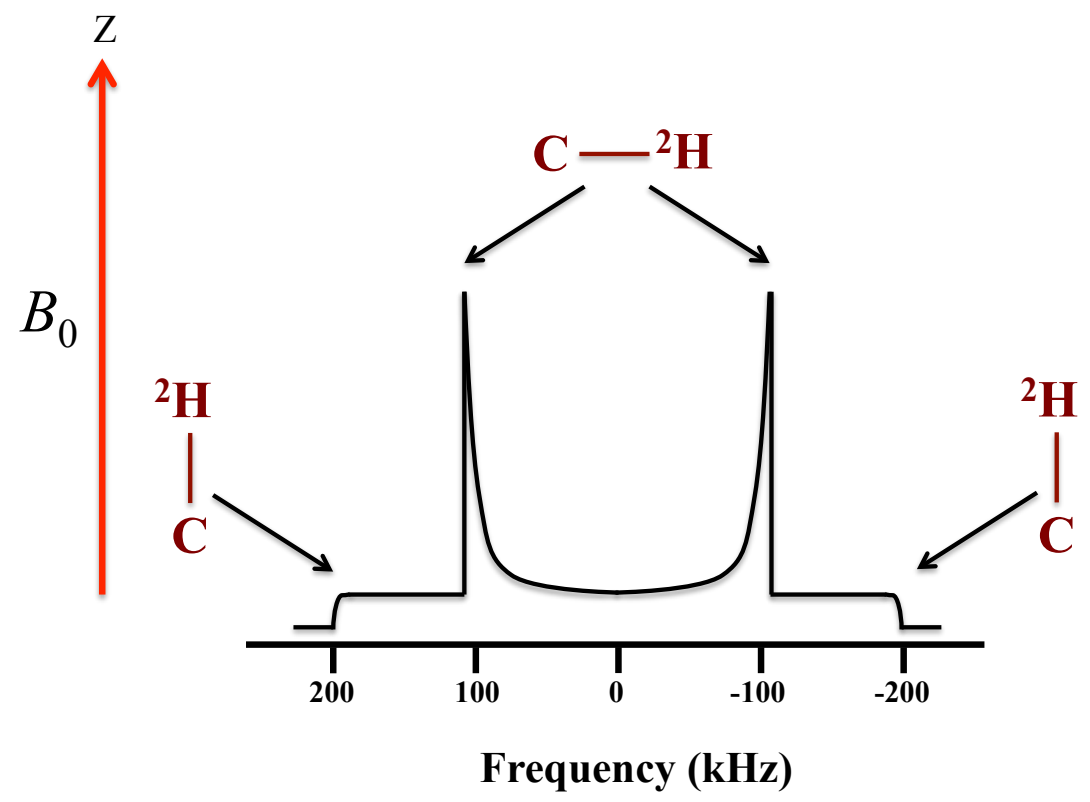

Figure 2.3: A distribution of first-order splittings produces the well known Pake doublet; where most of the signal intensity comes from the $\mathrm{C}-{ }^{2} \mathrm{H}$ bonds perpendicular, $\theta=90^{\circ}$, to the magnetic field. ${ }^{1}$

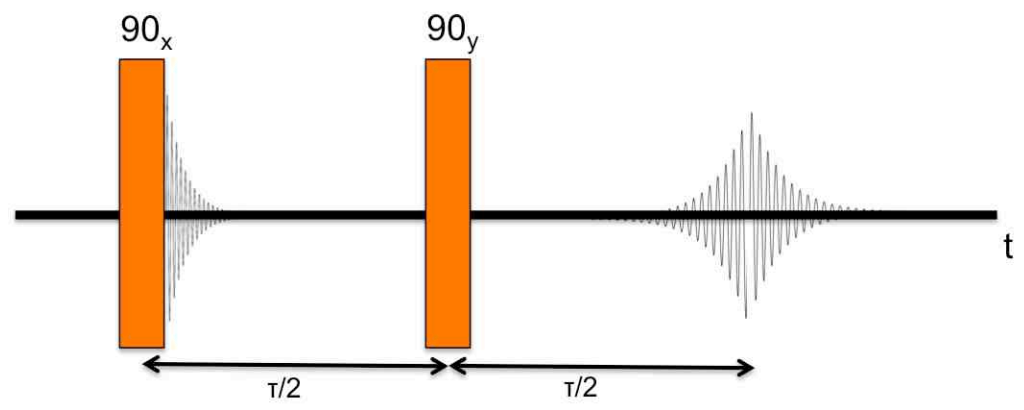

Figure 2.4: The quadrupolar spin-echo pulse sequence is commonly used in solid materials to refocus ${ }^{2} \mathrm{H}$ magnetization that relaxes upon excitation of the $90_{\mathrm{y}}{ }^{\circ}$ pulse. ${ }^{2}$ 
A change in $\Delta v_{Q}$ will be seen as the sample goes from the anisotropic solid-state to the isotropic liquid-state. Meaning, the crystallites will go from a static random molecular orientation with respect to $B_{0}$ to an isotropic state. As the system is heated over time, a change in the shape of the powder pattern will be seen as $T_{\mathrm{m}}$ of the material is reached. The crystallites will begin moving and the $\Delta v_{Q}$ will decrease as temperature increases. This is due to the molecules no longer being confined to static orientations with respect to $B_{0}$ and becoming motionally averaged (Figure 2.5).

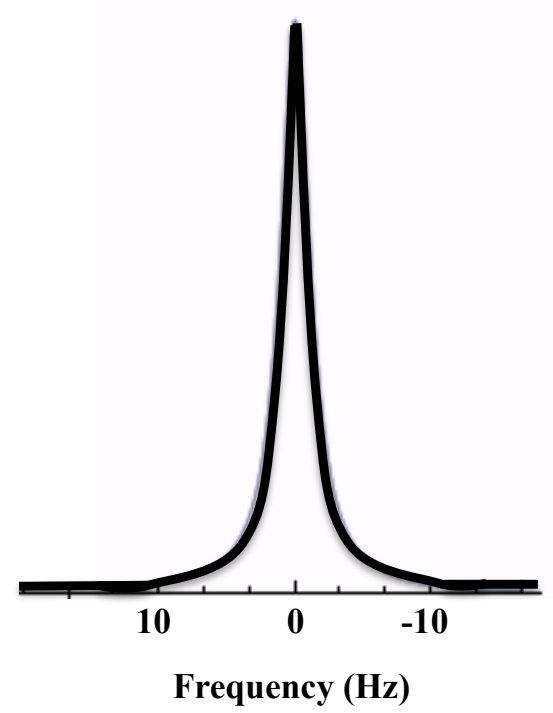

Figure 2.5: Illustration of isotropic motional averaging occurring above $T_{m}$.

With the goal of understanding ion transport within the high temperature solid phases of OIPCs, a high temperature environment must be employed. As previously mentioned, linebroadening effects, such as CSA, DD, and Q couplings can make it difficult to properly understand specific molecular environments. However, isotopic labeling (i.e., replacing ${ }^{1} \mathrm{H}$ with $\left.{ }^{2} \mathrm{H}\right)$ allows access to very specific molecular environments that other techniques do not offer, as will be shown in the Chapter 3 . 


\subsection{Pulsed-Field-Gradient NMR}

Thus far it has only been mentioned that the self-diffusion coefficient of a molecular species proves to be a powerful value while also providing insight to the molecular environment encompassing the diffusing species. In the world of NMR, the heart of this phenomenon lies in

magnetic field inhomogeneity. ${ }^{9}$ Magnetic field inhomogeneity causes signal broadening as a result of small deviations in $\omega_{0}$ (Larmor frequency) as a result of an imperfect magnetic field $B_{0}$, as described by Equation 2.1. ${ }^{9}$ Modern day super-conducting magnets are equipped with multiple-order shim coils, which serve to manipulate the magnetic field within and around a given sample to produce as homogenous of a magnetic field as possible, as spectral resolution is ultimately dependent on $B_{0}$ homogeneity. ${ }^{9}$

In 1950, Erwin Hahn made one of the most important discoveries in the evolution of NMR, the formation of a spin echo. ${ }^{10}$ Almost all modern-day NMR techniques would not be possible without this phenomenon. Upon excitation with a $90^{\circ} \mathrm{rf}$ pulse, the net magnetization dephases (i.e., "fans out") in the transverse plane (x,y-plane), thus leading to an inherent signal attenuation. As mentioned earlier, the fundamental relaxation process that describes this phenomenon is spin-spin relaxation, commonly referred to as $T_{2}$. After some time period $\tau$, prior to full signal attenuation, a $180^{\circ} \mathrm{rf}$ pulse is applied such that the net magnetization is "flipped" and refocused, thus forming a "spin echo". However, Hahn found that once the magnetization was flipped, incomplete refocusing of the net magnetization lead to an "echo attenuation". Realizing there was something else underlying the signal attenuation other than purely $T_{2}$, Hahn suggested that the echo attenuation was partially due to the diffusion of molecules in the presence of magnetic field inhomogeneity. ${ }^{10}$ The elucidation and quantification of the selfdiffusion coefficient would later be proposed by Carr and Purcell ${ }^{11}$ in 1954 and further 
exemplified by Stejskal and Tanner in $1965,{ }^{12}$ who ultimately finalized the mathematical relationship of the time-dependent diffusion with the observed NMR signal (discussed below).

Revisiting Equation 2.1, we showed that a nucleus with a spin-quantum number $I \geq 1 / 2$ has an intrinsic resonance frequency $\omega_{0}$ in the presence of an externally applied magnetic field $B_{0}$. If we assume $B_{0}$ is homogenous at all locations along the z-axis, then $\omega_{0}$ for each spin is the same at all locations. Adding a magnetic field gradient $\mathbf{g}\left(\mathrm{G} \mathrm{cm}^{-1}\right)$ (Equation 2.5), where $\mathbf{i}, \mathbf{j}$, and $\mathbf{k}$ are unit vectors: ${ }^{13}$

$$
\mathbf{g}=\nabla B_{0}=\frac{\partial B_{Z}}{\partial x} \mathbf{i}+\frac{\partial B_{Z}}{\partial y} \mathbf{j}+\frac{\partial B_{Z}}{\partial z} \mathbf{k}
$$

to the already existing $B_{0}$ causes $\omega_{0}$ to become dependent on the overall strength of the magnetic field at varying locations, thus producing a linear variation in $\omega_{0}$ along the sample (Equation 2.6). ${ }^{13}$

$$
\omega_{0}=\gamma\left(B_{0}+\mathrm{g} z\right)=\omega_{0}+\gamma \mathrm{g} z
$$

This allows us to spatially encode spins by linearly varying the magnetic field with a homogenous magnetic field gradient. ${ }^{13}$ Figure 2.6 is an illustration of how the magnetization helix evolves under the influence of gradient pulses and a simple spin echo pulse sequence. Each magnetization vector, or "spin isochromat", is in phase upon excitation of a $90^{\circ} \mathrm{rf}$ pulse in the transverse plane. The introduction of a gradient, with strength g, causes the spin isochromats to wind and form a magnetization helix with wavelength $\lambda$ (Equation 2.7):

$$
\lambda=\frac{2 \pi}{\gamma \mathrm{g} \delta}
$$


wherein $\gamma$ is the gyromagnetic ratio of the nucleus and $\delta$ is time over which the gradient is applied.
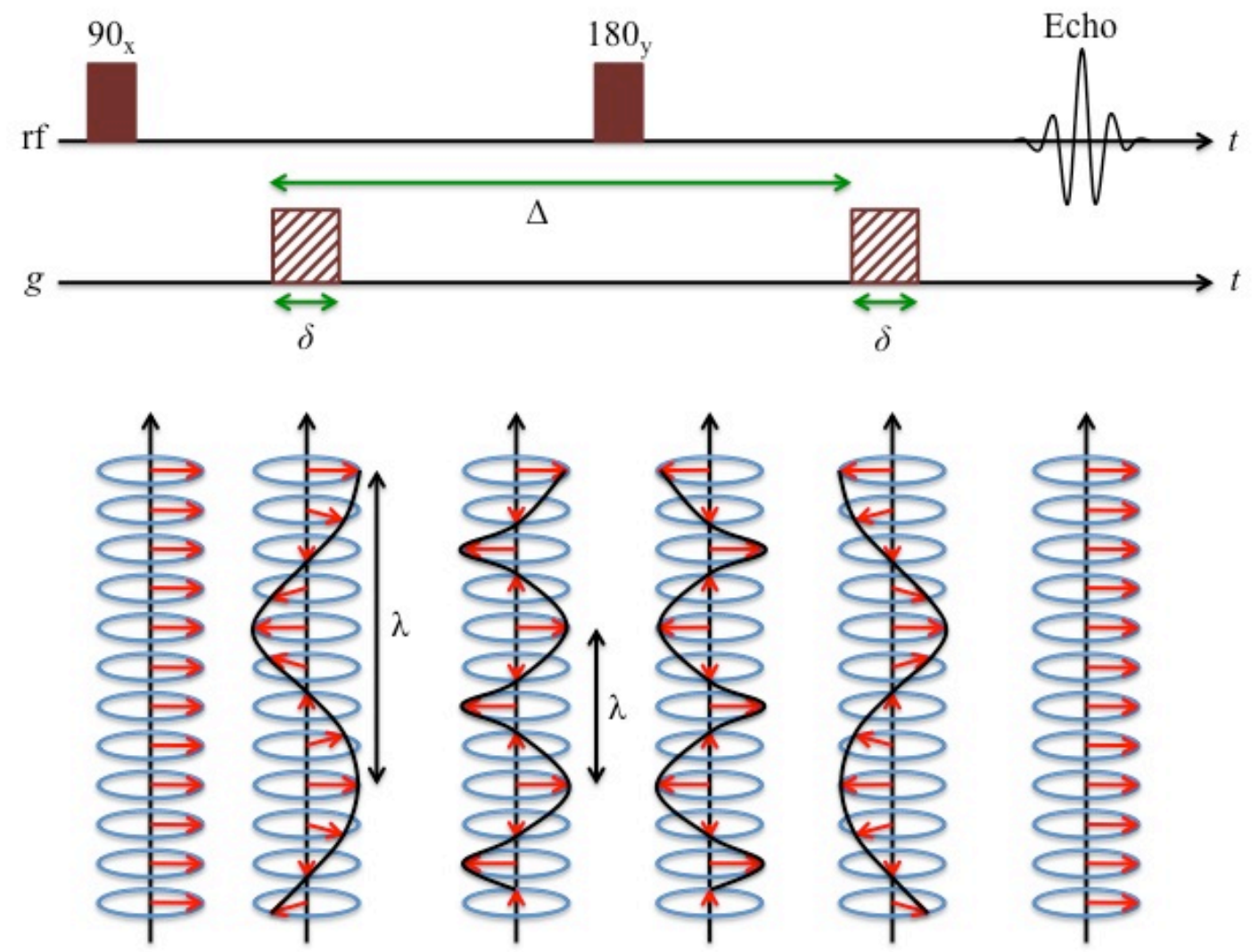

Figure 2.6: A simple spin echo pulse sequence in the presence of a pair of matched externally applied magnetic field gradients is commonly used to determine the diffusion coefficient by linearly varying the strength of an externally applied magnetic field gradient. A $90^{\circ} \mathrm{rf}$ pulse causes all spin isochromats to be in phase within the transverse plane (x,y-plane). Upon the application of a gradient, the spin isochromats form a "magnetization helix" with wavelength $\lambda$. A $180^{\circ}$ rf pulse flips the helix accompanied by another gradient pulse (after the encoding time $\Delta$ ) unwinds the magnetization helix, thus forming a spin echo. Figure reproduced from Callaghan. ${ }^{9}$ 
In Figure 2.6, it can be seen that the wavelength of the helix decreases with time as a result of the spin isochromats becoming more de-phased in the transverse plane. A $180^{\circ} \mathrm{rf}$ pulse flips the spin isochromats where they are eventually unwound and refocused with another a matched gradient pulse, thus forming the spin echo. A phase shift $\varphi$ in the refocused net magnetization occurs due to a change in spin position along the axis the gradient is applied during the encoding time $\Delta$ (Equation 2.8): ${ }^{9}$

$$
\varphi=\gamma g \delta\left(z_{1}-z_{2}\right)
$$

There is a strong dependence on the gradient strength $\mathrm{g}$, with $\gamma$ and $\delta$ constant. A mathematical relationship to describe the signal attenuation as a function of gradient strength did not arise until Stejskal and Tanner developed what is now referred to as the Stejskal-Tanner equation (Equation $2.9):^{12}$

$$
I=I_{0} e^{-D \gamma^{2} \mathrm{~g}^{2} \delta^{2}\left(\Delta-\frac{\delta}{3}\right)}
$$

wherein $I$ is the signal intensity, $\gamma$ is the gyromagnetic ratio, $\mathrm{g}$ is the gradient strength, $\delta$ is the effective gradient pulse length, $\Delta$ is the diffusion time, and $D$ is the self-diffusion coefficient. ${ }^{12}$ The effective gradient pulse length, $\delta$, and the diffusion time, $\Delta$, are dependent on the relaxation time constants $T_{2}$ and $T_{1}$, respectively. The self-diffusion coefficient is determined by linearly varying the externally applied magnetic field gradient across the sample, such that the signal intensity exponentially decreases as a function of gradient strength (Figure 2.7) and fit to the Stejskal-Tanner equation (Equation 2.9). 


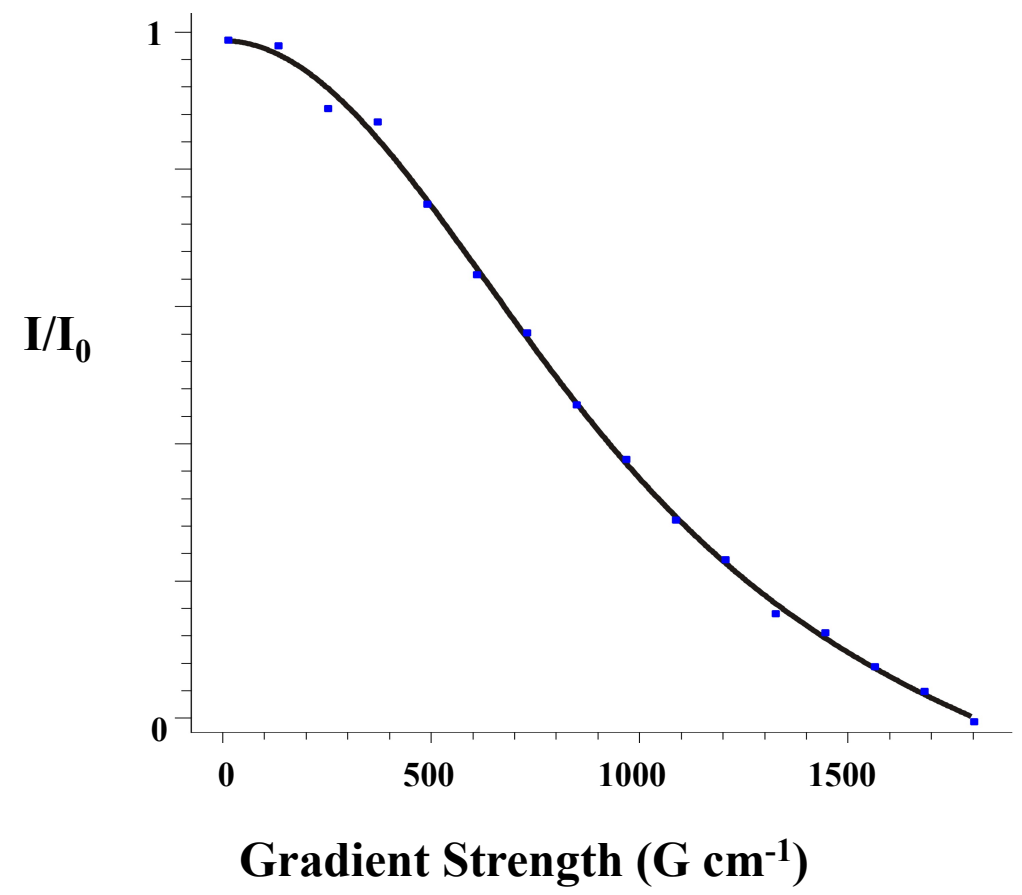

Figure 2.7: A gaussian decay in signal intensity $I$ as a function of gradient strength $g$ is observed when carrying out the PFG-NMR experiment. The self-diffusion coefficient is extracted by fitting with the Stejskal-Tanner equation (Equation 2.9). ${ }^{12}$

For a polycrystalline material, the crystallites are in a rigid environment and thus the nuclei within are not motionally averaged. Upon rf excitation, nuclei relaxation in the transverse plane (i.e., $T_{2}$ ) will occur much more rapidly (ms - $\mu$ s time-scale) versus nuclei in a liquid. This causes the signal intensity to attenuate much faster than can be acquired. Typically, the $T_{2}$ relaxation time constant is the limiting factor for the pulsed-field gradient stimulated echo (PGSTE) experiment as it defines the effective gradient pulse length that can be used. The PGSTE pulse sequence is sufficient for $T_{2} \geq 3 \mathrm{~ms}$. It should be noted that PFG-NMR studies are 
$T_{2}$-weighted. That is, signals that have short $T_{2}$ 's $(\sim<3 \mathrm{~ms})$ will not be detected, as the spin-spin relaxation occurs more rapidly than acquisition.

Described in detail by Stejskal and Tanner, pulsed-field-gradient NMR (PFG-NMR) represents a powerful tool that gives direct insight into the molecular diffusion within a system. ${ }^{12}$ We can extend this view to cation and anion transport in OIPCs, allowing a deeper understanding of the diffusion mechanism for each. PFG-NMR is defined by the principles of molecular diffusion, as discussed in Chapter 1, whereby molecular diffusion is the random translational (Brownian) motion of particles. $^{2}$ Ion transport in a polycrystalline material can occur through multiple mechanisms, such as ion hopping through the crystalline lattice and grain boundary diffusion. Variable temperature PFG-NMR can report on individual cation and anion $E_{\mathrm{a}}$ values in the many solid phases typically observed for OIPCs. Knowing such values is critical for this study, as the activation energy can help describe the transport mechanism of diffusive ions, leading to a deeper understanding of OIPC inter- and intra-molecular interactions. Understanding $D, E_{\text {a }}$, and $D_{0}$ can help determine if that species is diffusing through the crystalline lattice via vacancies (i.e., Schottky-disorder) or via grain boundaries and give a deeper understanding of the molecular environment. This is especially beneficial since conductivity measurements are bulk measurements and thus it is impossible to distinguish which ion is dominating conductivity. As will be shown in Chapter 3, factors such as thermal treatment and the application of an externally applied magnetic field potentially change $E_{\mathrm{a}}$ for diffusing species, suggesting a change in transport mechanism.

\section{References:}

(1) Levitt, M. H. Spin Dynamics, 2008; Vol. 2.

(2) Roberts, J. D. $A B C^{\prime}$ 's of FT-NMR, 2000. 
(3) Reichert, D. Prog. Nucl. Magn. Reson. Spectrosc. 2005, 47, 137.

(4) Ashbrook, S. E.; Duer, M. J. Concept. Magn. Reson. 2006, 28A, 183.

(5) Duer, M. J. Introduction to Solid-State NMR Spectroscopy: Principles and Applications, 2002; Vol. 1.

Vol. 2.

(6) Duer, M. J. Solid-State NMR Spectroscopy Principles and Applications, 2004;

(7) Dixon, W. T. J. Magn. Reson. 1984, 57, 85.

(8) Lee, M.; Choi, U. H.; Wi, S.; Slebodnick, C.; Colby, R. H.; Gibson, H. W. J. Mater. Chem. 2011, 21, 12280.

(9) Callaghan, P. T. Translational Dynamics Magnetic Resonance: Principles of Pulsed Gradient Spin Echo NMR, 2011.

(10) Hahn, E. Phys. Rev. 1950, 80, 580.

(11) Carr, H.; Purcell, E. Phys. Rev. 1954, 94, 630.

(12) E. Stejskal, J. T. J. Chem. Phys. 1965, 42.

(13) Price, W. S. Concepts Magn. Reson. 1997, 5, 299. 


\title{
Chapter 3
}

\section{Cation and Anion Transport in a Dicationic}

\section{Imidazolium-Based Plastic Crystal Ion Conductor}

\author{
Bryce E. Kidd, Mark D. Lingwood, Minjae Lee, Harry W. Gibson, and Louis A. Madsen*
}

Department of Chemistry and Macromolecules and Interfaces Institute

Virginia Polytechnic Institute and State University, Blacksburg, VA 24061

Key words: organic ionic plastic crystal, grain boundary, NMR, self-diffusion, activation energy, Stokes-Einstein relation.

This chapter will soon be submitted for publication in Journal of Physical Chemistry B, hence the manuscript presentation format. Bryce Kidd conceived and executed the data acquired, Dr. Mark Lingwood aided in the guidance of efficient data acquisition and interpretation, Dr. Minjae Lee synthesized the material, and Dr. Harry W. Gibson and Dr. Louis Madsen provided deep discussions on understanding the data. 
Abstract: Here we investigate the organic ionic plastic crystal (OIPC) 1,2-bis[ $N-\left(N^{\prime}-\right.$ hexylimidazolium- $\left.\left.d_{2}(4,5)\right)\right] \mathrm{C}_{2} \mathrm{H}_{4} 2 \mathrm{PF}_{6}^{-}$in one of its solid plastic crystal phases by means of multi-nuclear solid-state (SS) NMR and pulsed-field-gradient (PFG) NMR. We quantify distinct cation and anion diffusion coefficients as well as the diffusion activation energies $\left(E_{\mathrm{a}}\right)$ in this dicationic imidazolium-based OIPC. Our studies suggest a change in transport mechanism for the cation upon varying thermal and magnetic treatment $(9.4 \mathrm{~T})$, evidenced by changes in cation and anion $E_{\mathrm{a}}$. Moreover, variable temperature ${ }^{2} \mathrm{H}$ SSNMR lineshapes support a change in local molecular environment upon slow cooling in $B_{0}$. Additionally, we quantify the percentage of mobile anions as a function of temperature from variable temperature ${ }^{19} \mathrm{~F}$ SSNMR, wherein two distinct spectral features are present. We also comment on the pre-exponential factor $\left(D_{0}\right)$, giving insight into the number of degrees of freedom for both cation and anion as a function of thermal treatment. In conjunction with previously reported conductivity values for this class of OIPCs and the Stokes-Einstein relation, we propose that ion conduction is dominated by anion diffusion between crystallites (i.e., grain boundaries). Using our experimentally determine diffusion coefficient and previously reported $\mathrm{PF}_{6}^{-}$hydrodynamic radius $\left(r_{H}\right)$, viscous $(\eta=4.11 \mathrm{~Pa} \cdot \mathrm{s})$ ionic liquid (IL) is present with a cation $r_{H}$ of $0.343 \mathrm{~nm}$. NMR measurements are very powerful in elucidating fundamental OIPC properties and allow a deeper understanding of ion transport within such materials. 


\subsection{Introduction}

Solid-state electrolytes, such as the perfluorosulfonate ionomer Nafion ${ }^{\circledR}$, have garnered much interest in the past decades for use in applications ranging from batteries to fuel cells. Not only have new applications emerged, but also new chemistry within this complex system has been unveiled. From a battery and fuel cell standpoint, a highly conductive static matrix that allows sufficient charge transport from anode to cathode is required, and this conduction arises from intrinsic properties of the solid-state electrolyte. While ionic polymers such as Nafion ${ }^{\circledR}$ are exceptional solid-state electrolytes, ion conduction is highly dependent on small volatile molecules (usually water) to mediate charge transport. This dependence on a volatile small molecule charge carrier results in reduction of conductivity at elevated temperatures when the small molecule evaporates out of the electrolyte matrix.

In contrast, a relatively new class of solid-state electrolytes, organic ionic plastic crystals (OIPCs), have shown competitive ion conductivity $\left(10^{-3} \mathrm{~S} \mathrm{~cm}^{-1}\right)$ without the disadvantages of polymer electrolyte conductors. Similar to ionic liquids (ILs), OIPCs consist purely of cations and anions. At room temperature $\left(25^{\circ} \mathrm{C}\right)$, an IL is a liquid salt, while an OIPC is a solid salt. ${ }^{1}$ Unlike ILs, OIPCs exhibit efficient packing through long-range crystalline order of ions, but have short-range disorder where isotropic states (i.e., isotropic rotational mobility of the cation and/or anion about one or more axes) exist below the melting temperature $\left(T_{m}\right) \cdot{ }^{1-16}$ OIPCs have generated great interest due to high conductivity in one or more solid phases, which is attributed to joint rotational and translational motions within the crystalline lattice (i.e., rotator phase). Many classes of OIPCs possess multiple high enthalpy first-order solid-solid phase transitions before $T_{m}$ and conductivity tends to increase in the higher temperature phases. ${ }^{1,15-17}$ 
It is well known that ion conduction within an ionic solid can occur through the classical "ion hopping" mechanism, whereby open vacancies within the crystalline lattice promote diffusion. If vacancies are present, charge neutrality must be conserved; thus for every vacant cation, there must be a vacant anion within the lattice (commonly known as Schottky disorder). In conjunction with conductivity and DSC, positron annihilation spectroscopy (PALS) studies on OIPCs have been successful in showing relationships between the size and number of vacancies as a function of temperature, where discontinuous changes in the free-volume of vacancies are observed. Such behavior supports the ion hopping mechanism, as strong evidence suggests correlations between sizes of individual cations/anions, cation-anion pairs, and free-volume changes within the crystalline lattice. While this holds true for OIPCs composed of small cations and/or anions, large steric or ionic potential barriers in the crystalline lattice can make ion transport difficult for more bulky cations and anions. For a system where fast ion conduction is present but cannot reasonably occur in the crystalline lattice, one must rethink the ion transport mechanism.

Because OIPCs are polycrystalline materials, grain boundaries on the nanometer-tomicron scale are naturally present. In general, grain boundaries form as a result of a mismatch in crystallite orientations in a polycrystalline material. Methods such as synthesis, crystallite nucleation when cooling from above $T_{\mathrm{m}}$, thermal treatment, and small-applied forces can induce and control the size and number of grain boundaries present in a polycrystalline material. Representing large geometric restrictions, grain boundaries act as a "sink" for non-crystalline molecules, as the lowest free energy state is the crystallite. Interestingly, previous work by Dosseh et al. showed that molecular plastic crystals, such as 1,4 -dioxane $\left(T_{m}=11{ }^{\circ} \mathrm{C}\right)$, cyclohexanol $\left(T_{m}=25{ }^{\circ} \mathrm{C}\right)$, cyclohexane $\left(T_{m}=7{ }^{\circ} \mathrm{C}\right)$, pivalic acid $\left(T_{m}=37{ }^{\circ} \mathrm{C}\right)$, and 
succinonitrile $\left(T_{m}=58{ }^{\circ} \mathrm{C}\right)$, melt at the grain boundary below $T_{m}$. This is commonly known as the pre-melting phenomenon and occurs at the surface of a crystallite where gradual melting prior to $T_{\mathrm{m}}$ will occur. Through variable temperature ${ }^{1} \mathrm{H}$ SSNMR, Dosseh et al. observed a twocomponent system with a narrow signal centered on top of a broad signal. The narrow and broad signals were suggested to be due to material in the grain boundary and crystallite, respectively. Approaching $T_{m}$ causes the broad signal to diminish, accompanied by an increase in narrow signal. For OIPCs, similar distinct NMR spectral features have been reported for pure and doped forms of the materials. However, such spectral features are highly dependent on the cation-anion pair and concentration of dopant.

In the Nernst-Einstein equation: ${ }^{12}$

$$
\sigma=\frac{\left(D_{+} c_{+}+D_{-} c_{-}\right) q^{2}}{k T}
$$

$\sigma$ is conductivity, $D_{+/-}$are diffusion coefficients for cation $(+)$and anion $(-), c_{+/-}$are concentrations of each charge carrier, $q$ is the ionic charge dominating conductivity, and $k T$ is the average thermal energy ( $k$ is Boltzmann's constant and $T$ is temperature). It can be seen that there is a strong dependence on $D_{+/-}$and $c_{+/-}$with $\sigma$. Since grain boundaries do not represent the lowest free energy state, a small concentration of non-crystalline cations and anions may reside within the grain boundary with an intrinsically small diffusion coefficient, thus resulting in low conductivity. Diffusion through the grain boundary represents a new and interesting way to think about how ion transport within polycrystalline materials (and other materials) occurs, where diffusion and local energetics may not follow an "ion hopping" mechanism. The Arrhenius equation:

$$
D=D_{0} e^{\frac{-E_{a}}{k T}}
$$


affords the temperature $T$ dependent diffusion coefficient $D$, thus giving insight into local energetics (i.e., activation energy $E_{\mathrm{a}}$ ) on the pre-diffusion $(<1 \mathrm{~nm})$ length scale (commonly referred to as the inertial/ballistic motion length scale); wherein $D_{0}$ is classically known as diffusion at infinite temperature and $k T$ is the average thermal energy. A deeper investigation on the pre-diffusion length scale is imperative in understanding how collective interactions govern the transport of small molecules in various environments, particularly environments where a crystalline lattice does not exist but diffusion is prevalent. Since $E_{\text {a }}$ gives insight into the prediffusion local energetics, short time scale ion pairs (dipoles), triples, and quadrupoles can contribute to the measured diffusion coefficient. This can affect the extracted $E_{\mathrm{a}}$, as it is dependent on $D$, which is also dependent on the above mentioned short time scale interactions. Interestingly, $D_{0}$ can be used to report on the configurational degrees of freedom (i.e., entropic contributions) within a system, thus giving insight into the local environment encompassing the diffusing species.

While Timmerman's criterion, conductivity, PALS, and X-ray diffraction have been used extensively to understand the many OIPCs studied thus far, it is apparent that chemically specific transport properties, such as the self-diffusion coefficient $D$ and activation energy $E_{\mathrm{a}}$, will bring more comprehensive understanding of ion transport and local energetics within such systems. Other techniques of measuring transport, e.g., conductivity measurements, rely on bulk measurements and fail to report the behavior of individual cations and anions. Ion transport within OIPCs is possible due to the nature of crystalline imperfections present, such as lattice vacancies and grain boundaries; thus a technique that can separately quantify cation and anion contributions to ion conduction is necessary. ${ }^{18-20}$ 
Here we employ multi-nuclear pulsed-field-gradient (PFG) NMR to report on prediffusional local energetics (i.e., $E_{\mathrm{a}}$ ) and multi-nuclear 1D NMR to spectroscopically observe crystalline and non-crystalline ions. We show that the measured ion transport and local energetics of the cation and anion occur within the grain boundary, thus giving insight into previously reported conductivity values for this class of OIPCs. More interestingly, cooling in a strong magnetic field $\left(B_{0}=9.4 \mathrm{~T}\right)$ induces a broad distribution of crystallite domain alignments such that $E_{\mathrm{a}}$ for the cation increases from 14 to $39 \mathrm{~kJ} \mathrm{~mol}^{-1}$. To our knowledge, we also present the first reported magnetic realignment in an OIPC, whereby the cation shows drastic changes in $E_{\mathrm{a}}$ with thermal and magnetic treatment while the anion does not. Additionally, we quantify the percentage of mobile anions as a function of temperature from variable temperature ${ }^{19} \mathrm{~F}$ SSNMR, wherein two distinct spectral features are present. We also comment on the pre-exponential factor $\left(D_{0}\right)$, giving insight into the configurational degrees of freedom ${ }^{21}$ and how the local environment affects $E_{\mathrm{a}}$ for both cation and anion as a function of thermal treatment.

\subsection{Experimental}

\subsubsection{Sample Preparation}

The deuterated analog of bis[N-(N'-hexylimidazolium $)]$ salt was synthesized in three steps (Scheme 1):

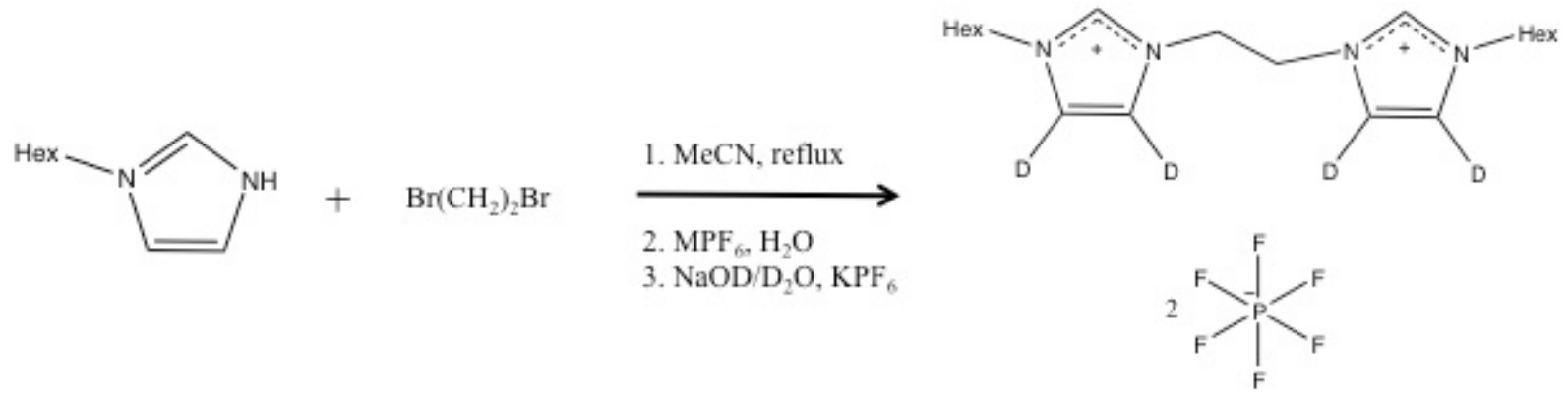

Scheme 1: Synthesis of 1,2-bis $\left[N-\left(N^{\prime}\right.\right.$-hexylimidazolium- $\left.\left.d_{2}(4,5)\right)\right] \mathrm{C}_{2} \mathrm{H}_{4} 2 \mathrm{PF}_{6}^{-}$. 
the quaternization coupling reaction of 1-hexylimidazole (2 molar equivalents) with dibromoalkane (1 molar equivalent) followed by $\mathrm{PF}_{6}{ }^{-}$exchange in water. Deuterium exchange on the 4- and 5-positions of the imidazolium was done with $\mathrm{NaOD}$ in $\mathrm{D}_{2} \mathrm{O}$. The final product was precipitated in aqueous $\left(\mathrm{H}_{2} \mathrm{O}\right)$ saturated $\mathrm{KPF}_{6}$ solution and was a white crystalline solid at room temperature $\left(25^{\circ} \mathrm{C}\right)$. It should be noted that the 2-position was also deuterated but reverted back to protonated upon introduction of the saturated $\mathrm{KPF}_{6}$ solution. ${ }^{15,16}$ The material $(\sim 30 \mathrm{mg})$ was loaded into a $5 \mathrm{~mm}$ outer diameter glass bulb, heated in an oil bath under vacuum to $210^{\circ} \mathrm{C}\left(T_{\mathrm{m}}=\right.$ $\left.196{ }^{\circ} \mathrm{C}\right)$, and de-gassed to prevent degradation. Upon cooling to room temperature $\left(25^{\circ} \mathrm{C}\right)$ and pumping overnight, the glass bulb was sealed with an oxygen/natural gas torch.

\subsubsection{Variable Temperature Solid-State NMR}

All ${ }^{1} \mathrm{H} /{ }^{2} \mathrm{H} /{ }^{19} \mathrm{~F}$ variable temperature SSNMR experiments were performed using a 400 MHz Bruker Avance III WB NMR spectrometer, equipped with a wideline static solids probe. The sample was allowed to equilibrate at each temperature for twenty minutes prior to spectral acquisition. The quadrupolar echo pulse sequence ${ }^{22}$ (Figure 2.6) was used to acquire ${ }^{2} \mathrm{H}$ NMR spectra and a simple pulse-acquire sequence was used to acquire ${ }^{1} \mathrm{H} /{ }^{19} \mathrm{~F}$ NMR spectra. For this study, a series of deuterated standards, hexamethylbenzene- $d_{18}\left(\mathrm{HMB}-d_{18}\right)\left(T_{m}=\sim 167{ }^{\circ} \mathrm{C}\right)$ purchased from Sigma Aldrich with $>99 \%$ purity, urea- $d_{4}\left(T_{m}=\sim 133{ }^{\circ} \mathrm{C}\right)$ purchased from Sigma Aldrich with $>98 \%$ purity, and the liquid crystal 2,5-bis-( $p$-hydroxyphenyl)-1,3,4-oxadiazole (ODBP-Ph-C 7 -d6) (phase map: crystal to smectic $\mathrm{Y}\left(148{ }^{\circ} \mathrm{C}\right)$, smectic $\mathrm{Y}$ to $\mathrm{B} 2\left(166{ }^{\circ} \mathrm{C}\right)$, B2 to biaxial nematic $\left(173{ }^{\circ} \mathrm{C}\right)$, biaxial nematic to uniaxial nematic $\left(215^{\circ} \mathrm{C}\right)$, and uniaxial nematic to isotropic $\left.\left(222^{\circ} \mathrm{C}\right)\right)^{23,24}$ were used to calibrate the probe at multiple temperatures, as each of these 
materials display distinct changes in quadrupole splitting $\Delta v_{\mathrm{Q}}$ at their respective phase transitions.

\subsubsection{Variable Temperature Pulsed-Field-Gradient NMR}

All variable temperature ${ }^{1} \mathrm{H}$ and ${ }^{19} \mathrm{~F}$ PFG-NMR experiments were performed using a 400 $\mathrm{MHz}$ Bruker Avance III WB NMR spectrometer, equipped with an MIC probe coupled to a Diff60 single-axis (z-axis) gradient system. The pulsed-gradient stimulated echo (PGSTE) sequence (Figure 2.8) was used with a maximum gradient strength of $1850 \mathrm{G} \mathrm{cm}^{-1}$, an effective gradient pulse length of $\delta=2 \mathrm{~ms}$, and a diffusion time of $\Delta=35 \mathrm{~ms}$.

\subsection{Results and Discussion}

As seen in Figure 3.1, the phase map for the present OIPC displays three solid-solid phase transitions, which all critically impact the ion transport due to variances in phase behavior. ${ }^{15,16}$ High $T_{\mathrm{m}}$ 's for this class of OIPCs are due to the many hydrogen - fluorine contacts, reported from single crystal X-ray diffraction. ${ }^{16}$ For the present study, our variable temperature multi-nuclear NMR experiments probed phases I through $\mathrm{S}_{3}$ and our variable temperature multinuclear PFG-NMR experiments probed phase $\mathrm{S}_{3}$, due to probe temperature limitations.

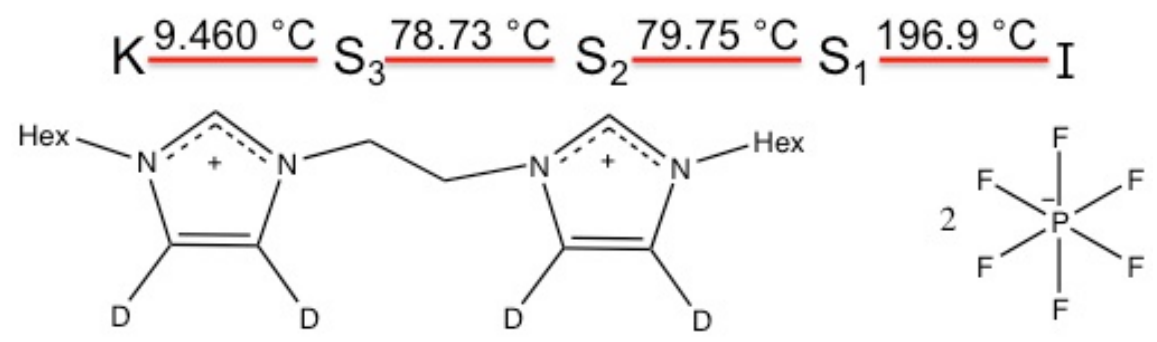

Figure 3.1: Chemical structure and phase map of the ionic compound 1,2-bis $\left[N-\left(N^{\prime}-\right.\right.$ hexylimidazolium- $\left.\left.d_{2}(4,5)\right)\right] \mathrm{C}_{2} \mathrm{H}_{4} 2 \mathrm{PF}_{6}^{-}$used in this study. The 4 and 5 positions of the 
imidazolium ring were deuterated, as previously reported. ${ }^{15,16} \mathrm{~K}, \mathrm{~S}_{3}, \mathrm{~S}_{2}$, and $\mathrm{S}_{1}$, all represent solid phases and I represents the isotropic melt.

\subsection{1 ${ }^{2}$ H NMR vs. Temperature}

Figure 3.2 shows a representative ${ }^{2} \mathrm{H}$ NMR spectrum obtained at room temperature (25 ${ }^{\circ} \mathrm{C}$, phase $\mathrm{S}_{3}$ ), which displays a superposition of doublets (i.e., a single doublet represents a crystallite at a specific angle $\theta$ to $B_{0}$ ), thus forming the well know Pake powder pattern, ${ }^{25}$ indicating that the crystallites have random orientations with respect to $B_{0}$. Such a spectrum can be described by the second Legendre polynomial term $\frac{1}{2}\left(3 \cos ^{2} \theta_{Q}-1\right)$ in the quadrupole splitting equation:

$$
\Delta v_{Q}\left(\theta_{Q}\right)=3 \pi C_{Q} \times \frac{1}{2}\left(3 \cos ^{2} \theta_{Q}-1\right)
$$

wherein $\theta$ is the angle of the $\mathrm{C}-{ }^{2} \mathrm{H}$ bond $\left({ }^{2} \mathrm{H}\right.$ being a spin $\mathrm{I}=1$ quadrupolar nucleus) with respect to the $B_{0}$ and $C_{Q}$ is the quadrupole coupling constant $\left(\sim 168 \mathrm{kHz}^{15}\right){ }^{26}$ The Pake powder pattern forms as a result of the random distribution of $\mathrm{C}-{ }^{2} \mathrm{H}$ bond angles with respect to $B_{0}$ for a polycrystalline material. Upon further inspection of Figure 3.2, there is a small amount of material that produces a single peak, centered at $0 \mathrm{kHz}$. This small isotropic signal likely arises from motionally averaged $\mathrm{C}-{ }^{2} \mathrm{H}$ angles on a small population of cation molecules in a liquid-like state. $^{25}$ 


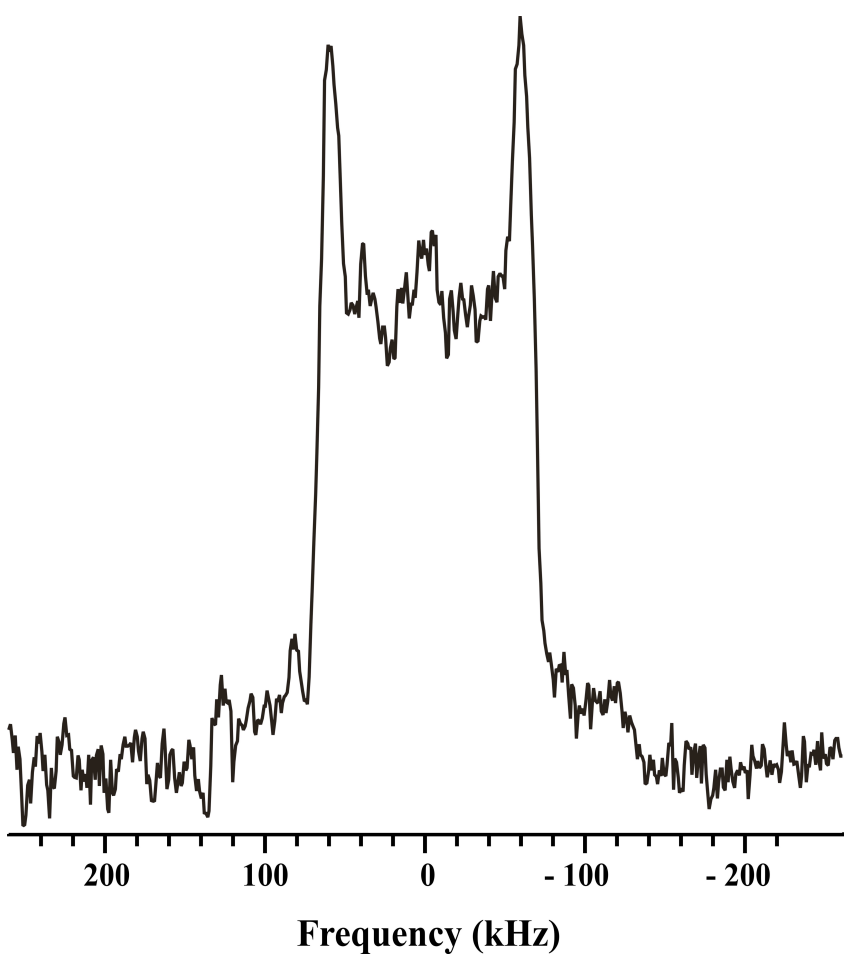

Figure 3.2: Room temperature $\left(25^{\circ} \mathrm{C}\right){ }^{2} \mathrm{H}$ NMR measurement of the OIPC 1,2-bis[ $[N-(N$ 'hexylimidazolium- $\left.\left.d_{2}(4,5)\right)\right] \mathrm{C}_{2} \mathrm{H}_{4} 2 \mathrm{PF}_{6}{ }^{-}$displays the well-known Pake powder pattern, confirming the random orientation of crystallites with respect to $B_{0}$. A $\Delta v_{\mathrm{Q}}$ value of $\sim 121 \mathrm{kHz}$ is observed.

We studied the temperature dependence of ${ }^{2} \mathrm{H}$ NMR to probe the imidazolium dynamics within the solid phases. Figure 3.3 shows ${ }^{2} \mathrm{H}$ NMR stacked spectra as a function of temperature, specifically in phases $\mathrm{I}, \mathrm{S}_{1}$, and $\mathrm{S}_{3}$. At $T_{m}\left(196^{\circ} \mathrm{C}\right)$ a single narrow signal is observed, indicating that the quadrupolar interactions average to zero due to rapid tumbling of molecules in the isotropic (melt) state. Upon cooling to $85^{\circ} \mathrm{C}$ (phase $\mathrm{S}_{1}$ ), some fraction of the isotropic phase still exists, suggesting that isotropic tumbling of the cations occurs, even in the solid state. At $50{ }^{\circ} \mathrm{C}$ (phase $\mathrm{S}_{3}$ ), two distinct spectral features, a narrow signal residing on a broad signal, are observed. However, at $30{ }^{\circ} \mathrm{C}$ (phase $\mathrm{S}_{3}$ ) the isotropic signal diminishes dramatically along with the broad shoulders, leaving behind a broad doublet due to an anisotropic distribution of 
crystallite structures along $B_{0}$. This variable temperature ${ }^{2} \mathrm{H}$ NMR result suggests that melting and then cooling within the magnetic field induces alignment of crystalline domains inside this material.

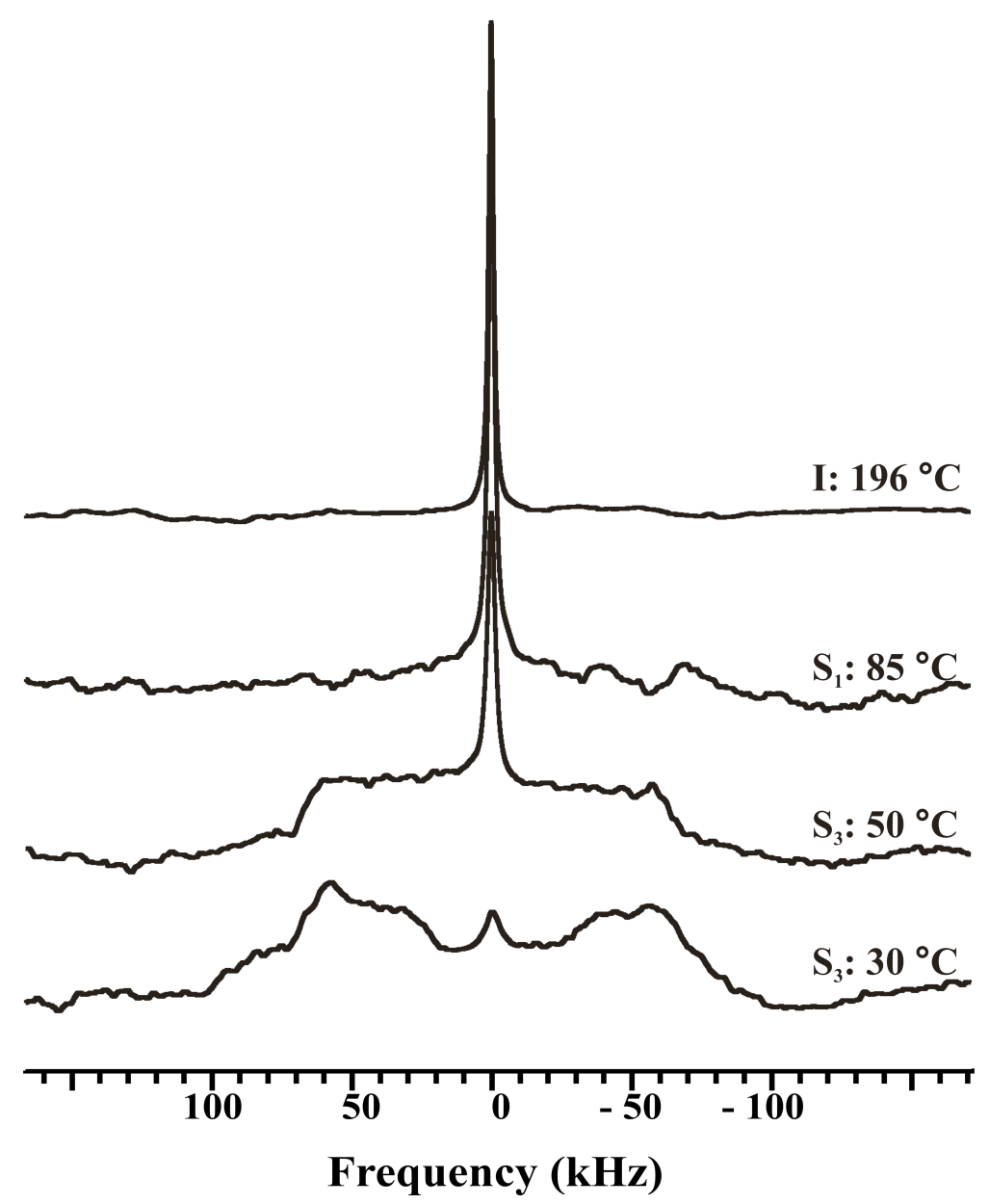

Figure 3.3: ${ }^{2} \mathrm{H}$ NMR spectra vs. temperature displaying imidazolium orientation and dynamics in phases $\mathrm{I}, \mathrm{S}_{1}$, and $\mathrm{S}_{3}$. One observes weak alignment upon cooling in the magnetic field, evidenced by the broad quadrupolar doublet in phase $\mathrm{S}_{3}$ at $30^{\circ} \mathrm{C}$.

We can propose that there is a broadly distributed but anisotropic crystallite alignment upon melting and subsequent cooling in the presence of $B_{0}$. These ${ }^{2} \mathrm{H}$ results are the first of their kind, not only for variable temperature ${ }^{2} \mathrm{H}$ NMR on the present OIPC, but also in observing 
induced alignment of an OIPC in the presence of a strong magnetic field. Figure 3.4 gives a simple illustration to depict realignment of the crystallites upon cooling in $B_{0}$. The implication of orientation control of an OIPC, by inducing alignment with a strong magnetic field, opens a new avenue of research for OIPCs. By isotopically labeling the material (i.e., replacing ${ }^{1} \mathrm{H}$ with ${ }^{2} \mathrm{H}$ ), orientational dependence with respect to $B_{0}$ can be probed.

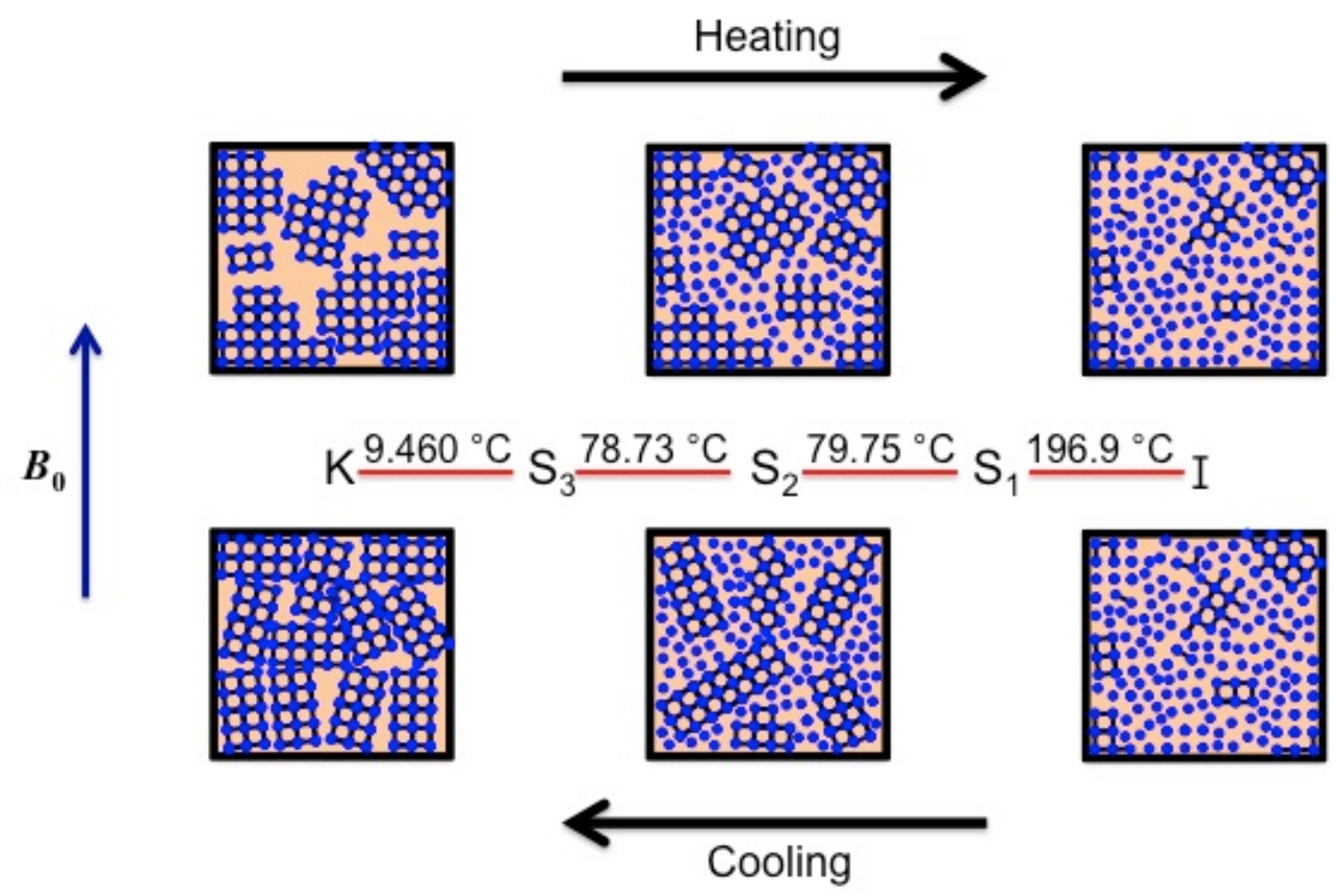

Figure 3.4: Illustration of potential crystallite realignment in the presence of a magnetic field upon heating to phase I and cooling to phase $\mathrm{S}_{3}$. Cooling in $B_{0}$ affords the formation of anisotropic crystallites such that the crystallite long-axis orientation is biased along $B_{0}$. This is possible due to the presence of mobile species within the grain boundary, thus allowing the crystallites to move and form some weak ordering. 
The remaining isotropic signal present in Figures 3.2 and 3.3 suggests that cations exist in a second environment (i.e., grain boundary), other than in the crystallite. Recall that Figure 3.2 contains a single peak that can be attributed to restricted imidazolium rotations about the C-N bonds from crystalline cations and/or cations in the grain boundary isotropically averaged. Considering the crystal structure (Figure 1.12), ${ }^{16}$ and the possibility of $\mathrm{PF}_{6}^{-}$crystalline vacancies, one could envisage the imidazolium rings to be less sterically hindered and exhibit restricted rotation about the C-N bonds. Recently, Lee et al. conducted variable temperature $\left(0-100{ }^{\circ} \mathrm{C}\right)$ magic-angle spinning ${ }^{2} \mathrm{H}$ NMR; it was suggested that the imidazolium rotational dynamics do not change across phases $S_{3}$ and $S_{2}$, evidenced by no significant spectral changes. ${ }^{15}$ If noncrystalline cations reside in a second environment (e.g., grain boundary) where steric hindrance is much less, a narrower signal associated with the isotropic molecular tumbling can be observed due to the averaging out of the second Legendre polynomial; thus we conclude that the residual ${ }^{2} \mathrm{H}$ signals in Figures 3.2 and 3.3 are from grain boundary cations.

\subsection{2 ${ }^{19}$ F and ${ }^{1} H$ NMR vs. Temperature}

To augment our variable temperature ${ }^{2} \mathrm{H}$ NMR results, we are also investigating how the anion $\left({ }^{19} \mathrm{~F}\right)$ and remaining cation structure $\left({ }^{1} \mathrm{H}\right)$ dynamics change as a function of temperature in the solid phases, as this gives a better understanding of the material as a whole (i.e., does the cation or anion contribute to the conductivity). Figure 3.5 shows representative ${ }^{19} \mathrm{~F}$ NMR spectra as a function of temperature $\left(205-40^{\circ} \mathrm{C}\right)$ in $20^{\circ}$ increments below the isotropic spectrum. In the isotropic phase we clearly observe the $J_{\mathrm{PF}}$ coupling (i.e., doublet in Figure 3.5) between phosphorous and fluorine. The coupling constant is $J_{\mathrm{PF}}=\sim 720 \mathrm{~Hz}$, which is the same coupling constant observed in a standard solution of $\mathrm{KPF}_{6}$. Cooling the sample to phase $\mathrm{S}_{1}$ drastically decreases the intensity of the isotropic signal accompanied by a broad signal, centered about the 
isotropic signal. Such spectral features have been observed for other OIPCs ${ }^{6,14,27}$ and molecular crystals $;{ }^{28}$ the isotropic signal was sometimes suggested to be ions diffusing in the grain boundary, while the broad signal was suggested to be ions in a rigid environment, such as a crystallite. This leads us to suggest that the isotropic signal in Figure 3.5 arises from $\mathrm{PF}_{6}{ }^{-}$within the grain boundary while the broad signal is crystalline $\mathrm{PF}_{6}^{-}$. Cooling from phase $\mathrm{S}_{1}$ to $\mathrm{S}_{3}$ gradually decreases the isotropic signal, indicative of a decrease in the number of mobile $\mathrm{PF}_{6}{ }^{-}$ ions in the grain boundary. This is an indication that while the material is well below its melting point, there is still isotropic averaging of $\mathrm{PF}_{6}^{-}$within the temperature range studied. We might be tempted to rationalize the isotropic averaging observed from the variable temperature ${ }^{19} \mathrm{~F}$ NMR by invoking the octahedral geometry of $\mathrm{PF}_{6}{ }^{-}$and the crystal structure (Figure 1.12) ${ }^{16}$ wherein the rotation of $\mathrm{PF}_{6}{ }^{-}$about the fixed crystalline lattice point is allowed due to the nature of the unit cell, as discussed in Chapter 1. However, we clearly see two spectral components in Figure 3.5, where the broad signal is due to $\mathrm{PF}_{6}^{-}$in a relatively rigid or slowly rotating environment (in crystallites), while the narrow component is due to $\mathrm{PF}_{6}^{-}$in a rapidly rotating environment (in non-crystalline grain boundaries). 


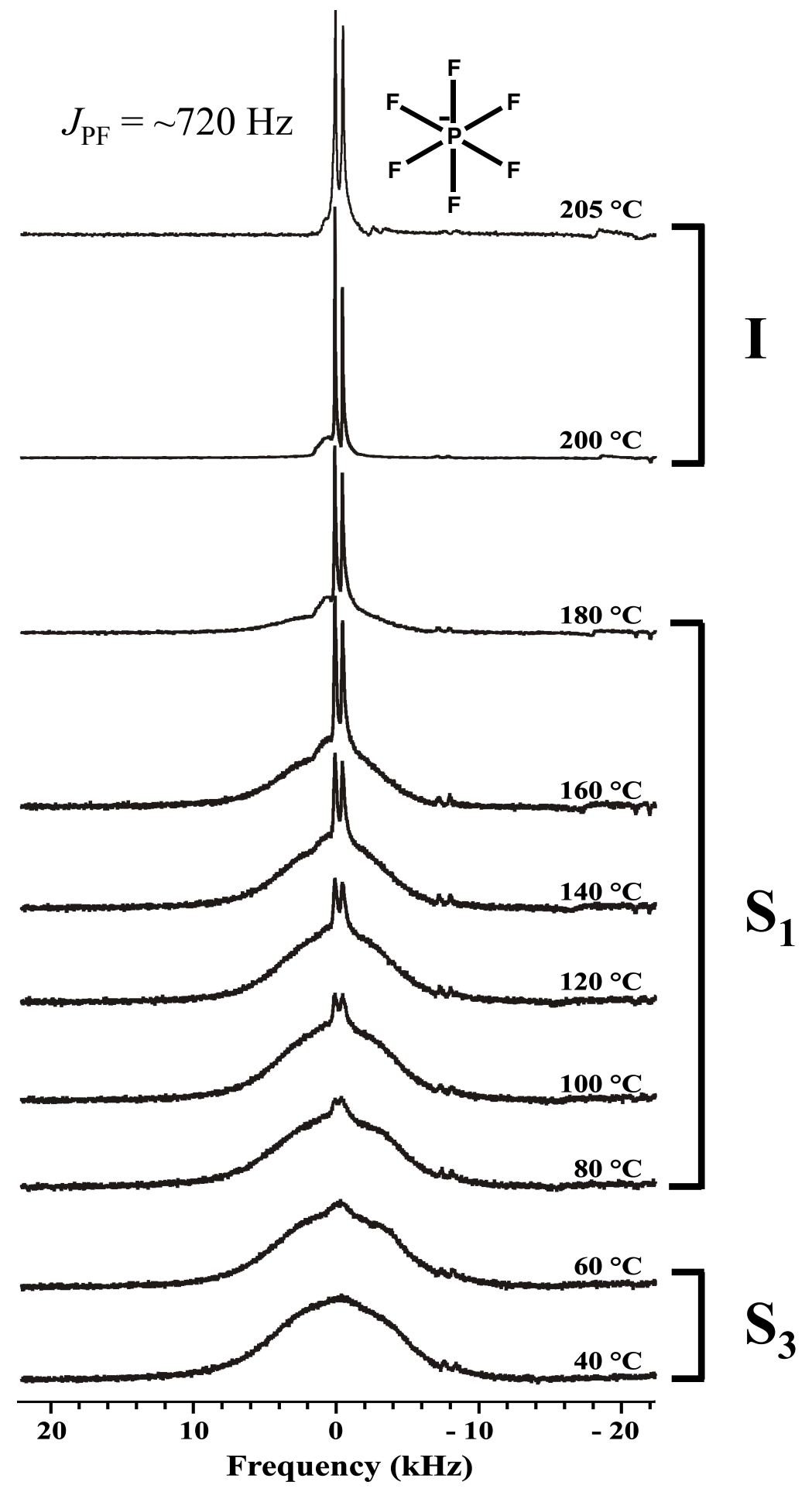

Figure 3.5: ${ }^{19} \mathrm{~F}$ spectra as a function of temperature. Spectra at $205^{\circ} \mathrm{C}, 200{ }^{\circ} \mathrm{C}$, and $180{ }^{\circ} \mathrm{C}$ are decreased in vertical scale by a factor of 10,10 , and 4 , respectively, relative to the other spectra. An isotropic signal is visible from phase I to phase $\mathrm{S}_{3}$ with $J_{\mathrm{PF}}=\sim 720 \mathrm{~Hz}$, consistent with the $J$ 
coupling for a standard of $\mathrm{KPF}_{6}$. The isotropic signal suggests fast rotating and diffusive $\mathrm{PF}_{6}{ }^{-}$in the grain boundary, supporting our ${ }^{19} \mathrm{~F}$ PFG-NMR results. The broad signal is due to $\mathrm{PF}_{6}{ }^{-}$in the crystallites.

Quantifying the amount of $\mathrm{PF}_{6}^{-}$in the grain boundary and crystallite from Figure 3.5, through integration of the broad and narrow spectral components, gives a measure of the percentage of $\mathrm{PF}_{6}^{-}$in the grain boundary (Figure 3.6). To compensate for the area integrated below the isotropic signal (i.e., part of the broad signal) a reference area under the broad signal is used to subtract from the isotropic value. At $200{ }^{\circ} \mathrm{C}$ (just below phase I), $\mathrm{PF}_{6}{ }^{-}$in the crystallite exists with $\sim 60 \% \mathrm{PF}_{6}^{-}$isotropically averaged, thus implying that $\sim 60 \%$ of the $\mathrm{PF}_{6}^{-}$signal is from the grain boundaries. With decreasing temperature, an exponential like decrease in isotropic $\mathrm{PF}_{6}^{-}$is observed. Although ionic conductivity measurements for the OIPC used in this study have not been reported, variable temperature $\sigma$ values on the order of $10^{-5}-10^{-14} \mathrm{~S} \mathrm{~cm}^{-1}$ were reported for 1,2-bis $\left[N-\left(N^{\prime}\right.\right.$-nonylimidazolium $\left.)\right] \mathrm{C}_{2} \mathrm{H}_{4} 2 \mathrm{PF}_{6}^{-}, 1,2$-bis $\left[N\right.$-( $\left(N^{\prime}\right.$-decylimidazolium $\left.)\right] \mathrm{C}_{2} \mathrm{H}_{4} 2 \mathrm{PF}_{6}^{-}$, and 1,2-bis $\left[N-\left(N^{\prime}\right.\right.$-dodecylimidazolium $\left.)\right] \mathrm{C}_{2} \mathrm{H}_{4} 2 \mathrm{PF}_{6}{ }^{-15}$. Recall, the Nernst-Einstein equation (Equation 3.1) relates conductivity to the diffusion coefficients and concentrations of charge carrying species. Thus, the small percentage of isotropic $\mathrm{PF}_{6}{ }^{-}$and small diffusion coefficient (discussed below) observed in the large temperature window can explain the initially low and accelerating increase in conductivity with $T$ reported for this class of OIPCs. ${ }^{15}$ Previous work by Dosseh et al. reported on premelting of polycrystalline materials at the surface of grain boundaries prior to $T_{\mathrm{m}} \cdot{ }^{28}$ Based on the present variable temperature ${ }^{19} \mathrm{~F}$ NMR results, it is possible that we are observing gradual melting of the material at the surface of the grain 
boundary until $\sim 150{ }^{\circ} \mathrm{C}$ when a more dramatic increase in "local melting" occurs as the temperature approaches $T_{\mathrm{m}}$.

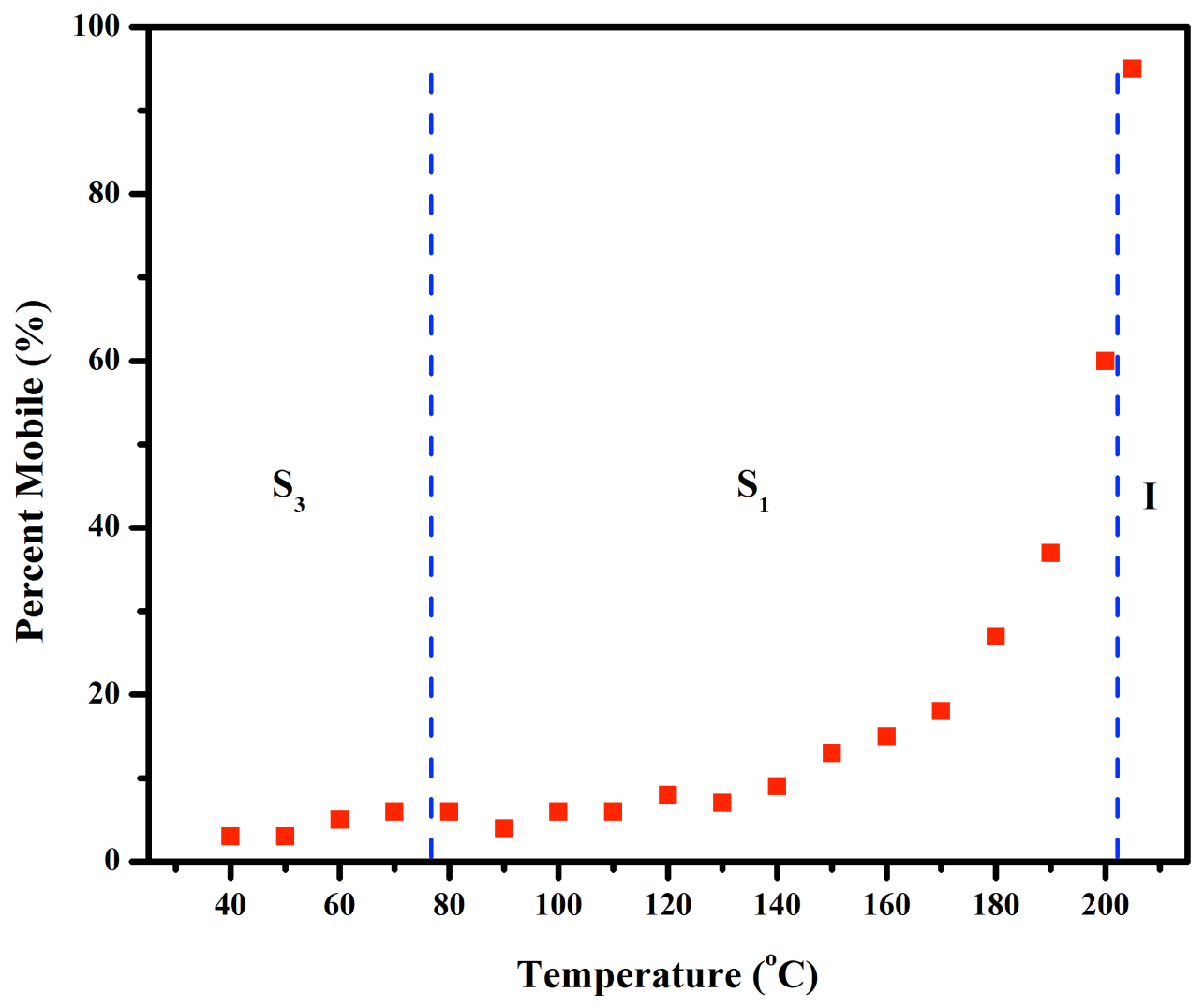

Figure 3.6: Percentage of mobile $\mathrm{PF}_{6}^{-}$(e.g., in the grain boundary) as a function of temperature. We observe a gradual increase in percentage below phase $S_{1}$, and an exponential like growth within phase $\mathrm{S}_{3}$.

We also conducted variable temperature ${ }^{1} \mathrm{H}$ NMR (Figure 3.7). We do not observe the same two-component spectral features as our ${ }^{19} \mathrm{~F}$ NMR results; however, like our ${ }^{2} \mathrm{H}$ NMR results, spectral changes are observed in phase $\mathrm{S}_{3}$. Since ${ }^{1} \mathrm{H}$ is a spin- $1 / 2$ nucleus, we do not obtain the same orientation dependence with respect to $B_{0}$ as ${ }^{2} \mathrm{H}$, which is a spin-1 nucleus, 
further emphasizing the advantage of isotopic labeling. Interestingly, isotropic mobility exists well below $T_{\mathrm{m}}$, which is consistent with our variable temperature ${ }^{2} \mathrm{H}$ NMR spectra, where an isotropic signal is observed even at $85^{\circ} \mathrm{C}$. We conclude that the isotropic signal observed in our ${ }^{2} \mathrm{H}$ measurements is representative of mobile cations within the solid phases of this material. 


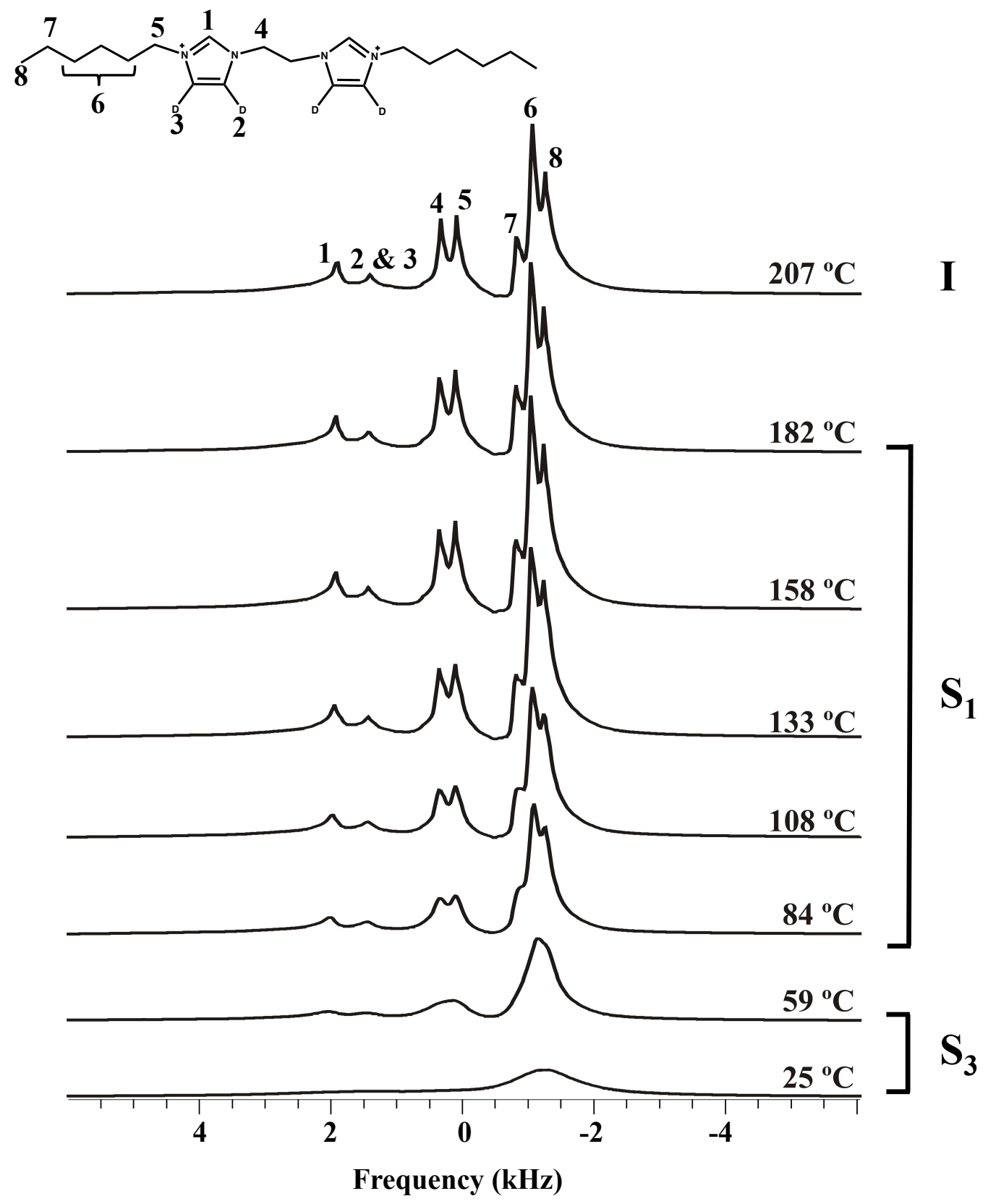

Figure 3.7: ${ }^{1} \mathrm{H}$ NMR spectra as a function of temperature. Above $T_{\mathrm{m}}$, we clearly see all ${ }^{1} \mathrm{H}$ signals. A residual signal from ${ }^{1} \mathrm{H} 2$ and 3 remain after ${ }^{2} \mathrm{H}$ exchange reaction. It should be noted that signals 2 and 3 differ by $52 \mathrm{~Hz}(0.13 \mathrm{ppm}$ at $400 \mathrm{MHz})$ in acetone- $d_{6},{ }^{16}$ thus our signal is too broad to resolve the individual signals. Unlike the variable temperature ${ }^{19} \mathrm{~F}$ spectra, we do not observe two-component spectral features for the variable temperature ${ }^{1} \mathrm{H}$ spectra. We do observe 
a change in spectral features when cooling in phase $S_{3}$, which is consistent with our variable temperature ${ }^{2} \mathrm{H}$ NMR spectral changes in phase $\mathrm{S}_{3}$; however, ${ }^{1} \mathrm{H}$ spectra do not report on the orientation dependence with respect to $B_{0}$, further emphasizing the advantage of isotopic labeling.

\subsubsection{Thermal Treatments}

Previous work by Rana et al. on the OIPC choline dihydrogen phosphate [Choline][DHP] doped with phosphoric acid $\left[\mathrm{H}_{3} \mathrm{PO}_{4}\right]$, trifluoromethanesulfonic acid $[\mathrm{TfOH}]$, and bistrifluoromethanesulfonyl imide $\left[\mathrm{HN}(\mathrm{Tf})_{2}\right]$ showed that the thermal history of an OIPC should be considered. Reported were changes in ${ }^{1} \mathrm{H}$ NMR spectra for the pure material "as-prepared" and annealed (temperature was held at $100{ }^{\circ} \mathrm{C}$ for five days) with no dopant. ${ }^{27}$ The ${ }^{1} \mathrm{H}$ NMR spectra at $100^{\circ} \mathrm{C}$ for both materials display two distinct spectral components, a broad component $(\sim 35$ $\mathrm{kHz})$ representative of crystalline material and a narrow component $(\sim 300 \mathrm{~Hz})$ representative of non-crystalline material. The narrow component was suggested to be slowly translating cations. Annealing the material afforded the formation of less defective crystallites (i.e., less Schottkydisorder) and/or fewer/smaller grain boundaries, as evidenced by the increase in the broad component (i.e., more crystalline material) and decrease in conductivity. The largest increase in conductivity was observed when doped with $4 \mathrm{wt} \%\left[\mathrm{HN}(\mathrm{Tf})_{2}\right]$. Conductivity at the phase III to II transition $\left(21{ }^{\circ} \mathrm{C}\right)$ increased from $\sim 10^{-8}$ to $10^{-5} \mathrm{~S} \mathrm{~cm}^{-1}$ and the conductivity at the phase II to I transition $\left(120^{\circ} \mathrm{C}\right)$ increased from $\sim 10^{-5}$ to $\sim 10^{-3} \mathrm{~S} \mathrm{~cm}^{-1} \cdot{ }^{27}$

To study the effect of varying thermal history on $E_{\mathrm{a}}$, we present a systematic study of ${ }^{1} \mathrm{H}$ and ${ }^{19} \mathrm{~F}$ PFG-NMR experiments with three different thermal treatments (slowly cooled in $B_{0}$ and slowly/rapidly cooled out of $B_{0}$ ) in phase $\mathrm{S}_{3}$. From our variable temperature ${ }^{2} \mathrm{H}$ NMR results, we propose that crystallite realignment occurs upon melting and slow cooling in a $9.4 \mathrm{~T}$ magnetic 
field, which can change the local ion transport mechanism, as compared to material melted and cooled outside of $B_{0}$. The material was melted in an oil bath, removed, and allowed to cool to room temperature, resulting in rapid cooling of the sample and erasing the crystallite alignment history. The final thermal treatment was to melt and slowly cool the material outside of $B_{0}$. The DSC result for the present material was acquired with a temperature-scanning rate of $5 \mathrm{~K} \mathrm{~min}^{-}$

${ }^{1}{ }^{15,16}$ In order to closely emulate the cooling rate, the material was melted in an oil bath and allowed to cool to room temperature in the oil bath, resulting in a slowly cooled thermal treatment.

\subsubsection{Diffusion Coefficient vs. Temperature}

We accomplished cation $\left({ }^{1} \mathrm{H}\right)$ and anion $\left({ }^{19} \mathrm{~F}\right)$ PFG-NMR experiments on the OIPC 1,2$\operatorname{bis}\left[N-\left(N\right.\right.$ '-hexylimidazolium- $\left.\left.d_{2}(4,5)\right)\right] \mathrm{C}_{2} \mathrm{H}_{4} \quad 2 \mathrm{PF}_{6}{ }_{6}$. In a PFG-NMR experiment, we measure signal intensity, $I / I_{0}$, as a function of gradient strength, $g$, and fit to the Stejskal-Tanner equation (Equation 2.7) to extract the self-diffusion coefficient $D .{ }^{29}$ In the PFG experiment, a $T_{2}$-weighted spectrum is produced (Figure 3.8) as a result of $T_{2}$ relaxation during the gradient pulse. Thus, when compared to static spectra, signals with relatively short $T_{2}$ 's are not observed. 


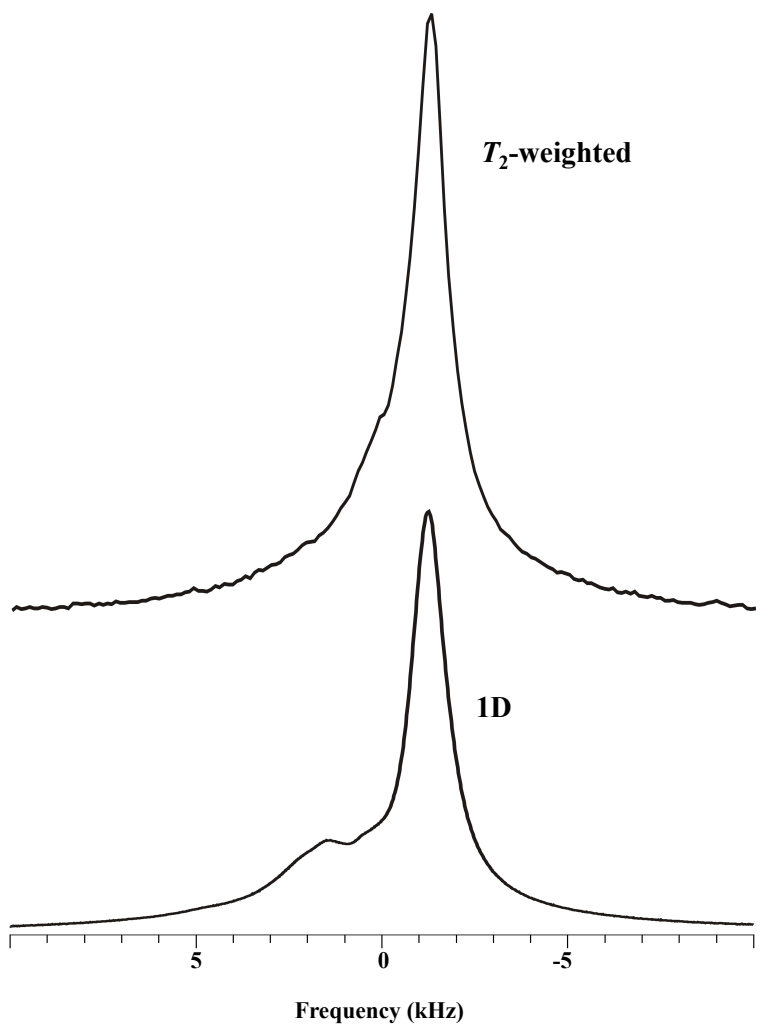

(a)

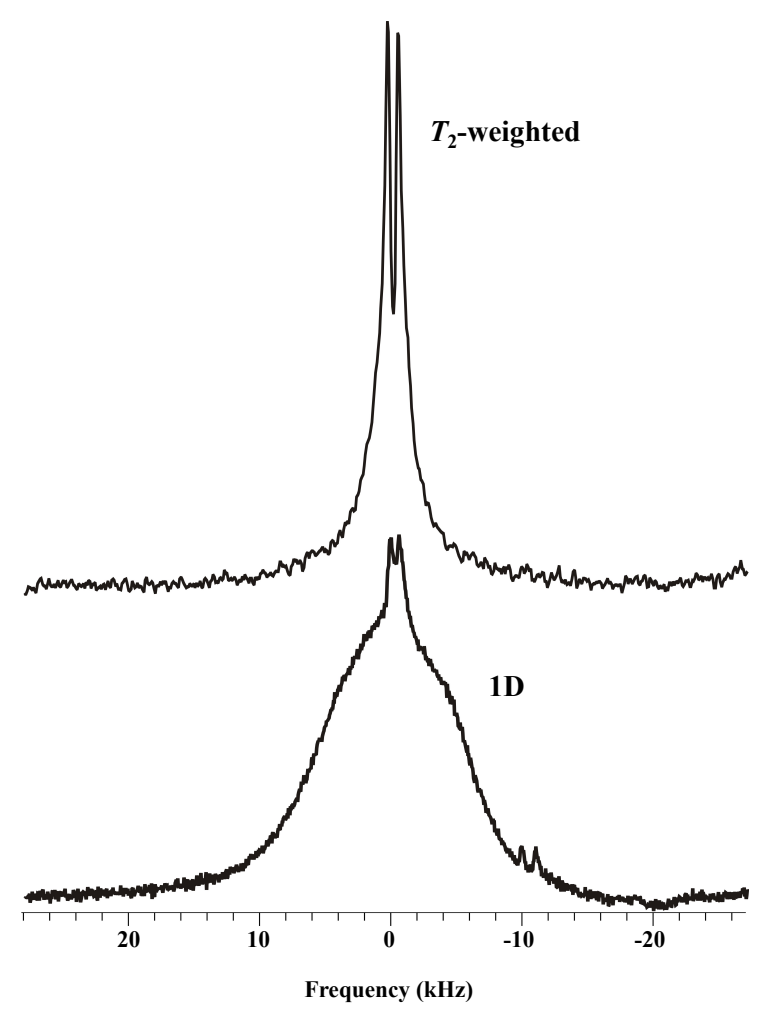

(b)

Figure 3.8: Comparison of static and $T_{2}$-weighted $P F G{ }^{1} \mathrm{H}$ (a) and ${ }^{19} \mathrm{~F}$ (b) spectra, all within phase $\mathrm{S}_{3}$, emphasizing that our PFG-NMR measurements are measuring mobile cations and anions within this material. The $T_{2}$-weighted signals are a result of $T_{2}$ relaxation during the gradient pulse.

We conducted temperature-dependent diffusion experiments between $75-67{ }^{\circ} \mathrm{C}\left(\right.$ phase $\left.\mathrm{S}_{3}\right)$ in $2^{\circ}$ increments to allow measurement of $E_{\mathrm{a}}$ for the cation and anion. Thermal treatment of the material is critical for the following measurements, as $E_{\mathrm{a}}$ is sensitive to small errors or thermally driven changes in $D$. Figure 3.9 is a compilation of all Arrhenius plots with respective $E_{\mathrm{a}}$ values for both cation and anion as a function of thermal treatment. 


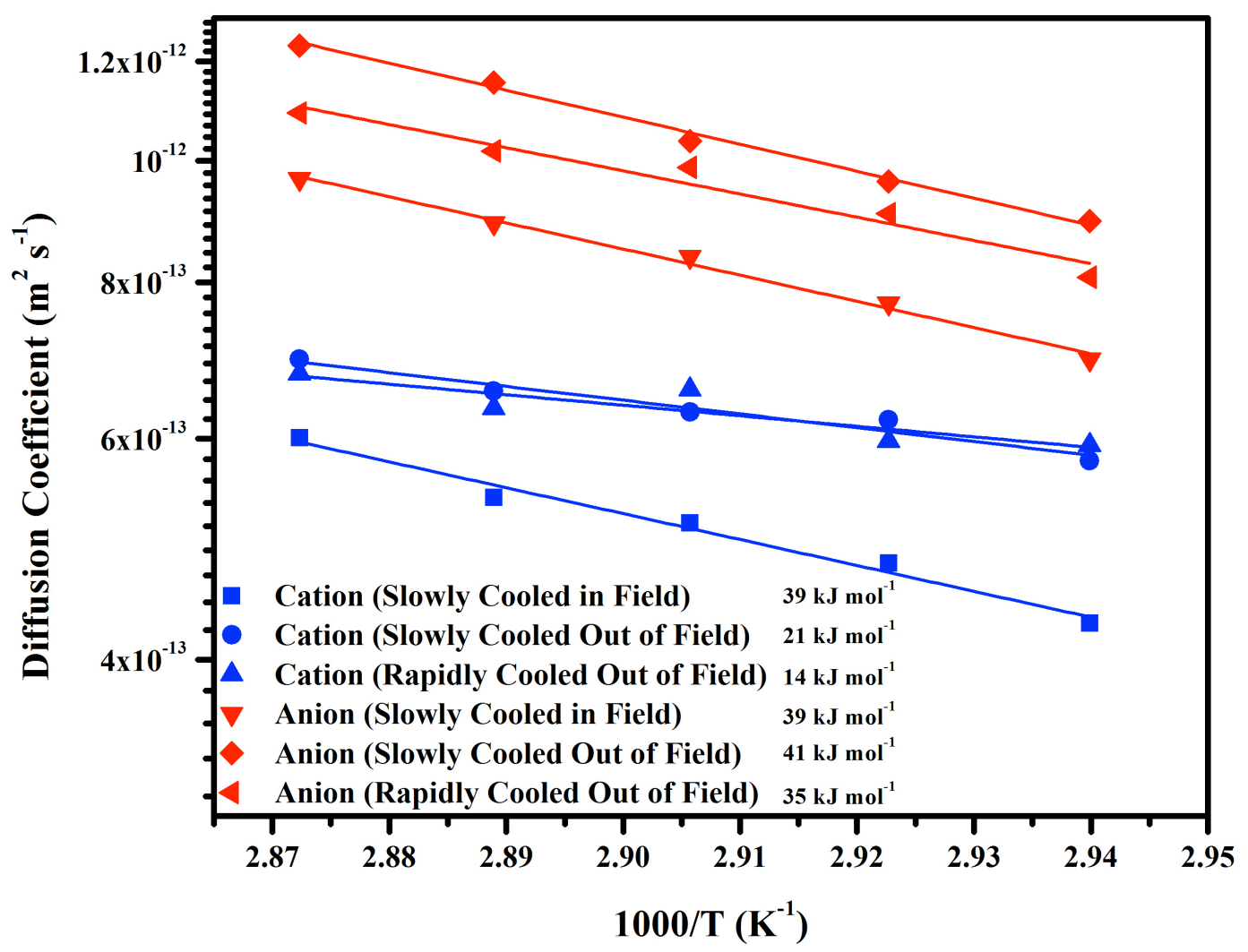

Figure 3.9: Temperature dependence of diffusion coefficient for both cation and anion as a function of thermal treatment and summary of all $E_{\mathrm{a}}$ values extracted from Arrhenius plots. Anion $E_{\mathrm{a}}$ changes fall within error bars as a function of thermal treatment, suggesting that the anion transport mechanism does not change significantly with respect to thermal treatment. Slow cooling in $B_{0}$ causes $E_{\mathrm{a}}$ for the cation to increase drastically to $39 \mathrm{~kJ} \mathrm{~mol}^{-1}$, suggestive of a change in transport mechanism (local molecular environment). The $E_{\mathrm{a}}$ error was calculated to be $+/-5 \mathrm{~kJ} \mathrm{~mol}^{-1}$ using the linest method. While the error is calculated from only one Arrhenius data set (cation fast cooled out of field), we suspect that the error associated with the other measurments are within $+/-1 \mathrm{~kJ} \mathrm{~mol}^{-1}$ of the stated error. 
If we consider both slow cooling thermal treatments (in and out of $B_{0}$ ) and the concepts developed in Chapter 1, slow cooling allows the cations and anions to establish less defective crystallites with fewer and/or smaller grain boundaries are formed. Interestingly, both the cation and anion have $E_{\mathrm{a}}$ values of $39 \mathrm{~kJ} \mathrm{~mol}^{-1}$ in phase $\mathrm{S}_{3}$ when the material is slowly cooled in $B_{0}$. However, when the material is slowly cooled outside of $B_{0}$, cation $E_{\mathrm{a}}$ decreases by a factor of two $\left(39 \mathrm{~kJ} \mathrm{~mol}^{-1}\right.$ to $21 \mathrm{~kJ} \mathrm{~mol}^{-1}$ ) while anion $E_{\mathrm{a}}$ changes fall within error to a range of $35-41 \mathrm{~kJ}$ $\mathrm{mol}^{-1}$, suggesting that the cation is in an environment such that less energy is required for ion transport. Upon rapid cooling out of $B_{0}$, cation and anion $E_{\mathrm{a}}$ changed within error to 14 and $35 \mathrm{~kJ}$ $\mathrm{mol}^{-1}$, respectively, suggesting the local environment does not change significantly depending on whether the sample is slowly or rapidly cooled out of $B_{0}$. Since our $E_{\mathrm{a}}$ values are determined from a $10^{\circ}$ temperature window, we extended this temperature window to $50^{\circ}$ to conclude that our measurements are indeed Arrhenius (not shown).

The cation and anion $E_{\mathrm{a}}$ values in the present study are comparable to that of $\mathrm{H}_{2} \mathrm{O}$ at room temperature $\left(E_{\mathrm{a}}=18 \mathrm{~kJ} \mathrm{~mol}^{-1}\right.$ with a diffusion coefficient on the order of $\left.10^{-9} \mathrm{~m}^{2} \mathrm{~s}^{-1}\right){ }^{21}$ However, the diffusion coefficients for the cation and anion compared to $\mathrm{H}_{2} \mathrm{O}$ differ by three orders of magnitude. While it has been suggested that a viscous liquid in the solid-state exists well below $T_{\mathrm{m}}$, an $E_{\mathrm{a}}$ comparable to that $\mathrm{H}_{2} \mathrm{O}$ does not imply liquid-like dynamics (i.e., isotropic mobility in all directions). While our $E_{\mathrm{a}}$ values suggest liquid-like energetics for the cations and anions on the sub-nm length scale, grain boundaries represent large geometric restrictions; thus it is obvious that changes in environment can change such energetics.

To further rationalize the energetics experienced by the cation and anion we revisit the Arrhenius (Equations 1.2 and 3.2). Contained in the Arrhenius equation is the pre-exponential factor, $D_{0}$, which is defined as diffusion at infinite temperature and contains information about 
the local structure (i.e., the configurational degrees of freedom) of the system governing $E_{\mathrm{a}}$ and $D$ for the diffusing species. We can gain a deeper understanding of the relationship between $E_{\text {a }}$ and $D$ for the cation and anion by comparing their respective $D_{0}$ values. Figure 3.10 shows $D_{0}$ for the cation and anion as a function of thermal treatment.

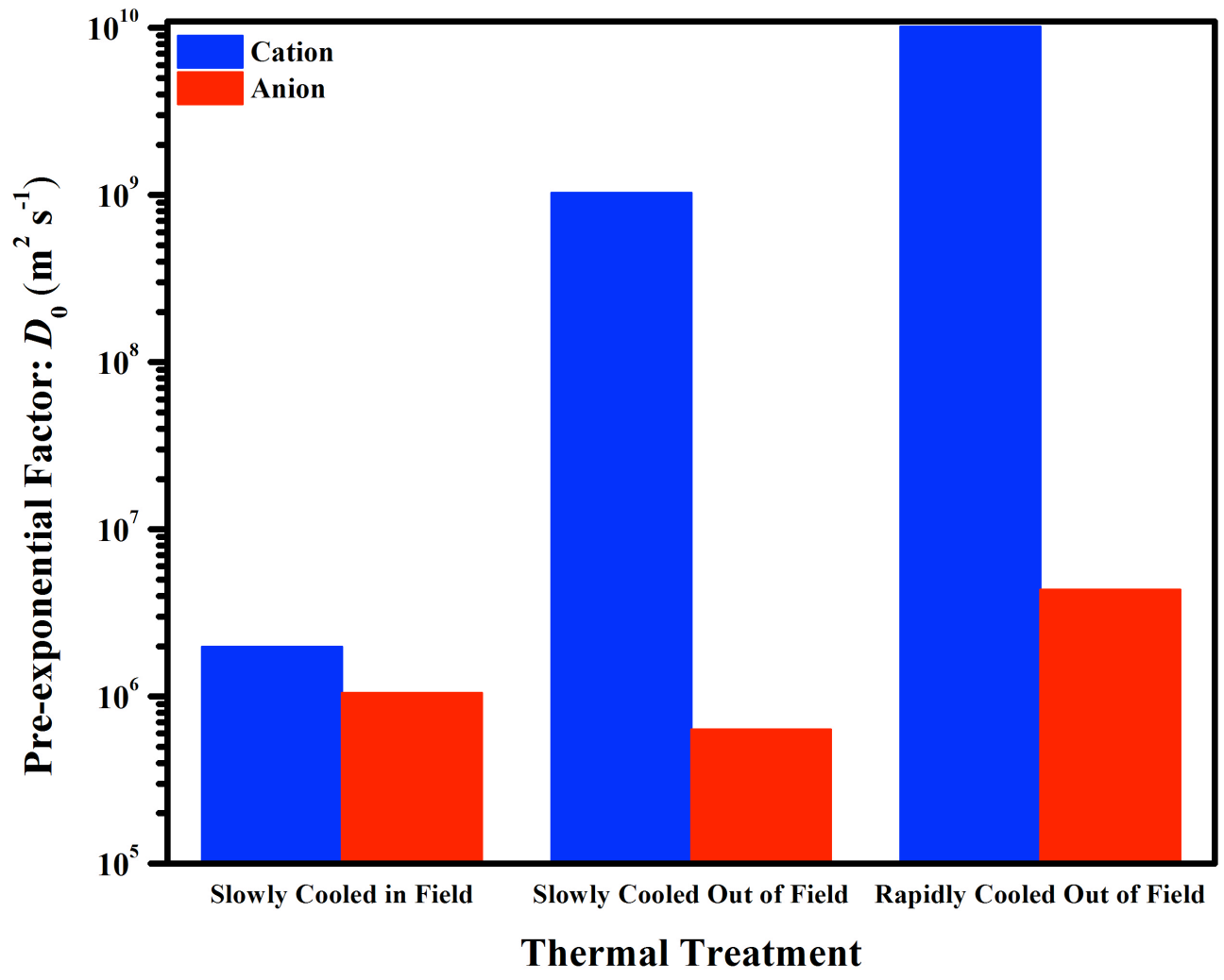

Figure 3.10: Cation and anion $D_{0}$ values as a function of thermal treatment. Cation $D_{0}$ decreases by almost four orders of magnitude from fast cooling out of $B_{0}$ (disordered environment) to slow cooling in $B_{0}$ (more rigid environment). Anion $D_{0}$ changes fall within error bars, suggesting that the configurational degrees of freedom do not change (i.e., the anion transport mechanism does not change with respect to thermal treatment). The $D_{0}$ error was estimated to be $+/-20 \%$. 
It can be seen that $D_{0}$ changes for the anion fall within error bars with respect to thermal treatment, suggesting that the number of degrees of freedom do not change (i.e., the anion local interactions do not change with thermal treatment). Cation $D_{0}$ decreases by almost four orders of magnitude from fast cooling out of $B_{0}$ to slow cooling in $B_{0}$. The slow cooling thermal treatment produces smaller grain boundaries such that the number of degrees of freedom for the cation decreases (i.e., more rigid environment), evidenced by a larger $E_{\mathrm{a}}$ and smaller $D_{0}$ when compared to fast cooling out of $B_{0}$. From our $E_{\mathrm{a}}$ and $D_{0}$ values and residual ${ }^{2} \mathrm{H}$ and ${ }^{19} \mathrm{~F}$ signals, the cation and anion transport in phase $\mathrm{S}_{3}$ most likely occurs via grain boundaries, with conduction dominated by the anion.

We can also take the perspective that essentially an ionic liquid exists in the grain boundary, and thus utilize our experimentally determined $D$ values to evaluate intrinsic properties of the ionic microphase within the grain boundary, such as viscosity $\eta$ and cation/anion hydrodynamic radii $r_{H}$, using the well-known Stokes-Einstein equation (Equation $3.4):^{30,31}$

$$
D=\frac{k T}{6 \pi \eta r_{H}}
$$

$k T$ is the average thermal energy ( $k$ is Boltzmann's constant and $T$ is temperature), $\eta$ is the viscosity of the environment, and $r_{H}$ is the hydrodynamic radius of the diffusing species. As previously reported, $\mathrm{PF}_{6}{ }^{-}$should have $r_{H}=0.25 \mathrm{~nm}$ at $25{ }^{\circ} \mathrm{C} .{ }^{32}$ Knowing this value allows us to calculate a cation $r_{H}=0.34 \mathrm{~nm}$ and a local viscosity $\eta=4.1 \mathrm{~Pa} \cdot \mathrm{s}$; where the latter is similar to viscosities for very viscous ionic liquids. ${ }^{33}$ Since our cation $r_{H}$ and material $\eta$ are calculated from the anion $r_{H}$ (at $25{ }^{\circ} \mathrm{C}$ ) and $D$ values at $45{ }^{\circ} \mathrm{C}$, some error will be associated with our calculations; however, general conclusions can still be drawn. Based upon the Stokes-Einstein 
relation, the measured diffusion coefficients in the present material are a result of cation and anion diffusion of a viscous IL in the grain boundary. It is also possible that variations in cation $E_{\mathrm{a}}$ as a function of thermal treatment are due to changes in ion associations, such as formation of larger/smaller cation/cation, anion/anion, and cation/anion aggregates.

\subsection{Conclusions}

We have presented a new study on the OIPC 1,2-bis[ $N-(N$ '-hexylimidazolium$\left.\left.d_{2}(4,5)\right)\right] \mathrm{C}_{2} \mathrm{H}_{4} 2 \mathrm{PF}_{6}^{-}$by means of multi-nuclear variable temperature SSNMR and PFG-NMR. Not only is this Thesis a new study for the present OIPC, it is a more comprehensive study from an NMR perspective for OIPCs in general, presenting new methods, quantitative data, and definitive insights toward understanding ion transport in such materials. We showed the importance of isotopically labeling these materials with ${ }^{2} \mathrm{H}$ to take advantage of the quadrupolar coupling between $\mathrm{C}^{2} \mathrm{H}$, thus affording spectral observation of changes in local crystallite environment. We propose that it is possible to induce weak crystallite alignment upon slowly cooling in a $9.4 \mathrm{~T}$ magnetic field, which has previously not been reported for any OIPC. Moreover, a change in transport mechanism (local molecular energetics) for the cation occurs in phase $\mathrm{S}_{3}$, evidenced by a change in $E_{\mathrm{a}}\left(14\right.$ to $\left.39 \mathrm{~kJ} \mathrm{~mol}^{-1}\right)$ when slowly cooled in $B_{0}$. $E_{\mathrm{a}}$ as a function of thermal treatment for the anion changes fall within error bars, suggesting that the transport mechanism of the anion does not change. From variable temperature ${ }^{19} \mathrm{~F}$ SSNMR, we also propose that the ion conduction for this OIPC is dominated by anion transport via grain boundaries, and we quantify the fraction of anions in grain boundaries versus those in

crystallites. It is possible that the narrow component observed in our variable temperature ${ }^{19} \mathrm{~F}$ NMR results are a result of isotropically averaged anions within an extended network of defects, 
thus resulting in a "pipe diffusion" mechanism; however, utilization of the Stokes-Einstein equation affords a cation $r_{H}=0.34 \mathrm{~nm}$ and viscosity $\eta=4.1 \mathrm{~Pa} \cdot \mathrm{s}$, suggestive of a viscous IL in the grain boundary. Structurally similar ILs have viscosities within this range. ${ }^{33}$

Further studies on these materials are ongoing, including $E_{\mathrm{a}}$ for the cation and anion within all phases, and the study of materials with different structures. To better understand the ion transport mechanism in each phase, high temperature PFG-NMR should be conducted, and our lab is working toward extending our NMR equipment from a maximum temperature of $80^{\circ} \mathrm{C}$ to $160{ }^{\circ} \mathrm{C}$. DSC measurements similar to those of Rana et al. for the current OIPC might also be interesting, as a $T_{\mathrm{g}}$ may be observed, which would confirm that an amorphous component exists for this material with varying thermal treatment. While these OIPCs do not exhibit remarkable conductivities, doping with small amounts of $\mathrm{LiPF}_{6}$ could potentially enhance conductivity, as previously observed for other OIPCs. ${ }^{1,4,9,17,34-36}$ More in depth ${ }^{2} \mathrm{H}$ NMR studies are also being conducted to better understand the new phenomenon of crystallite realignment upon cooling in $B_{0}$. Electron microscopic studies could potentially help elucidate the nature of the grain boundary and other types of nanometer-to-micron size imperfections present in OIPCs.

We have shown that NMR stands as a very powerful technique toward better understanding of fundamental ion transport mechanisms in soft materials. We will make new advances with NMR and better understand the ion transport mechanisms for not only OIPCs, but also other novel materials; fundamental understanding of such materials is necessary to make advancements and rationally design new materials. 


\section{Acknowledgements}

The authors would like to thank Dr. Minjae Lee and Dr. Harry W. Gibson for providing the OIPC sample and deep discussion, respectively. This work was supported by the National Science Foundation under award number CHE 1057797. Any opinions, findings and conclusions or recommendations expressed in this material are those of the author(s) and do not necessarily reflect the views of the National Science Foundation (NSF). This material is also based upon work supported in part by the U.S. Army Research Office under Grant W911NF-071-0452 Ionic Liquids in Electro-Active Devices (ILEAD) MURI.

\section{References}

(1) Pringle, J. M.; Howlett, P. C.; MacFarlane, D. R.; Forsyth, M. J. Mater. Chem. 2010, 20, 2056.

(2) Timmermans, J. J. Phys. Chem. Solids 1961, 18, 1.

(3) Pringle, J. M.; Shekibi, Y.; MacFarlane, D. R.; Forsyth, M. Electrochim. Acta 2010, 55, 8847.

(4) Howlett, P. C.; Sunarso, J.; Shekibi, Y.; Wasser, E.; Jin, L. Y.; MacFarlane, D. R.; Forsyth, M. Solid State Ionics 2011, 204, 73.

(5) Forsyth, S. A.; Fraser, K. J.; Howlett, P. C.; MacFarlane, D. R.; Forsyth, M. Green Chem. 2006, 8, 256.

(6) Adebahr, J.; Forsyth, M.; MacFarlane, D. R. Electrochim. Acta 2005, 50, 3853.

(7) Adebahr, J.; Grirnsley, M.; Rocher, N. M.; MacFarlane, D. R.; Forsyth, M. Solid State Ionics 2008, 178, 1798.

(8) Adebahr, J.; Johansson, P.; Jacobsson, P.; MacFarlane, D. R.; Forsyth, M. Electrochim. Acta 2003, 48, 2283.

(9) Adebahr, J.; Seeber, A. J.; MacFarlane, D. R.; Forsyth, M. J. Phys. Chem. B 2005, 109, 20087.

(10) Efthimiadis, J.; Forsyth, M.; MacFarlane, D. R. J. Mater. Sci. 2003, 38, 3293.

(11) Efthimiadis, J.; Pas, S. J.; Forsyth, M.; MacFarlane, D. R. Solid State Ionics 2002, $154,279$.

(12) Every, H. A.; Bishop, A. G.; MacFarlane, D. R.; Oradd, G.; Forsyth, M. J. Mater. Chem. 2001, 11, 3031.

(13) Ferry, A.; Edman, L.; Forsyth, M.; MacFarlane, D. R.; Sun, J. Z. Electrochim. Acta 2000, 45, 1237.

(14) Jin, L. Y.; Nairn, K. M.; Forsyth, C. M.; Seeber, A. J.; MacFarlane, D. R.; Howlett, P. C.; Forsyth, M.; Pringle, J. M. J. Am. Chem. Soc. 2012, 134, 9688.

(15) Lee, M.; Choi, U. H.; Wi, S.; Slebodnick, C.; Colby, R. H.; Gibson, H. W. J. Mater. Chem. 2011, 21, 12280. 
(16) Lee, M.; Niu, Z.; Slebodnick, C.; Gibson, H. W. J. Phys. Chem. B 2010, 114, 7312.

(17) Pringle, J. M. Phys. Chem. Chem. Phys. : PCCP 2013, 15, 1339.

(18) Huang, J. H.; Hill, A.; Forsyth, M.; MacFarlane, D.; Hollenkamp, A. Solid State Ionics 2006, 177, 2569.

(19) Long, S.; MacFarlane, D. R.; Forsyth, M. Solid State Ionics 2003, 161, 105.

(20) Pas, S. J.; Huang, J.; Forsyth, M.; MacFarlane, D. R.; Hill, A. J. J. Chem. Phys. 2005, 122 .

(21) Lingwood, M. D.; Zhang, Z.; Kidd, B. E.; McCreary, K. B.; Hou, J.; Madsen, L. A. Chem. Commun. 2013.

(22) Levitt, M. H. Spin Dynamics, 2008; Vol. 2.

(23) Madsen, L. A.; Dingemans, T. J.; Nakata, M.; Samulski, E. T. Phys. Rev. Lett. 2004, 92.

(24) Dingemans, T. J.; Madsen, L. A.; Zafiropoulos, N. A.; Lin, W. B.; Samulski, E. T. Philos T R Soc A 2006, 364, 2681.

(25) Ashbrook, S. E.; Duer, M. J. Concept Magn. Reson. A 2006, 28A, 183.

(26) Li, J.; Wilmsmeyer, K. G.; Madsen, L. A. Macromolecules 2009, 42, 255.

(27) Rana, U. A.; Vijayaraghavan, R.; MacFarlane, D. R.; Forsyth, M. J. Mater. Chem. 2012, 22, 2965.

(28) Dosseh, G.; Fressigne, C.; Fuchs, A. H. J. Phys. Chem. Solids 1992, 53, 203

(29) E. Stejskal, J. T. J. Chem. Phys. 1965, 42.

(30) Edward, J. T. J. Chem. Educ. 1970, 47, 261.

(31) Einstein, A. Annalen der Physik. 1905, 17, 549.

(32) Chen, W. T.; Hsu, W. Y.; Lin, M. Y.; Tai, C. C.; Wang, S. P.; Sun, I. W. J. Chin. Chem. Soc-Taip 2010, 57, 1293.

(33) Yu, G. R.; Zhao, D. C.; Wen, L.; Yang, S. D.; Chen, X. C. AIChE J. 2012, 58, 2885.

(34) Forsyth, M.; Huang, J.; MacFarlane, D. R. J. Mater. Chem. 2000, 10, 2259.

(35) MacFarlane, D. R.; Forsyth, M. Adv. Mater. 2001, 13, 957.

(36) MacFarlane, D. R.; Huang, J. H.; Forsyth, M. Nature 1999, 402, 792. 